\title{
WestVirginiaUniversity
}

THE RESEARCH REPOSITORY @ WVU

Graduate Theses, Dissertations, and Problem Reports

2004

\section{Chaotic price dynamics of agricultural commodities}

Jeff B. Cromwell

West Virginia University

Follow this and additional works at: https://researchrepository.wvu.edu/etd

\section{Recommended Citation}

Cromwell, Jeff B., "Chaotic price dynamics of agricultural commodities" (2004). Graduate Theses, Dissertations, and Problem Reports. 2108.

https://researchrepository.wvu.edu/etd/2108

This Dissertation is protected by copyright and/or related rights. It has been brought to you by the The Research Repository @ WVU with permission from the rights-holder(s). You are free to use this Dissertation in any way that is permitted by the copyright and related rights legislation that applies to your use. For other uses you must obtain permission from the rights-holder(s) directly, unless additional rights are indicated by a Creative Commons license in the record and/ or on the work itself. This Dissertation has been accepted for inclusion in WVU Graduate Theses, Dissertations, and Problem Reports collection by an authorized administrator of The Research Repository @ WVU.

For more information, please contact researchrepository@mail.wvu.edu. 


\title{
Chaotic Price Dynamics of Agricultural Commodities
}

\author{
Jeff B. Cromwell \\ Dissertation submitted to the \\ College of Agriculture, Forestry and Consumer Sciences \\ At West Virginia University \\ In partial fulfillment of the requirements \\ for the degree of \\ Doctor of Philosophy \\ In \\ Natural Resource Economics \\ Gerard E. D'Souza, Ph.D., Chairman \\ Paul Beaumont, Ph.D. \\ Laura Blanciforti, Ph.D. \\ Dale Colyer, Ph.D. \\ Reuben Simoyi, Ph.D. \\ Division of Resource Management
}

Morgantown, West Virginia 


\title{
ABSTRACT \\ Chaotic Price Dynamics of Agricultural Prices
}

\author{
Jeff B. Cromwell
}

Traditionally, commodity prices have been analyzed and modeled in the context of linear generating processes. The purpose of this dissertation is to address the adequacy of this work through examination of the critical assumption of independence in the residual process of linearly specified models. As an alternative, a test procedure is developed and utilized to demonstrate the appropriateness of applying generalized conditional heteroscedastic time series models (GARCH) to agricultural commodity prices. In addition, a distinction is made between testing for independence and testing for chaos in commodity prices. The price series of interest derive from the major international agricultural commodity markets, sampled monthly over the period 1960-1994. The results of the present analysis suggest that for bananas, beef, coffee, soybeans, wool and wheat seasonally adjusted growth rates, ARCH-GARCH models account for some of the non-linear dependence in these commodity price series. As an alternative to the ARCHGARCH models, several neural network models were estimated and in some cases outperformed the ARCH family of models in terms of forecast ability. This further demonstrated the nonlinearity present in these time series. Although, further examination is needed, all prices were found to be non-linearly dependent. It was determined by use of different statistical measures for testing for deterministic chaos that wheat prices may be an example of such behavior. Therefore, their may be something to be gained in terms of short-run forecast accuracy by using semi-parametric modeling approaches as applied to wheat prices. 


\section{TABLE OF CONTENTS}

ABSTRACT. Error! Bookmark not defined.

TABLE OF CONTENTS ...................................................................................................

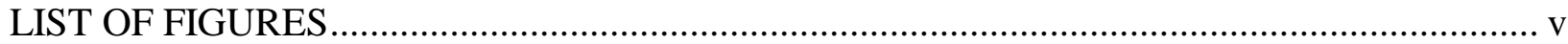

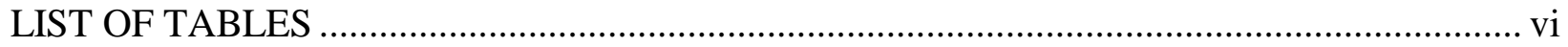

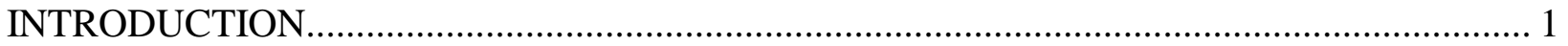

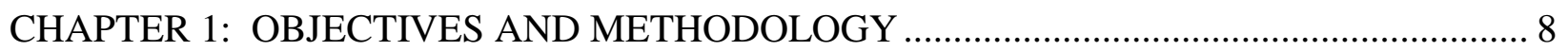

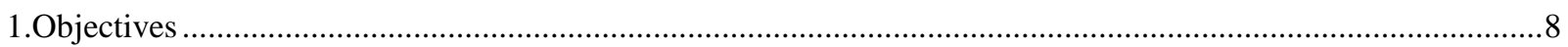

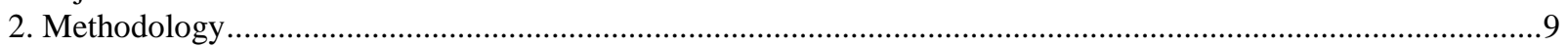

4. Contribution ……………………………………………………………………………………………....

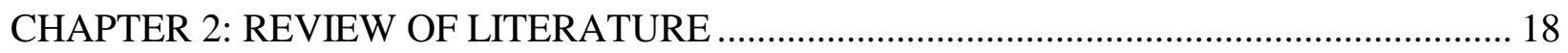

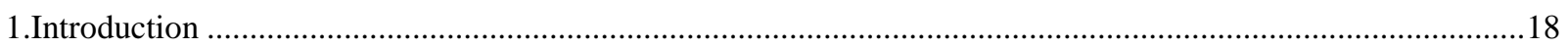

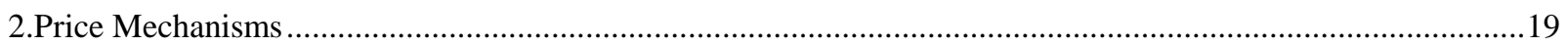

3.Theoretical Modeling of Agricultural Prices ………………………………………………………………...2

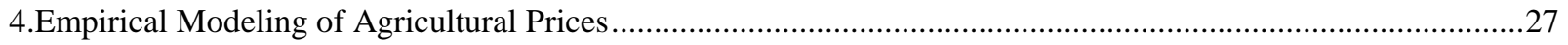

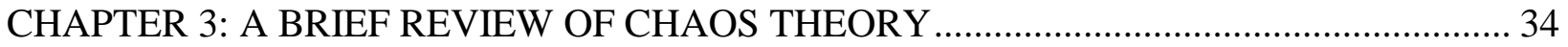

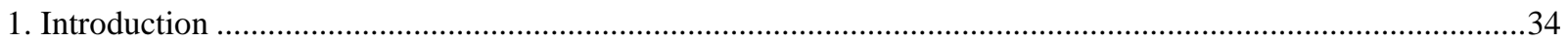

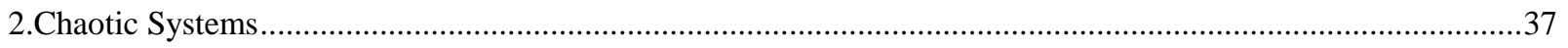

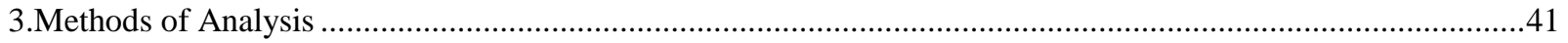

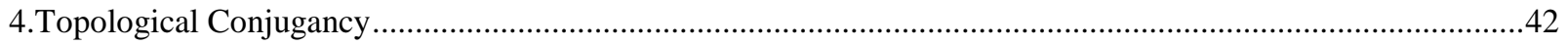

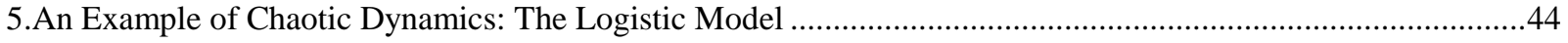

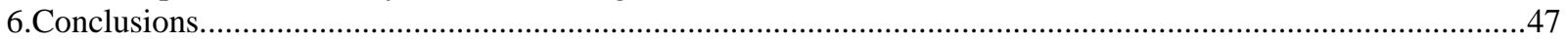

CHAPTER FOUR: AGRICULTURAL MARKET DYNAMICS ............................................. 48

1. Introduction ……………………………………………………………………………………4

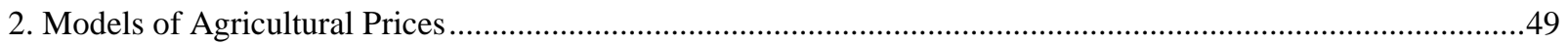

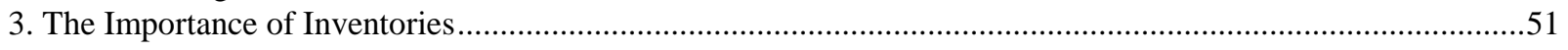

4.Price Dynamics of the Threshold Model ………………………………………………………………...54

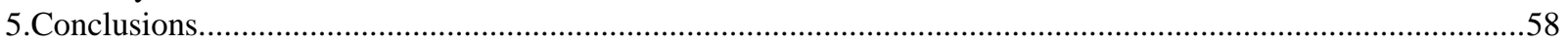

CHAPTER 5: METHODOLOGICAL TESTING FOR DYNAMICAL BEHAVIOR IN

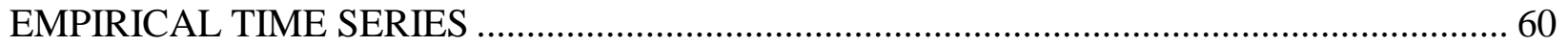

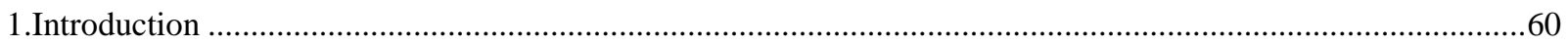

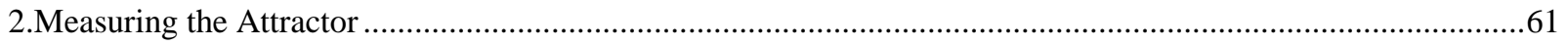

3.Phase Space Construction ………………………………………………………………………………63

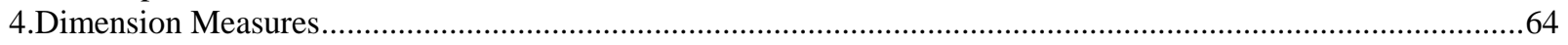

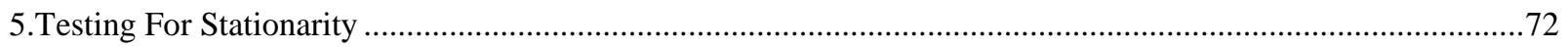

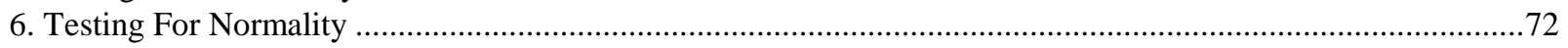

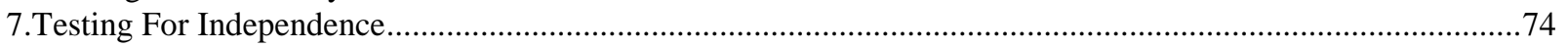

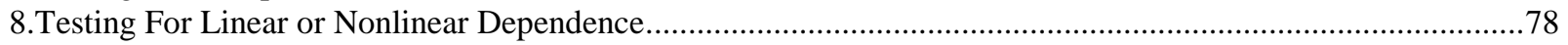

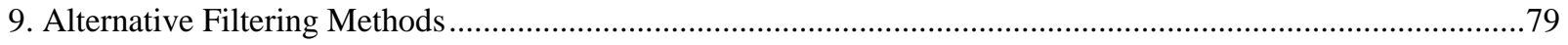

10. Wavelet Analysis ……………………………………………………………………………………8

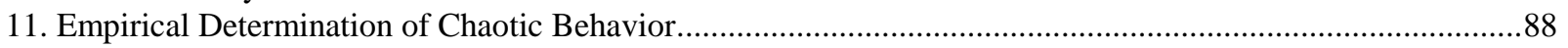

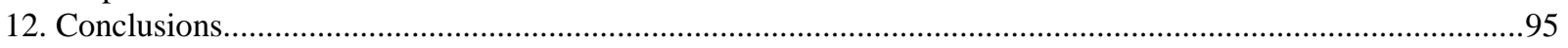

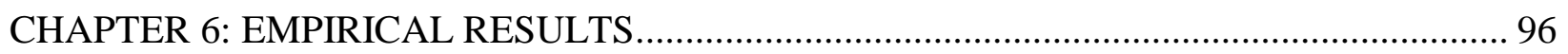

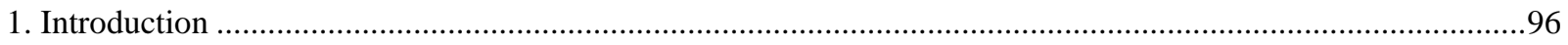

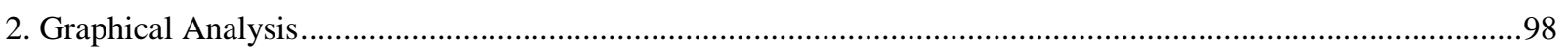

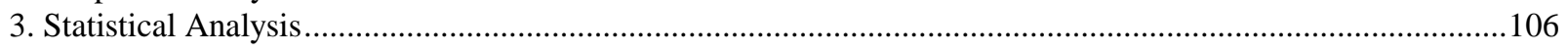




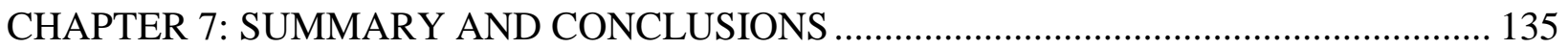

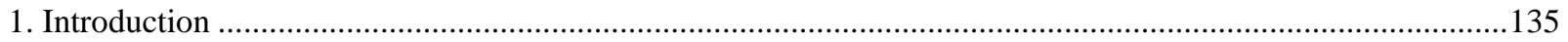

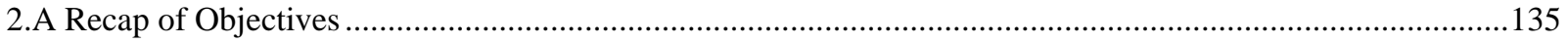

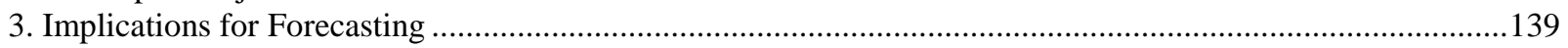

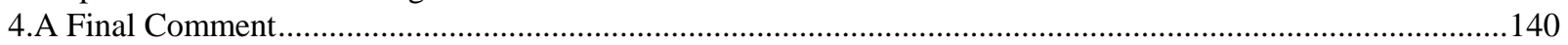

APPENDIX A DATA DESCRIPTION AND SOURCES ............................................... 161

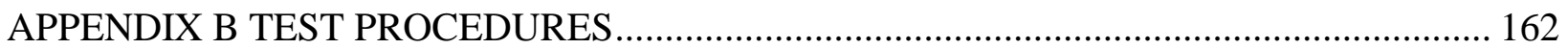




\section{LIST OF FIGURES}

Figure 1.1 Steps for Processing a Univariate Time Series to Test for Independence ........................................12

Figure 1.2 Analytical Tools Used to Determine the Presence of Deterministic Chaos ..........................................16

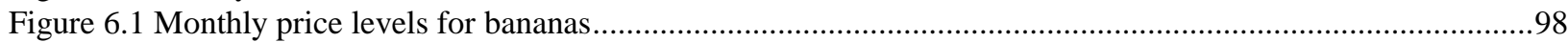

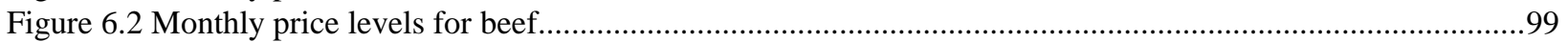

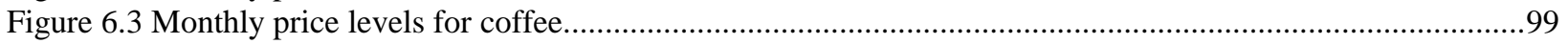

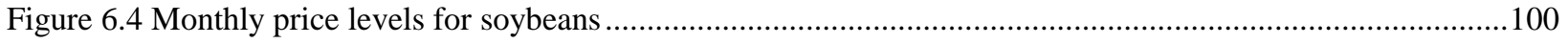

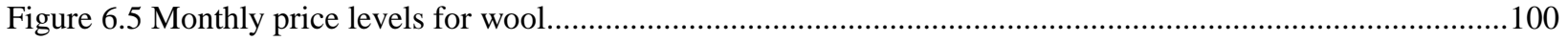

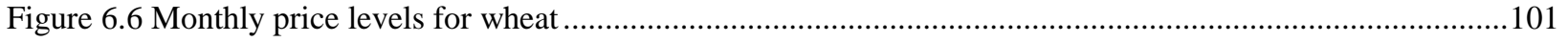

Figure 6.7 Monthly Seasonally Adjusted Growth Rates for Bananas ...........................................................103

Figure 6.8 Monthly Seasonally Adjusted Growth Rates for Beef .............................................................104

Figure 6.9 Monthly Seasonally Adjusted Growth Rates for Coffee.................................................................104

Figure 6.10 Monthly Seasonally Adjusted Growth Rates for Soybeans .............................................................105

Figure 6.11 Monthly Seasonally Adjusted Growth Rates for Wool....................................................................105

Figure 6.12 Monthly Seasonally Adjusted Growth Rates for Wheat ...........................................................106

Figure 6.13 Histogram of Seasonally Adjusted Growth Rates for Bananas....................................................108

Figure 6.14 Histogram of Seasonally Adjusted Growth Rates for Beef........................................................108

Figure 6.15 Histogram of Seasonally Adjusted Growth Rates for Coffee ....................................................109

Figure 6.16 Histogram of Seasonally Adjusted Growth Rates for Wheat......................................................109

Figure 6.17 Power Spectrum of Seasonally Adjusted Growth Rates for Bananas ..........................................113

Figure 6.18 Power Spectrum of Seasonally Adjusted Growth Rates for Beef ...................................................113

Figure 6.19 Power Spectrum of Seasonally Adjusted Growth Rates for Coffee..................................................113

Figure 6.20 Power Spectrum of Seasonally Adjusted Growth Rates for Soybeans ..........................................114

Figure 6.21 Power Spectrum of Seasonally Adjusted Growth Rates for Wool.................................................114

Figure 6.22 Power Spectrum of Seasonally Adjusted Growth Rates for Wheat .................................................114

Figure 6.23 Wheat Spectral Components for the Sampling Rate of $1 / 2$ to 1 Month..........................................116

Figure 6.24 Wheat Spectral Components for the Sampling Rate of 1/4 to 1/2 Months .......................................117

Figure 6.25 Wheat Spectral Components for the Sampling Rate of 1/6 to 1/4 Months .......................................117

Figure 6.27 Wheat Spectral Components for the Sampling Rate of 1/10 to 1/8 Months .......................................118

Figure 6.28 Wheat Spectral Components for the Sampling Rate of 1/12 to 1/10 Months .......................................119.

Figure 6.29 Wheat Spectral Components for the Sampling Rate of $1 / 14$ to $1 / 12$ Months ......................................119

Figure 6.30 Wheat Spectral Components for the Sampling Rate of 1/16 to 1/14Months ...................................120 


\section{LIST OF TABLES}

Table 1.1 Agricultural Commodity Prices' Stylized Facts, 1960.01-1994.06 .......................................................5

Table 1.2 A Correlation Matrix for Agricultural Price Growth Rates, 1960.02-1994.06 .......................................5

Table 3.1 Summary of Characteristics Associated with Equation (3.3) -the Logistic function , $\mathrm{x}_{\mathrm{t}+1}=\mu \mathrm{x}_{\mathrm{t}}\left(1-\mathrm{x}_{\mathrm{t}}\right) \ldots \ldots \ldots . . .46$

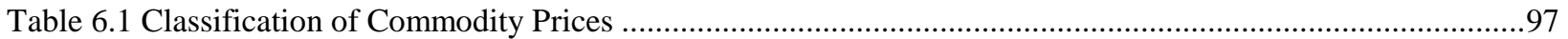

Table 6.2 Summary Statistics of Seasonally Adjusted Growth Rates (1960.02-1994.06) ...................................107

Table 6.3 Tests for Stationarity on Seasonally Adjusted Prices .............................................................112

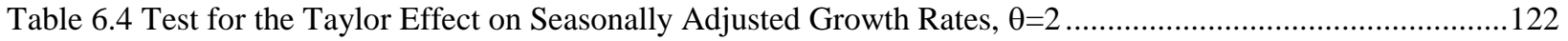

Table 6.5 Residual Diagnostics for Linear Models of Seasonally Adjusted Growth Rates .................................124

Table 6.6 Third Moment Test for Linearly Filtered Residuals ..........................................................................126

Table 6.7 GARCH $(1,1)$ and ARCH $(3)$ Model Estimates for the Conditional Variance .......................................127

Table 6.8Residual Diagnostics from ARCH and GARCH Models.................................................................129.

Table 6.9 The Root Mean Square Error Forecast Statistics for AR, ARCH-GARCH, Neural Network and Nearest

Neighbor Models For Forecast From June, 1993 To June 1994 .....................................................130

Table 6.10 Correlation Dimension and Largest Lyapunov Exponent Estimation Results for Wheat Growth Rates..132

Table 6.11 Kolmogorov Entropy Estimates for Wheat Price Growth Rates.... 


\section{INTRODUCTION}

The discovery of chaos has created a new paradigm in scientific modeling. On the one hand, it implies new fundamental limits on the ability to make predictions. On the other hand, the determinism inherent in chaos implies that many random phenomena are more predictable than had been thought. -James Crutchfield

As we close this millennium, we have begun to re-examine old questions within the framework of a new methodology. Two new theories, one known as Complexity theory and the other Chaos theory seek to uncover the geometrical structures of systems as they go through their associated state transitions. Complexity theory examines the emergence of order or structure and chaos in systems that involve numerous adaptive subsystems which feedback in positive and/or negative ways. Chaos theory attempts to describe the behavior of these complex systems. At the heart of both of these theories is the question of prediction. The foundation of all science is based upon the predictive model and this is not different with respect to economic science. As is discussed later in this work, a fundamental characterization of chaotic systems is that they become unpredictable at an exponential rate. This unpredictability can have important implications for economic policies at both the micro and macro levels.

For example, historically, the prices of internationally traded agricultural commodities have demonstrated considerable leverage in the amplitude of farm incomes, consumer welfare, and the quantity of export earnings for many countries. Tomek and Robinson (1990) contend that nearly fifty percent of the world's population income is principally determined by the prices received for agricultural commodities. Therefore, predicting the temporal behavior of internationally traded agricultural commodity prices is of great importance particularly to the 
less-developed countries that depend heavily on export earnings from these commodities. Because of this dependency between export earnings and GDP, the economies as well as the macroeconomic and microeconomic policies of these countries are likely to be adversely affected by a decline in the price of international traded agricultural commodities. For example, a drop in price of only a few cents per pound can have an ardent political and economic backlash in such countries as Mauritius, Colombia, and Ghana. Furthermore, even the United States is not immune to price variability as one could confirm from recent various news sources. Tomek and Robinson (1990) recount that in the mid-seventies there were the consumer boycotts of meat purchases and the ensuing tractor marches on Washington, D.C. Because of the political and economic implications of undesirable temporal changes in agricultural price behavior, it is necessary to examine some of the empirical characteristics of internationally traded agricultural commodity prices.

One fundamental feature of internationally traded agricultural commodity markets as opposed to nonfarm goods and services, concerns their price volatility. Historically, these prices are found predisposed to be extremely volatile. The international price of sugar is a stylized example of volatility. ${ }^{1}$ During a twelve-month period in the early eighties, the international price of sugar fell seventy percent and deteriorated further in succeeding years until rebounding threefold. Table 1.1 shows a set of stylized facts for internationally traded bananas, beef, coffee, soybeans, wheat and wool prices over a three-decade period from 1960 to $1990 .^{2}$ The coefficient of variation for each empirical price series provides information on the degree of volatility

${ }^{1}$ Labys et al. (1991) has suggested five basic characteristics of aggregate food markets; inelastic demand, slow growth in total demand, competitive market structure, significant technological change, and the tendency of resources to become fixed within the agricultural sector.

2 These are the prices used throughout the subsequent chapters. Data sources are in Appendix B. 
unveiled by the empirical price series. Unquestionably, the decade of the seventies was an inordinately turbulent time for agricultural commodity prices. One can use the two inflationary periods present in the seventies as one possible explanation. For example, for wheat prices the coefficient of variation increased from five percent in the decade of the sixties to almost fortypercent in the seventies. In the decade of the eighties, notice that wheat volatility decreased from it is high in the seventies but did not revert to the sixties levels.

As discussed further in chapter 4, fluctuations in agricultural prices tend to occur because of disturbances in supply conditions. As an example, consider the annual supply from tree crops that cannot be easily adjusted since current output is heavily dependent upon the stock of trees. Thus, wide and lengthy price swings may result from several attempts to adjust this stock. In the medium run, the supply from annual crops may vary depending on the situation between the period of planting and of harvest. If a farmer's expectation of next year's demand is in error, price fluctuations result from over and under supply. In the very short run, factors such as weather during the growing or harvest season often cause price fluctuations. ${ }^{3}$ Palaskas and Varangis (1991) state that the most plausible explanation for the long run volatility of commodity prices during the seventies and eighties has to do with the effect of macroeconomic variables, such as interest rates, exchange rates, and income growth in industrial countries.

Since prices of internationally traded agricultural commodities are extremely unstable, an understanding of the stochastic processes governing these price movements is essential for macroeconomic management, for national consumption and saving policies, for agricultural pricing policies, and for the design of risk sharing mechanisms between farmers, resource

\footnotetext{
${ }^{3}$ Weather changes play an important role in the ability of farmers to plan for future contingencies. In later chapters, the term noise is to be used to refer to the inability to quantify this factor.
} 
holders, and government. ${ }^{4}$ Increasingly, agricultural economists are recognizing the importance of assessing uncertainty in forecasting and of using this information in a decision-making framework. Currently, the accurate forecasting of agricultural, as well as other commodity prices and quantities remains a formidable problem. The economic structure underlying commodity markets can change more rapidly than that of national economies. Particularly in the last decade, inflation, devaluation, and several petroleum crises have caused the underlying structures to change in a way that has adversely influenced the forecasting capabilities of corresponding models.

Because of the empirical irregularity of internationally traded agricultural prices, they have often been cited as examples of randomness. Common convention prescribes randomness to be viewed as a class of processes that are uncertain or unpredictable. ${ }^{5}$ This implies that the behavior of an economic system with respect to some outcome variable is fundamentally stochastic or unpredictable. ${ }^{6}$ Therefore, economic processes have both structural and random components. Specifically, Liu, Granger and Heller (1992) state that the choice is to view economic processes as

- Linear in structure with insignificant stochastic elements, or

- Nonlinear structure with insignificant stochastic forces, or

- Nonlinear structure but with significant stochastic shocks

4 Gilbert (1990) illustrates this concern by pointing out that the International Commodities Division of the World Bank is required to construct price forecasts for all major agricultural commodities over a long-term horizon on an annual basis.

5 The mathematician Persi Diaconis has suggested that the more we contemplate randomness the less random things appear (in Albers and Alexanderson, 1985).

${ }^{6}$ Alternatively, predictability is what enables us to determine an unknown state of a system from a known state. This is accomplished by applying the known state as input to a predictive algorithm. 
Table 1.1 Agricultural Commodity Prices' Stylized Facts, 1960.01-1994.06

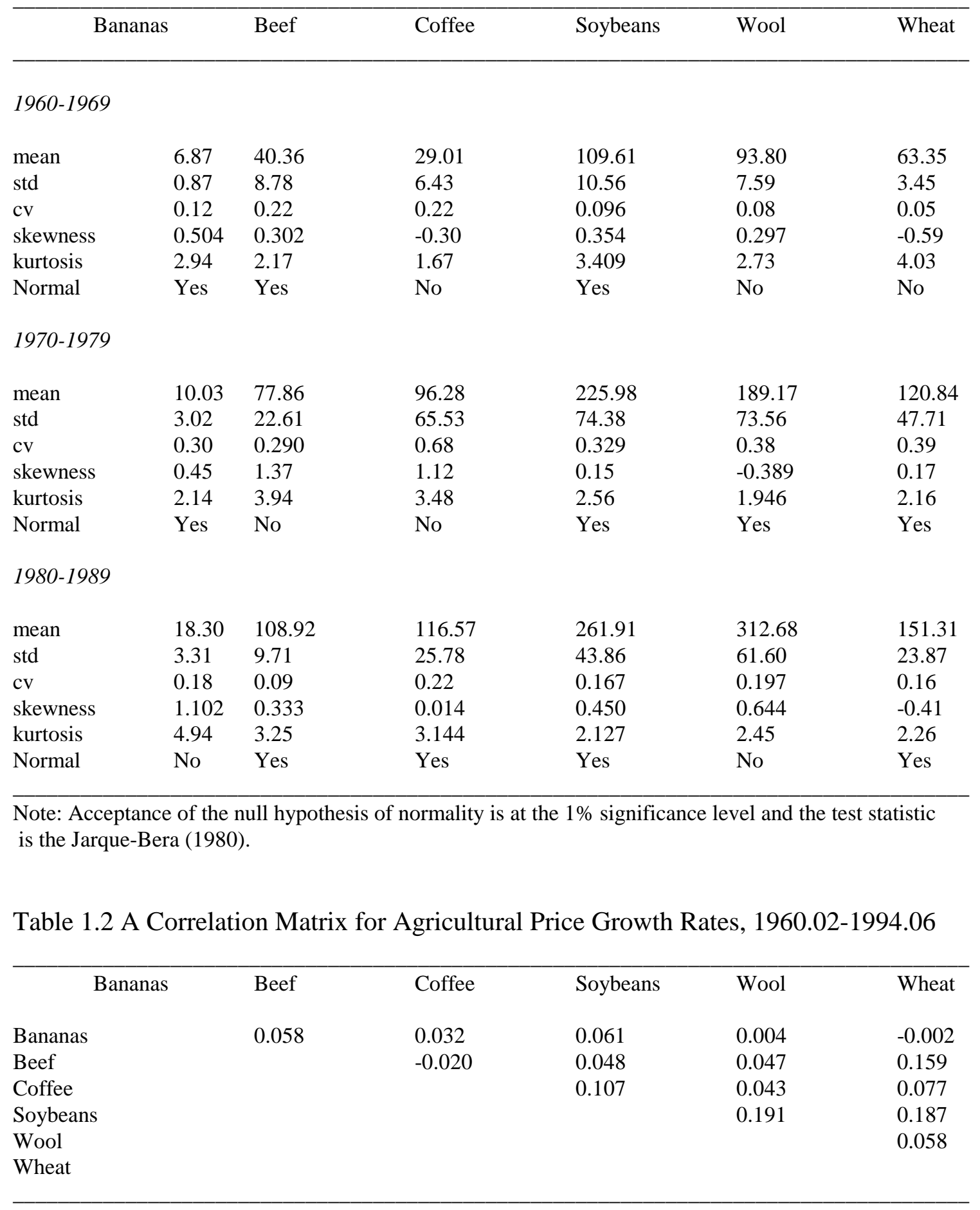


Furthermore, gathering more information simply does not eliminate the randomness or the predictability. Crutchfield (1986) writes:

In principle, the future is completely determined by the past, but in practice, small uncertainties are amplified, so that although the behavior is predictable in the short run, it is unpredictable in the long run. There is order in chaos: underlying chaotic behavior there are elegant geometric forms that create randomness in the same way as a card dealer shuffles a deck of cards or a blender mixes a cake batter.

As an alternative, to a stochastic or quantum system, we can approach the problem of prediction from the perspective of Scientific Determinism made popular by Pierre-Simon de LaPlace. This traditional approach in economics and other social sciences is to make a prediction of a system based on the mathematical representation (i.e., an algorithm, which could also be verbal) of an economic theory upon which extrapolation of past behavior of the variable inputs generates a future description of important economic variables. The implicit assumption is that there exists a "closed form" representation of the system that generates an "accurate" forecast of the system. However, the case may be that this is an erroneous assumption. ${ }^{7}$

The field of chaos theory has demonstrated the ability to have mathematical representations (i.e. closed forms) that generate unpredictability. Thus, chaos represents a gray area between randomness and determinism. In other words, it is appropriate to acknowledge the unpredictability of chaotic systems and yet broaden our knowledge of scientific explanation. The one promise of chaos theory is that simple deterministic models can offer a more accurate forecasting model for commodity prices. This is similar to weather forecasting where an accurate forecast over a period of a couple of days is taken seriously but not one over a month. However, seasonal cycles do play an important role in these monthly forecasts.

${ }^{7}$ Clearly, this is the case in some examples generated in number theory. 
In order to state the problem of this work, it is important to define what a predictable system is. Stone (1989) states the criteria for a predictable system:

- An algorithm that maps a state of the system at any given time to a state at any other time, and the algorithm is not probabilistic;

- A system that a given state is always followed by the same history of transitions;

- Any state of the system can be described with an arbitrarily small (nonzero) error;

- Any state of the system can be generated from the algorithm with an arbitrarily small (nonzero) error from any other state of the system. ${ }^{8}$

The first and second assumptions as outlined above state that the algorithm of the system is the mean of the process, which is assumed constant, i.e., stationarity conditions. The last two have to do with the assumption invoked in this work of choosing an appropriate loss function, i.e., mean square error. Throughout the rest of this work, our task is to uncover the dynamics of agricultural markets and investigate the properties that lie therein.

${ }^{8}$ Chaotic systems satisfy the first three propositions. 


\section{CHAPTER 1: OBJECTIVES AND METHODOLOGY}

The purpose of this study then is to determine if the empirical representations of internationally traded, agricultural commodity prices demonstrate random or pseudo-random behavior. Such pseudo-random behavior is a characteristic of a deterministic chaotic process. ${ }^{9}$

\section{Objectives}

To find a solution to the posed problem, the attainment of several objectives is sought. They are:

1. To provide a review of current as well as past attempts to model agricultural prices, most notably, commodity prices. This review is to not only consist of past attempts at modeling agricultural prices but also to contrast them with the basic tenets of chaos theory.

Objective 1 is presented to initiate the reader into the historical context of the work. In addition, a cursory overview of chaos theory provides the reader with a solid foundation with which to view the assumptions and implications of the work presented here.

2. To demonstrate on a theoretical level the necessary conditions for an agricultural market to exhibit chaotic behavior. This is to be completed by formulating a competitive market model for agricultural commodities and then determining any necessary restrictions to be enforced on the parameters and functional forms of the model.

\footnotetext{
${ }^{9}$ Deterministic chaos as used here refers to the parametric structure (i.e., closed form representation) for a system that generates apparent random behavior of the future (Clearly, this the case in some examples generated in Number theory. A more detailed definition and explanation are given in Chapter 3.) Alternatively, predictability is what enables us to determine an unknown state of a system from a known state that is done by applying the known state as input to a predictive algorithm.
} 
Objective 2 is formulated to provide a substantial theoretical foundation for the existence of chaos in commodity prices. Quite often, empirical studies become exercises or demonstrations of the latest statistical and mathematical tools. This is not the purpose here.

3. To examine the current methodology for the procedures used in the detection of chaotic behavior. Also, to suggest possible improvements where necessary and develop the testing framework into a logical and coherent one.

Objective 3 is provided to insure that a rigorous analysis is presented. The rigor stems from the ability of the testing methodology commonly used in the chaos literature.

4. Using the testing framework developed under objective three, to test for the presence of chaotic behavior in a sample of internationally traded agricultural commodity prices. The sampling interval for these prices is monthly and was obtained over the period 19601994. The list of prices includes bananas, beef, coffee, soybeans, wool, and wheat. ${ }^{10}$

Burton (1993) has stated that, with the exception of the Chavas and Holt (1991) study, there has been relatively little empirical testing of economic time series for the presence of chaos, particularly in the field of agricultural economics. Objective 4 is the main emphasis of this work, intended to provide an empirical classification scheme for forecasters of internationally traded agricultural prices. While the attainment of Objective 2 enables forecasters to postulate the probability of the market being chaotic, it, however, it in no way requires it to be. This objective provides empirical evidence to further strengthen or weaken the chaotic assumption.

\section{Methodology}

To satisfy the objectives presented, the method of approach involves three stages. These evaluative stages are: (1) A review of agricultural price dynamics and chaos theory (OBJECTIVE 1); (2) chaotic behavior in an agricultural commodity market (OBJECTIVE 2); (3) An

10 Sources for these prices are in Appendix A. 
examination of the testing framework (OBJECTIVE 3); and (4) empirical testing for chaos (OBJECTIVE 4).

The first stage involves a review of agricultural commodity price behavior. Commodity price behavior is to be examined from both the short and long run perspectives, including influential factors, as well as the role that inventories and expectations play in the formation of agricultural price behavior. A review of empirical studies of price behavior is also included to examine concepts such as martingales, modeling conditional means and variances, and linear and nonlinear approaches to modeling the dynamics of agricultural commodity markets.

Next, a brief and cursory review of the basics of chaos theory is presented. Appropriate definitions and properties of chaotic systems are presented along with the techniques of analysis for these systems. An example of a chaotic system is presented to ease the reader's transition from the linear paradigm and it has associated cobwebian dynamics to the nonlinear paradigm and chaos theory. ${ }^{11}$

In stage 2, an agricultural commodity market model is proposed and its dynamics assessed. The analysis of the market dynamics relies on the property of topological conjugacy for ascertaining the periodic orbit structure, counting the periodic points, and establishing the existence of aperiodic points for the proposed model. The linkage between the proposed model and the current literature is established. Critical assumptions about alternative models are briefly addressed.

The third stage of the analysis involves empirical testing as defined in Figure 1.1. For the sake of clarity, two secondary stages have been identified. The first stage is an attempt to 
decipher the nature of the linear as well as nonlinear dependence in selected commodity price series. This procedure involves tests for stationarity, normality, independence and nonlinear dependence. In the first step, the series is seasonally adjusted, and where necessary transformed from a non-stationary series to become covariance stationary. The degree of differencing is determined from standard tests of the unit root hypothesis, i.e., the Dickey-Fuller (1979) tests. Since our concern is principally with the nature of dependence in commodity prices, the second step involves testing for normality to use the mathematical relations between stationarity, normality and dependence. If a time series possesses all of the latter properties, then it is linearly dependent. However, if a series is stationary, but non-normal and dependent, then either a linear or a nonlinear model could be the correct specification.

11 The reference here to cobwebian dynamics relates to the adherence of economists to the linear paradigm of convergent and divergent behavior. The cobweb model popularized by Ezkeil (1938) is to demonstrate the concept of equilibrium behavior as an outgrowth of convergence. 
Figure 1.1 Steps for Processing a Univariate Time Series to Test for Independence

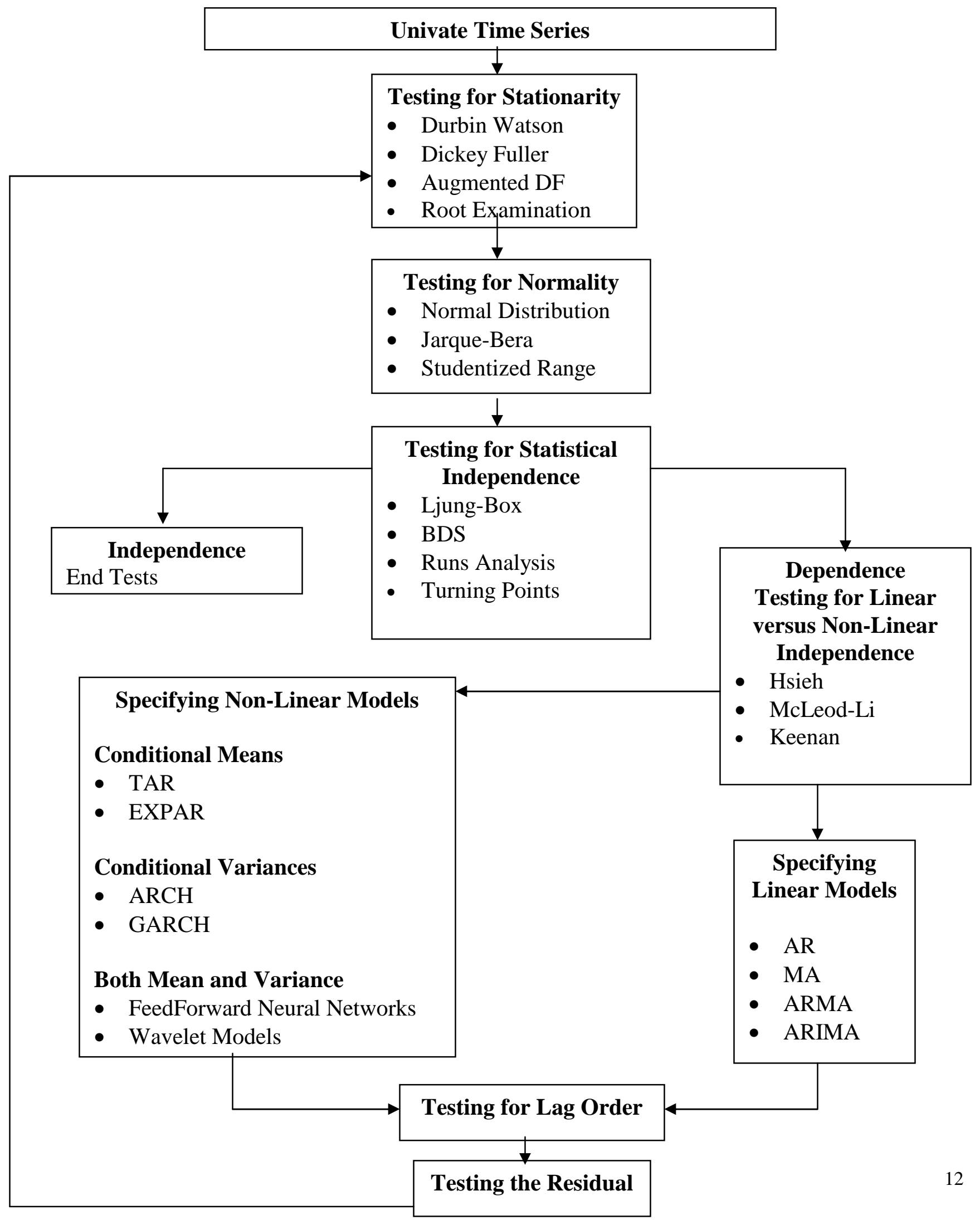


The third step involves advancing to tests of the property of independence itself. The main test employed is that of Brock, Dechert and Scheinkman (1986). As stated previously, the testing process is greatly simplified when the time series is stationary and normal. However, when the series is non-normal (often a case for commodity prices expressed in growth rates), a procedure must be adopted which can discriminate between linear and nonlinear dependence. For example, if the data are pre-filtered by fitting a linear structure to the conditional mean (i.e. using a linear autoregressive model, $\operatorname{AR}(p))$, then the residuals from these $\operatorname{AR}(p)$ models can be examined for independence. If the residuals are not independent, then we may conclude that a nonlinear structure exists. In this case, the normal approach is to use the BDS test based on an iterative process of linear and/or nonlinear filtering until the null hypothesis of independence in the residuals is accepted.

If the null hypothesis is rejected, then the fourth step requires that we test for nonlinearity. This typically involves using the (third moment) test of Hsieh (1989), which discriminates between additive and multiplicative forms of nonlinearity. Examples of models that exhibit additive nonlinearity are the bilinear, threshold and exponential autoregressive specifications. Alternatively, multiplicative nonlinearity is demonstrated in the autoregressive conditional heteroscedastic model (ARCH) of Engle (1982) and the generalized autoregressive heteroscedastic model (GARCH) of Bollerslev (1986). An investigative model that considers additive as well as multiplicative types of nonlinearity is the autoregressive conditional heteroscedasticty in the mean (ARCH-M) model used by Diebold and Nerlove (1988). If one finds multiplicative nonlinearity, $\mathrm{ARCH}$ or the parsimonious GARCH models can be fitted to the 
data, and the BDS test can be used to examine the standardized residuals for independence. Discovering the presence of nonlinearity has recently led to further testing for chaotic behavior.

Because of the emphasis on parameterization of the above model fitting procedures, a semi-parametric approach is presented in the form of feedforward neural network models (Heicht and Nielson, 1990) and White (1992). Furthermore, we can decompose the time series into the frequency domain and does further non-parametric work by the use of wavelet analysis. This adds a degree of robustness to the results and helps in noise reduction and outlier determination (Greenblatt, 1994). These models have the ability to include the ARCH family of models as well as other linear and nonlinear specifications. The ability of these models to generalize has generated considerable interest in using them as a standard test of model specification (Lee et al., 1993.) as well as prediction.

The procedure for testing, as a part of the second stage of the testing framework for chaos , extends the above tests for nonlinear dependence. The requirements most often stated for a time series to be chaotic are that it must satisfy the following two conditions:

- A nonlinear model must generate the series. ${ }^{12}$

- The solution to that model must be sensitive to initial conditions.

This demands that one first search for a possible nonlinear specification by estimating the correlation exponent or dimension; this exponent detects the amount of correlation that exists in higher dimensions. One then tests for sensitive dependence by measuring the rate of divergence of the long-term trajectory (given insignificant differences in initial conditions) using

12 Non-linear as used here refers to non-linear in the variables as opposed to non-linear in the parameters. 
the Lyapunov exponent (Wolf et al., 1985). Only one positive Lyapunov exponent value is sufficient to describe the system as chaotic, while negative exponents indicate stability.

After a linear model is used to remove the dependence from the time series; tests based on the above two measures can be conducted on the residuals. Utilizing the residuals in analyzing chaotic behavior of data originated in Brock (1986) and has been utilized in studies by Brock and Dechert (1986), Brock et al. (1991), Brock and Baek (1991) and Sayers (1990).

In the next stage of the study, the results of the tests as applied to the prices of agricultural commodities exchanged on the major international commodity markets are generated. The prices sampled monthly over the period 1960-1994, are for bananas, beef, coffee, soybeans and wheat. The areas tested include the results of stationarity testing, linear specification, residual diagnostics I, nonlinear model specification, residual diagnostics II, and chaos testing.

Chaos testing methodology is presented in Figure 1.2. From both the subjective and more objective approaches, we can use the procedure of graphical inspection as well as obtaining estimates as a result of dimensional analysis. Dimension analysis includes obtaining characteristic roots, and their associated rates of change to detect the presence of deterministic chaos. 
Figure 1.2 Analytical Tools Used to Determine the Presence of Deterministic Chaos

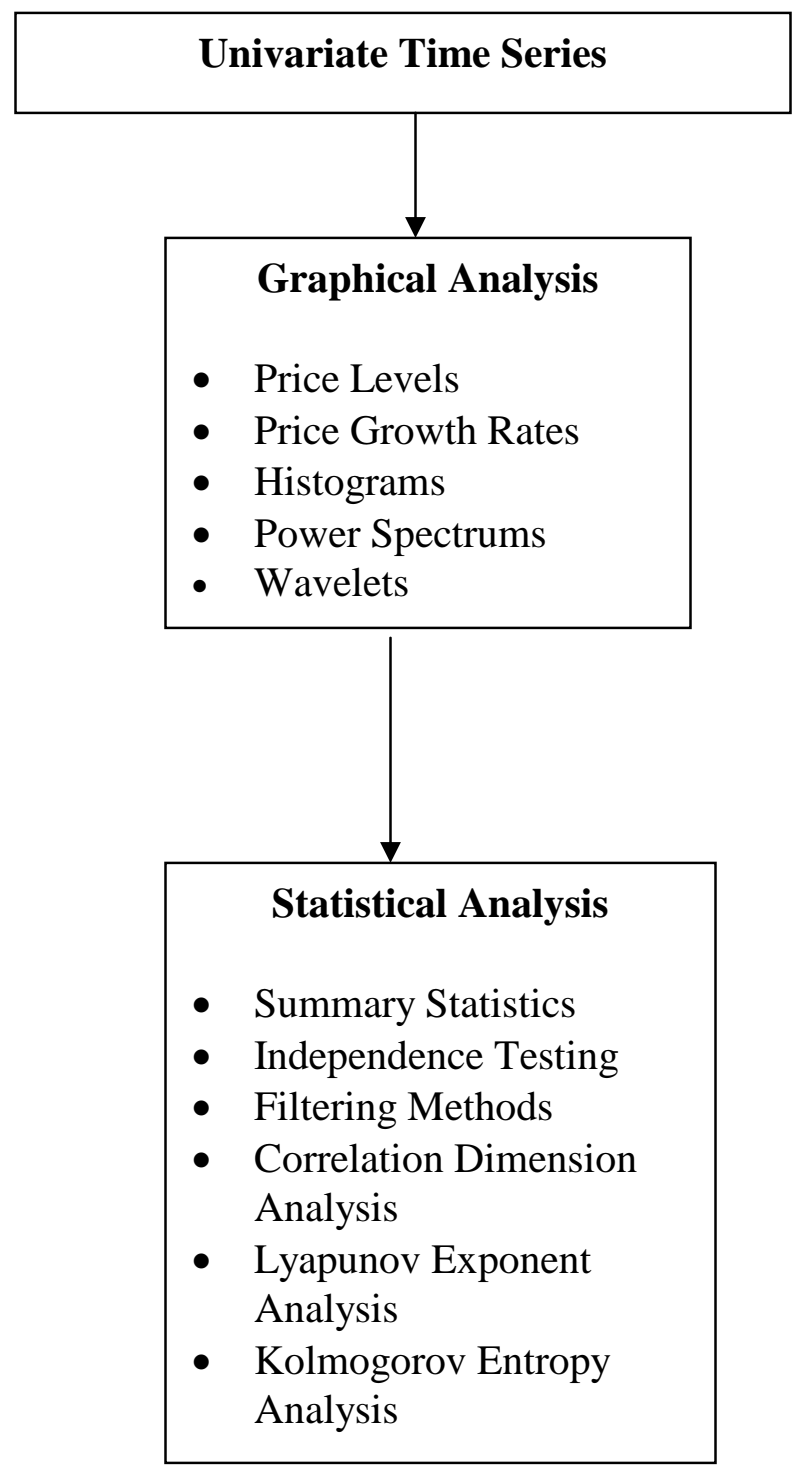

\section{Contribution}

Once the objectives of the study are achieved, several contributions can be identified. The first contribution involves the methodology. Only a handful of studies have examined chaos testing in a commodity market. Of those studies, this is the first to develop a theoretical market 
model, obtain its reduced form, and examine its dynamics from a theoretical perspective without trying to fit the model into the logistic equation format.

The second contribution is that a rigorous approach from an empirical chaotic perspective is applied to commodity prices, which to this point has not been forthcoming. This includes the use of neural networks. In several instances, problematic issues surrounding testing and questions of robustness, which have not been entertained in previous studies, are addressed.

The final contribution is to forecasters who depend on correlations in the data to present meaningful predictions. This study identifies which prices have nonlinear structures and chaotic dynamics, thereby leading to improved forecasting ability. 


\section{CHAPTER 2: REVIEW OF LITERATURE}

Price forecasts are largely made by conventional econometric methods, with time series approaches occupying minor roles. Because of the dominance of agricultural economics, there has been an overemphasis on explanation, and little interest in the predictive power of model.

P.G. Allen (1994)

\section{Introduction}

In the previous chapter, it was documented that internationally traded agricultural prices are volatile. This volatily has led to difficulty in the accurate forecasting of the prices (Allen, 1994). Because of this inherent inaccuracy, researchers and forecasters alike have sought to determine the appropriateness of various economic and statistical models to these data. Consequently, both the theoretical and empirical development of the commodity price behavior has ranged from the early work on deciphering temporal price behavior to recent studies that embody tests of market efficiency and uncertainty. From the outset, the commodity price literature has attempted to develop and estimate empirical models that were representative of current economic theories, mostly the efficient market hypothesis.

The efficient market hypothesis $(\mathrm{EMH})$ and the associated capital asset pricing model (CAPM), as discussed later in this chapter, represent the established view of how, in general, all economic prices behave. Because of this, proponents of the EMH determined that any type of market forecasting was a waste of time (see Allen, 1994).

In general terms, competitive agricultural commodity prices and processes generate quantities. The large numbers of farmers and buyers deal with a quasi-homogenous product and are regarded to be price takers. Because of the biological nature of the production process, lags 
can be represented in terms of months and even years which tend to result in small short-run supply response elasticities.

Government polices play an important role in agricultural markets. Tyers and Anderson (1992) have concluded that policy interventions in these markets significantly affect both long run average price and the volatility of prices in general. As a consequence of the important role commodity prices play in the incomes of developing countries, world markets for agricultural products result in more volatile international market prices as a consequence of domestic and agricultural and trade policies under free trade agreements.

Therefore, the intent of this review is to initiate the reader into the substantive development of commodity price behavior and models from both empirical and theoretical perspective. This detailed exposition into agricultural price behavior would not be complete without first examining agricultural price determinants. That is, examining the various pricing mechanisms that generate agricultural price series.

\section{Price Mechanisms}

To provide an intuitive understanding of the economic factors that generate increases in commodity price fluctuations, a brief identification of price mechanisms is required. These mechanisms can be categorized into five institutional arrangements:

- The negotiation of an informal or collective agreement between firms and individuals;

- The organized trading on exchanges or auctions;

- The formula pricing;

- The collective bargaining between producer groups, including cooperatives, and first handlers or buyers and between governments or government agencies; and

- The administrative decisions both by private firms and by public agencies. 
These pricing mechanisms most often are commodity specific. For example, US pricing arrangements for fresh fruits and vegetables, hay and nursery products fall mainly within the first category. Alternatively, price mechanisms for grains, soybeans, cotton, tobacco, and types of livestock and livestock product and the prices selected for this study are the result of competition or auctions on commodity exchanges, especially those for future contracts. Eggs, broilers, wholesale beef, and milk sold under federal and state marketing orders are examples of formula pricing. ${ }^{13}$

Next, the price mechanism of collective bargaining holds a relatively minor status as the pricing mechanism for agricultural commodities in the US. At the producer level, some fruits and vegetables sold to processors are priced in this way. ${ }^{14}$ However, on the international scene, producer organizations and/or governments, for the reasons alluded to in Chapter 1, have desired at various times to establish floor and ceiling prices for internationally traded commodities such as sugar and coffee.

Finally, administered prices are utilized in a variety of circumstances. The outstanding trait of this price mechanism is that a regulatory agency or firm can or has the ability to establish the price; however, market forces generally play a determining role in the formation of the price. ${ }^{15}$

\footnotetext{
${ }^{13}$ Formula pricing takes many different forms. Prices paid to sellers may be linked (via a formula) to a quoted price, say from a trade publication or to a price prevailing in a previous period. In the latter case, the historical or base price is then adjusted to reflect changes in costs or other factors affecting supply and/or demand. Thus, formulas have two components: establishing the base price and determining the linkage of the base price to the transaction price.

${ }^{14}$ The price premiums for milk sold for fluid use may be negotiated by bargaining between farmer cooperatives and milk handlers.

${ }^{15}$ For example, a firm cannot maintain a price greatly different from that established by competitors, even though it does have the discretion to establish prices somewhat above or below those of competing products or services.
} 
The ability to fix prices unilaterally does not exist. Farmers are price takers. As mentioned previously, administered prices for agricultural products in the US take the form of minimum support prices established under government programs. Obviously, these prices can and in some instances do influence market prices, but not necessarily determine them. The role government then plays in the determination of market prices in the US for commodities is important. $^{16}$

Two types of trading occur on organized markets. One is the "spot" or cash market, which involves trading in actual commodities, normally based on samples. The other is in futures contracts, which determine the minimum grade or particular grades of a commodity that must be delivered in fulfillment of a future dated contract. In markets where both types of trading take place, the near futures price serves as the basis for negotiating the cash prices paid for specific lots of commodities; buyers and sellers negotiate cash prices at discounts or premiums from the near futures with differences based on the grade, moisture content, etc. of a particular lot of grain. Even when cash transactions do not take place in a central market, the futures market is often used as a reference for pricing.

The major advantage of organized exchanges is that they provide an impersonal method of pricing that typically is not subject to the whims of either buyers or sellers. That is to say they perform an important discovery function to determine the level of prices that will equate shortrun demand and supply. However, a major criticism leveled against commodity markets is that prices tend to fluctuate "excessively" due to expectations, perhaps irrationally, in response to rumors or mass psychology. Unfavorable weather, for example, can induce buyers to expect a 
rise in the price level, which then would attract other buyers who want to capitalize on a rising market? Alternatively, the reverse may occur in a failing market. Such changes in speculative demand can serve as a useful purpose to the extent that the expectations are correct. Problems arise, however, when expectations are wrong or exaggerated. They unnecessarily reinforce or magnify price changes, thereby leading to greater amplitude of fluctuations than required simply to clear the market or to allocate inventories. With the passage of time, markets tend to correct excessive changes in prices. Moreover, it is difficult to demonstrate empirically that prices in terminal markets have unwarranted runs.

Pricing mechanisms, especially auction-type markets, have also been criticized for contributing to price instability. One such market is the futures market, which serves a number of functions. The futures market is a major price discovery mechanism and provides an opportunity for producers and users of commodities to shift the risk of adverse price movements and forward price commodities. Commodity futures markets also offer an opportunity to speculate and perhaps, thereby, to gain from changes in prices.

Spot or cash prices reflect the value of commodities offered for immediate delivery. They are based on actual samples of commodities locally available. Spot prices reflect the unique characteristics of individual trade such quality and location of the lot being sold. Spot prices are an example of the data chosen for this work.

In contrast, futures prices are based on contracts to deliver a fixed quantity (and specific quality) of a commodity at approved locations at some future date. Thus, in buying or selling futures, one is dealing in contracts to deliver a commodity at a future date rather than the

${ }^{16}$ These prices have accounted for about one half of the total cash receipts of farmers. These included all the major cereal crops, soybeans, peanuts, cotton, tobacco, milk, sugar, honey and 
commodity itself, and since the contracts are standardized, the transactions need establish only the price and number of contracts traded.

Relationships between futures and cash prices for such commodities as wheat and corn are explored. These commodities are produced annually, but are stored and consumed throughout the year. They can be characterized as seasonally produced, continuous inventory commodities. Two aspects of the behavior of the constellation of prices are of interest: changes in overall level of prices and changes in cash-futures price differences.

The difference between a futures price and a cash price is commonly referred to as the basis. Both cash and future prices are influenced by changes in supply and demand. Assuming annual supply and demand remain stable, prices will still change from month to month, thus, abstracting from all but seasonal influences on prices.

In reviewing the factors that are considered to significantly contribute to commodity price instability, one would suggest the use of structural models. However, the dynamic nature and need for accurate short-run forecasts has led to considerable attention directed at the interplay between dynamics and stochastic in economic modeling. Therefore, it is important to examine some of theoretical modeling developments that have occurred.

\section{Theoretical Modeling of Agricultural Prices}

The last thirty years have seen a steady development of tools for dynamic analysis in economics, both in theoretical development and in empirical implementation. The theoretical equations were frequently nonlinear in form, but the empirical methods have largely employed the tools of linear stochastic analysis. The assumptions of randomness were made partly because 
no simple system fit the data very well and partly because of the subjective perception that the future was uncertain. The theoretical analysis of dynamic systems was largely devoted to the determination of their steady states or solutions, which exhibited steady (exponential) growth. In stochastic models, the equivalent of a steady state solution is a stationary or invariant probability distribution.

The economic theory underlying the dynamic systems tends to emphasize amplitudereducing behavior (negative feedback). The equilibrium dynamics of these models is characterized by difference equations. The original tendency of the research was to search for solutions, which were constant in time. But more recent research, partly influenced by the new methods of nonlinear dynamics analysis developed in physics and ecology, has demonstrated that there are solutions to the same equations with cycles and even with chaotic behavior.

Commodities are distinguished not merely by their usual characteristics and date, but also by the unfolding of the events that are uncertain today but will be resolved by the date at which the contract is to be realized. Corresponding to the difference equations which define the dynamic equilibrium in the case of certainty is a set of stochastic difference equations that replaced the search for steady states for stationary distributions. The analogues to cycles and chaotic behavior have not yet been studied.

As already observed, the standard equilibrium dynamics, though they appear to have many negative feedback elements, are nevertheless capable of exhibiting behaviors other than convergence to a steady state. In fact, it is not hard to find positive comments on elements in the real world. 
It is generally recognized that an understanding of expectation formation is crucial to modeling price formation in agricultural commodity markets. One reason for this importance is that primary commodity production typically exhibits long lags. This is most obviously true in mining where a new mine will have a lead-time of 7-10 years and in tree crops where trees only become productive 3-5 years after planting. Investment decisions in these industries therefore depend upon the expectations of market conditions likely to prevail many years ahead and the same factors may operate to a more limited extent on the demand side.

However, this observation is also true of a manufacturing industry. What distinguishes primary industries from manufacturing in this respect is their competitive structure (see e.g. Labys, 1980) that as the consequence of short-run elasticity of both demand and supply, results in substantial price volatility. Consequently, a primary commodity producing enterprise will need to form expectations of the prices it is likely to obtain, whereas, by contrast, a manufacturing enterprise will focus on likely levels of future demand.

The second reason for the importance of expectations formation in understanding primary commodity markets relates to the role of stocks (see Gnosh et al., 1987). Price variability, in conjunction with the high degree of product homogeneity, provides incentives to third parties to carry stocks forward in periods of low prices in the expectation of higher prices in the future. This activity raises prices in periods of excess supply and tends to lower prices in periods of excess demand, thereby providing a degree of automatic price stabilization (Samuelson, 1965; Wright and Williams, 1982, 1984). Active trading of many of the most important primary commodities on futures markets facilitates it. By taking an offsetting position the stockholder is 
able to substantially reduce the risks of the combined (stock plus futures) transaction and this will imply an increased willingness to carry stock (Gilbert, 1985).

For both of these reasons, current supply and demand (including demand for stocks) of a primary commodity will depend both on expected prices and on prices previously expected to prevail in the current period. A higher expected future price will raise current stock demand and thereby raise the current price. A higher expectation in the past of today's price will have tended to raise current supply and will suppress the current price.

The theory of commodity supply views supply as a function of producers' profits expectations (where producers maximize profits based on the relation of a commodity's price to its cost of production). In the short-run production, capacity is fixed and this places a limit on potential supply. However, in the long-run production capacity can be varied. Given the producers optimizing choice of the rate of capacity utilization, short-run supply may also be influenced by supply shocks such as unusually good or bad weather (especially in the case of agricultural products), labor strikes, and technological innovations.

Muth's Rational Expectations Hypothesis (REH) developed in Muth (1961) revolutionized economic theory and modeling on price formation in a simple agricultural market. REH states that in the formation of expectations about future economic conditions, economic agents use all the information available to them efficiently. The implication is that their expectations may be modeled as the mathematical expectations of the relevant variables conditional upon the information set available to those agents (see e.g. Begg, 1982 ). In the medium to long term, primary commodity prices are determined by the intersection of the 
commodity's consumption (demand) and production (supply) curves. In the short term, stockholding tends to even out price movements.

In a commodity price model, it is useful to distinguish between application of the REH to the "physical" production and consumption relationships and its application to how intertemporal stockholding affects short-term price determination. In practice, most econometric work has concentrated on the implications of the REH for stock and price relationships. ${ }^{17}$

\section{Empirical Modeling of Agricultural Prices}

The unpredictability of agricultural commodity prices has been explored empirically by the hypothesis of market efficiency. ${ }^{18}$ The efficient market hypothesis is a joint supposition that representatives hold rational expectations because they avert making forecast errors on the support of their prevailing information set. Working (1958) asserted that randomness was inherent in commodity markets because continuous flows of unlike information into these markets generated recurrent price irregularity, which provided no constant specification.

Because of the guise that weather renders in the yield of agricultural commodities, the random walk hypothesis seems very unlikely. ${ }^{19}$ The random walk hypothesis is formulated in the following model under the null hypothesis,

$$
\mathrm{p}(\mathrm{t})=\mu+\alpha \mathrm{p}(\mathrm{t}-1)+\mathrm{e}(\mathrm{t})
$$

17 In a survey of methodologies for the modeling commodity price behavior, Gilbert (1990) has been somewhat pessimistic in its assessment of the usefulness of rational expectations methods in modeling primary commodity markets, but at the same time some hopeful directions have emerged.

18 The seminal work of Bachieler (1900) developed the empirical implementation of the market efficiency theory. Working (1934) translated the concept into the thought of random walk.

19 The random walk model dictates that the agricultural prices over some predetermined sampling interval are uncorrelated and have constant variance. 
where $\mathrm{p}(\mathrm{t})$ is the current agricultural price at time $\mathrm{t}$ and $\mathrm{e}(\mathrm{t})$ is a white noise process with $\mathrm{E}[\mathrm{e}(\mathrm{t})]=0$ and $\mathrm{E}[\mathrm{e}(\mathrm{t}) \mathrm{e}(\mathrm{s})]=0$ where $\mathrm{s} \neq \mathrm{t}$. Inflationary pressures cause prices to drift upwards. This becomes the positive random walk model where $\mu$ is positive. Recall that all random walk processes require that irregularities in prices be permanent. It would not be envisioned that a less developed country's government would be sapient to consider commodity booms as permanent. For example, Houthakker (1961), and Larson (1960) were involved in rejecting the null hypothesis of randomness for corn, wheat and soybeans. Labys and Granger (1970), and Cargill and Rausser (1972) established evidence for a modified random walk process. Stevenson and Bear (1970) and Leuthold (1972) employed filter rules to confirm positive and negative price dependence to cast doubt on the validity of the random walk model. ${ }^{20}$

Samuelson (1965) expanded this perception by submitting that commodity future prices emulated a martingale process. The martingale process can be illustrated in the following scenario,

A player tosses a fair coin and wins some money if heads come up and loses the same amount of money if tails come up. Let $x(m)$ denote the amount of money held by the player at the end of $m$ tosses. The martingale property insists that the amount of money held on the $(m+1)$ th toss is on the average the player's current fortune, which is not affected by the previous history, thus capturing the notion that the game is fair. If the game is favorable to the player then we have a "submartingale" process.

The martingale property conveys a statistical process that is conditionally unpredictable, denoted by

$$
E[p(t+1) \mid I(t)]=p(t)
$$


where $\mathrm{p}(\mathrm{t})$ is the current price and $\mathrm{I}(\mathrm{t})$ is the information set reflected in current agricultural market prices. A sub-martingale process is illustrated by replacing the identity in (2.2) with

$$
\mathrm{E}[\mathrm{p}(\mathrm{t}+1) \mid \mathrm{I}(\mathrm{t})]>\mathrm{p}(\mathrm{t})
$$

The random walk hypothesis is more restrictive and requires that successive price changes are independent and identically distributed. An excellent survey of the links between the martingale hypothesis, the more restrictive random walk hypothesis and tests of market efficiency can be found in LeRoy (1989).

Incorporating these relative concepts into a measure of unpredictability, Sims (1984) defined that a price process $\mathrm{p}(\mathrm{t})$ is instantaneously unpredictable if and only if, as $\mathrm{s} \rightarrow 0$,

$$
\mathrm{E}[(\mathrm{p}(\mathrm{t}+\mathrm{s})-\mathrm{E}[\mathrm{p}(\mathrm{t}+\mathrm{s})])]^{2} / \mathrm{E}\left[(\mathrm{p}(\mathrm{t}+\mathrm{s})-\mathrm{p}(\mathrm{t}))^{2}\right] \rightarrow 1
$$

Thus, for an instantaneously unpredictable process, the prediction error is in the central segment of innovations over small intervals. Of course, if $\mathrm{p}(\mathrm{t})$ is a martingale, which is defined in (2.2) and $p(t)$ has finite moments, the above ratio is exactly one, i.e. $E[p(t+s)]=p(t)$. It is meaningful to perceive that (2.4) does not exclude predictability of higher order moments such as the conditional variance. For example, fix the step size $s$ and let $\mathrm{p}(\mathrm{t}+\mathrm{s})=\mathrm{p}(\mathrm{t})+\mathrm{e}(\mathrm{t})$ where $\{\mathrm{e}(\mathrm{t})\}$ is an ARCH process. ${ }^{21}$ It is easily shown that (2.4) is one for each s. This shows that although (2.4) excludes predictability of the conditional mean, it does not exclude predictability of the conditional variance.

20 These studies concentrated on futures market prices due to the nature of the market efficiency hypothesis. Non-random walk behavior for spot market prices has also been demonstrated in the empirical literature.

$21 \mathrm{ARCH}$ stands for autoregressive conditional heteroscedastic, i.e. $\mathrm{e}(\mathrm{t}+\mathrm{s})$ is distributed normal with mean zero and variance $h(t+s)=\alpha+\beta h(t)$, with $\alpha>0, \beta>0$ and $h(0)$ given and positive. 
Even though conditional means may be hard to predict, it may be possible to predict other conditional moments such as conditional variances, conditional skewness, and conceivably the conditional moment generating function. Current studies have focused on the predictability of the conditional variance, $\mathrm{V}[\mathrm{p}(\mathrm{t}+1) \mid \mathrm{p}(\mathrm{t})]$ which has resulted in testing for the presence of ARCH (autoregressive conditional heteroskedasticity) and GARCH (generalized autoregressive conditional heteroskedasticity) effects in commodity price models. ${ }^{22}$ For example, Yang and Brorsen (1992) have substantiated the pertinence of the GARCH model for corn, pork bellies, soybeans, sugar, wheat and gold daily price changes. Abandoning the linear corn/hog or price cycle analysis of Jameson (1983), Chavas and Holt (1991) affirmed the validity of the GARCH model and submitted that the dynamics of the pork market may be nonlinear.

Wolff (1988) has disclosed that there exists a resemblance between the conditional variance of a random coefficient model (RCA) and the ARCH model of Engle (1982). He confirms that for every ARCH model there is a random coefficient model which implies exactly the same configuration for the conditional variance of the variable of concern. Furthermore, Engle (1982) states,

The existence of an ARCH effect would be interpreted as evidence of misspecification either by omitted variable or through structural change.

Directing on the issue of structural change, the $\mathrm{ARCH}$ or RCA is quite amenable to the above scenario. It can be argued that the findings presented above illustrate that these effects mentioned by Engle have occurred in agricultural commodity prices. For example, the period of

${ }^{22}$ A distinction should be made here between the ARCH or GARCH effect and the ARCH or GARCH model. The ARCH/GARCH effect is the presence of heteroscedasticity in the residuals 
1973-74 represented different market forces than the sixties or eighties. If the response of agricultural prices in subsequent periods of substantial excess supply had matched the response to the relatively modest excess demands in that period, agricultural prices in the 1980s would have been even lower than observed. It would be expected that the parameters of models which explain the eighties cannot explain the mid-seventies. ${ }^{23}$

Deaton and Laroque (1992) and Gilbert (1985) have developed models specifying nonlinear conditional means for agricultural commodity prices. This economic argument for the use of nonlinear conditional means was given by Gilbert (1990).

So long as positive stocks are held, the commodity price varies in a random walk manner about a trend rising at the rate of interest (i.e. follows a martingale process with positive drift). Over time this drift will take the price above its long-term mean to an increasing extent. There will, however, always be the possibility of a sufficiently large negative shock (i.e. a fall in production or rise in consumption) as to result in stock-out. That shock will result in a further and possibly sharp rise in the price but will also break the connection between the current price and the price in succeeding periods. Hence, after stock-out there will be a new price path, also drifting upwards at the rate of interest, but starting at a lower level.

The success that these models have had in modeling empirical agricultural commodity prices demonstrates the consequence that a supply shock will have a significantly smaller effect on the commodity price when the availability is low. Gilbert (1990) states,

The standard linear model fails to account for primary commodity price movements in any significant area, so it is important to do empirical work to learn to which commodities this nonlinear model applies.

of linear time series models while the ARCH/GARCH model is the specification that removes the effect.

23 Since most commodity market modeling has established trust in the linear paradigm, one would expect that structural change has transpired. This dispenses testimony of structural change. 
If agricultural markets are nonlinear, the possibility exists that price trajectories generated by market interactions are absolutely predictable granted that measurement of market interactions is without error. However, imperceptible errors can be heightened to macroscopic proportions so that these trajectories would exhibit random behavior over longer time scales. ${ }^{24}$ In other words, randomness emerges from determinism and forecasts of future prices can become trivial at an exponential rate. This attribute of nonlinear dynamics, chaos, occurs when, on the average, nearby trajectories separate at an exponential rate. This is dissimilar to a cause attributed to evident randomness known as complexity. Complexity is defined as behavior that involves many irreducible degrees of freedom. Alternatively, complexity is measured in terms of the dimension of the system's trajectories that should be the same as the number of irreducible degrees of freedom.

Chaos and complexity do not preclude each other; chaos also occurs in systems with many degrees of freedom. These trajectories of many complex systems, asymptotically, fold onto only a few degrees of freedom; thus, losing much of its complexity, while maintaining chaos and randomness. Behrman (1986) implicitly remarks on this folding process in the following comment,

International commodity markets are complex organisms, often with a large number of actors directly or indirectly involved in production and consumption. Models cannot usefully duplicate this complexity and detail. Attempts to do so are doomed to failure because of the enormity of the task. Instead good modeling requires simplifications and abstractions to represent the essential elements of the system under examination. p.9

It is possible to find low-dimensional chaotic behavior mixed together with high dimensional complex behavior. Recent studies by Brock and Scheinkman (1989) and Brock and 
Sayers $(1986,1989)$ suggest that some economic times series are low-dimensional chaotic processes. ${ }^{25}$

Deaton and Laroque (1992) explain that while progress has been made toward a theory of commodity price determination, little or no research has addressed the question of whether that theory can explain the actual behavior of agricultural prices.

${ }^{24}$ Farmer and Sidorowich depict that anyone can predict the motion of a roulette ball by eyeing a second into the future, but predicting it fifteen seconds into the future is difficult even with advanced quantitative methods.

25 Other empirical studies are Brock (1987), Barnett and Chen (1989a and b), Frank and Stengos (1989), Frank and Stengos (1988a,b). Theoretical discussions on the applicability of chaos to economics can be found in Baumol and Quandt (1985), Benhabib and Day (1980), Chiarella (1988), Savit (1988) and Stutzer (1980). 


\section{CHAPTER 3: A BRIEF REVIEW OF CHAOS THEORY}

Why have the meteorologists such difficulty in predicting the weather with any certainty?... Here again we find the same contrast between a very slight cause, inappreciable to the observer, and important effects, which are sometimes tremendous disasters.

H. Poincare (1946)

\section{Introduction}

Integrating dynamical behavior in economic theory is not of recent origin. However, the incorporation of chaotic dynamics into econometric applications is. The first exploration of “chaos" in economics was developed by Benhabib and Nishmura (1979) when they used May's (1976) celebrated logistic function. They investigated theoretical models characterized by the dynamical behavior of first-order nonlinear difference equations. Other related conceptual developments and empirical work was followed by Baumol and Quandt (1985), Benhabib and Day (1980, 1981, and 1982), Chiralla (1988), and Stutzer (1980).

Brock (1986) built on the work of Grassberger and Procaccia (1983a and b), Wolf et al. (1985) and Eckmann and Ruelle (1985), and developed associated statistical measures to determine if empirical economic time series displayed chaotic properties. Brock and Baek (1991) develop asymptotic tests for these measures.

For those unfamiliar with the implications and theoretical developments of chaos theory, this chapter examines many of the important definitional assumptions as well as the modus operandi of the theory. The attempt here is to provide a cursory examination of the field of chaos theory which necessary to proceed with the theoretical and empirical results of subsequent chapters. $^{26}$

\footnotetext{
${ }^{26}$ For a complete mathematical discussion, refer to the work of Devaney (1984). Financial implications of chaos theory can be found in Varga (1994).
} 
As stated in previous chapters, over the past few decades a fundamental shift has occurred with respect to modeling economic systems. ${ }^{27}$ The reliability of past assumptions has begun to be closely scrutinized. No longer restricted to the confines of traditional linear dynamical systems, economists have shifted their attention to the interplay between non-linearity and predictability. While predictability is of paramount concern to those in any scientific endeavor, careful scrutinizing of the properties of predictability with stochastic systems has only recently come into vogue.

Recent developments in nonlinear dynamics have produced deterministic explanations for phenomena that have previously been viewed as inherently stochastic. Some chaotic dynamical systems, although deterministic, produce solution paths that appear to be stochastic. In fact, a stochastic explanation is indeed natural in such cases and is produced by defining a probability measure over the limiting range set, despite the fact that the system that produced the path is entirely deterministic. There is a more formal definition that a time sequence is chaotic if the sequence has the following three properties:

- sensitive dependence on the initial conditions,

- a form of stationarity, and

- non-periodicity

The essence of chaotic behavior belongs to what is known as a set called an Attractor. The geometrical form of these attractors, and especially those attractor sets which posses a non-integer valued or fractal dimension known as strange attractors, plays an important role in chaotic

\footnotetext{
27 The impetus for current applications in chaos theory is an outcome of the work done by Poincare in celestial mechanics in the 1940s along with research done by G.D. Birkhoff in the new field of ergodicity. The 1960s and 1970s witnessed the beginning of the theory through the empirical work of Edward Lorenz in meteorology and theoretical work contributed by Stephen Smale, Robert May, Tien-Yien Li and James A. Yorke.
} 
dynamics. For example, collect together the cluster (limit) points of the sequence $\{x(t)\}$ into a set $\mathrm{X}$ called the attractor. In the limit, $\{\mathrm{x}(\mathrm{t})\}$ will be trapped, or locked in, by that attractor set if the initial value $\mathrm{x}(0)$ is sufficiently close to the attractor set. Once trapped by the attractor (i.e., for sufficiently large t), the trajectory will wander within or near the attractor set forever, and the behavior of the trapped paths within the attractor set will be influenced by the geometry of the attractor set. Strange attractors are typically fractal, having non-integer dimensions and zero volumes.

If we believe that the dynamic evolution of economic variables can be modeled by a finite number of factors, then we can use the recent developments in nonlinear dynamics to explain it. It follows that it should be possible empirically to recover some information about unknown dynamical systems from the observed path of economic variables. We should expect that the resulting unknown dynamical system will produce observed paths that appear to be stoachastic, and conditional subsystems that appear to be subject to stochastic shocks from outside the subsystem. Potential exceptions to these conclusions could result from the existence of non-negligible pure white noise induced by measurement error. As a result, data quality is of great importance in empirical applications of mathematical chaos to economics. One of the advantages of using chaotic dynamics is that no assumptions are needed about the depth of modelling needed to endogenize the system's dynamics and, hence, does not depend on one's view about the source of the system's dynamics.

Before the existence of chaotic behavior in economics can be ascertained, several assumptions have to be made concerning the dynamics of the data generating mechanism. The first is that the system is non-linear, that is, the system has non-linear constructs. The second is that the 
system is dissipative. A dissipative system in an economic context can be described as a self-regulatory mechanism, that is, a system that has loss mechanisms that dampen the response to incoming stimuli. Effectively, the dynamics of a system of a non-linear dissipative system can be examined by systems of non-linear, difference relationships.

Because nonlinear dynamical systems are capable of behavior that is erratic and unpredictable, these systems have been labeled as chaotic dynamics or simply chaos.

\section{Chaotic Systems}

To proceed further, a descriptive mathematical description of the property of chaos is warranted. Because of the recent proliferation of articles on chaotic behavior, the attempt made here is to use the restrictive assumptions of Devaney (1989) to start our journey. Initially, our understanding of chaotic behavior is contingent upon a mathematical representation of a univariate mapping $f$ on the unit interval $[0,1]$ with the properties of topological transitivity, sensitive dependence, and dense periodic points. ${ }^{28}$

Devaney $(1989$, p.50) provided another definition of a chaotic function. He built the basis for his definition on the closed unit interval I. A function $f: I \rightarrow I$ is chaotic on $\mathrm{I}$ if and only if $\mathrm{F}$ is

- topologically transitive

- sensitive dependence on initial conditions, and

- periodic points of $\mathrm{F}$ are dense in I

Topological transitivity means that any subinterval of I contains at least one point whose orbit enters any other subinterval of I. Specifically, $f: I \rightarrow I$ is topological transitive if for any two

\footnotetext{
${ }^{28}$ It is the property of sensitive dependence that is familiar to many economists.
} 
open sets $\mathrm{U}$ and $\mathrm{V}$ in I there is a $\mathrm{k}$ such that $f^{k}(U) \subseteq V$ where $\mathrm{k}$ refers to the kth iteration of the mapping $f(\cdot)$.

Quite often referred to as the landmark of chaos, sensitive dependence on initial conditions requires that there be a distance $d$ such that for any point $x_{0}$ an interval containing that point contains some other point $\mathrm{x}_{0}{ }^{\prime}$, whose orbit eventually separates from the orbit of $\mathrm{x}_{0}$ by a distance greater than $\mathrm{d}$.

Heuristically, sensitive dependence means that there is a constant $\delta>0$ such that, arbitrarily close to any point of $\mathrm{X}$, one can find another point of $\mathrm{X}$ whose orbit eventually diverges from that of the given point by more than $\delta$. As is discussed later, the Lyapunov exponents are used as a practical measure of this divergence. Although this discrepancy in orbits is required only to exceed $\delta$, not to be arbitrarily large in absolute terms. It should be noted that the ratio of this discrepancy to the distance between $\mathrm{x}_{0}$ and $\mathrm{x}_{0}$ ' will become arbitrarily large when $\mathrm{x}_{0}$ ' is chosen arbitrarily close to $\mathrm{x}_{0}$. It is in this sense that sensitive dependence implies unpredictable long run behavior for orbits originating arbitrarily near one another. Finally, if every subinterval of I contains a periodic point of $\mathrm{F}$, then its periodic points are dense in the interval $\mathrm{I}^{29}$

Therefore, the concern for determining if a system exhibits chaotic behavior is that the map only operates on a closed and bounded interval, with any chosen initial point in the interval upon iteration leading to a unique periodic orbit. The collection of these orbits must represent every value in the interval.

This, of course, is quite different from the generally accepted economic principle of equilibrium. The term equilibrium as used in economics is to describe the ultimate destination of

\footnotetext{
${ }^{29}$ While three conditions are provided, it is only necessary for two to be met.
} 
an orbiting price, also called an attractor. For example, most often, equilibrium analyses of economic systems are based upon the familiar cobweb model. Recall that the cobweb type of dynamical system was discussed in Chapter 2. This dynamical system utilizes the evolution of linear difference equations that yield convergent, divergent, or periodic solutions. Geometrically, this model can be visualized as an object such as a fixed point or a closed curve. However, by graduating to the non-linear paradigm, it is possible for certain types of dynamical systems to converge to other geometrical objects such as Cantor sets. In this case the attractor has what is known as a fractal structure and is termed as a strange attractor.

Since it is possible for an economy to posses two or more equilbria, dynamical systems may also have more than one attractor. For example, when a set of initial points converge to an attractor, the result is known as a basin of attraction. Each attractor does have a basin and therefore, it is expected that in the example each basin would compete for the points. ${ }^{30}$ Because of this competition, potentially any change in the initial conditions may shift a point to one basin or the other, thereby changing the ultimate destination of the system. This is known as sensitive dependence on initial conditions and is one of the main features of chaotic systems. The existence of a fractal structure enables points that behave erratically and orderly to coexist.

It may be useful at this point to provide some mathematical expositions of the ideas so far presented. As mentioned, one of the features of linear dynamical systems is that they can asymptotically converge to a fixed point. For example, the orbit of a point, $x$, and a function $f$ with a convergent orbit, $x, f(x) f^{2}(x), \ldots, f^{n}(x), \ldots$ converges to the limit $L$ as $n \rightarrow \infty$. Alternatively, a system may converge to a fixed point that is the original point. For example, $f^{n}(x)=x$. Basically, 
the systems begin at some point and after $\mathrm{n}$ orbits it returns to $\mathrm{x}$ and then repeats the process. This is an example of the form of a closed curve of an attractor and is called a cycle of length $\mathrm{n}$. In this case, $\mathrm{x}$ is referred to as a periodic point of $\mathrm{f}$ with period $\mathrm{n}$. The smallest positive $\mathrm{n}, \mathrm{n}_{0}$ for which $f^{n}(x)=x$ is called the prime period of $x$. It can be easily shown that any period of $x$ is a multiple of the prime period.

Determination of the existence of the cycle of length $\mathrm{n}_{0}$ can imply the existence of other cycles. For example, Li and Yorke demonstrated that a continuous function mapping an interval J into itself, and if some point in $\mathrm{J}$ is periodic for $\mathrm{f}$ with prime period 3 , then for every positive integer $\mathrm{n}$, there is a periodic point in $\mathrm{J}$ having prime period $\mathrm{n}$. As a result, the existence of a cycle of length 3 guarantees that there must be cycles of all lengths.

In Devaney (1989), the Sarkovskii theorem demonstrated that if $\mathrm{f}$ is a continous function mapping the set of $\mathfrak{R} \rightarrow \mathfrak{R}$, then if $\mathrm{f}$ has a periodic point of prime period $\mathrm{k}$ and $\mathrm{k}$ ' is any integer $\mathrm{k}$ appearing later in the integer ordering, it follows that $f$ also has a periodic point of prime period k'. As a result, if $\mathrm{f}$ has any cycle whose length is not a power of 2 , then $\mathrm{f}$ must have cycles of infinitely many different lengths. This ordering is

$$
3,5,7, \ldots, 2 * 3,2 * 5,2 * 7, \ldots, 2^{\mathrm{n}} * 3,2^{\mathrm{n}} * 5,2^{\mathrm{n}} * 7, \ldots, 2^{*} 3,2^{*} 2,2,1
$$

The economic implication of this result is that if an economic system has a cycle of length 5 years, then for the initial points that model must be capable of generating cycles of infinitely many other lengths.

30 The boundary between these basins can also be fractal while the attractors are simple geometrical objects. 
The Schwarzian derivative is an important tool for determining the existence of a chaotic behavior of a function $\mathrm{f}$. For example, if the Schwarzian derivative is negative for all $\mathrm{x}$ then all interates of the function are also negative. In other words, in the presence of this assumption of negativity, the critical points of the function play an important role in determining the dynamics of the system. The Schwarzian derivative of a function $\mathrm{f}$ of $\mathrm{x}$ is

$$
S f(x)=\frac{f^{\prime \prime \prime}(x)}{f^{\prime}(x)}-\frac{3}{2}\left(\frac{f^{\prime \prime}(x)}{f^{\prime}(x)}\right)^{2} \leq 0
$$

Stability of dynamical systems depends on the dynamical behavior of the system. For example, a system is stable if small changes to the system's structure leave the system's dynamical behavior qualitatively unchanged. Weiss (1984) gives an excellent example of this concept and is briefly mentioned here. It is expected that parameters of existing econometric models change over time. It is possible that these changes can be ignored if the system is stable. However, if they are not, then any incorrect estimation procedure or measurement error for obtaining these parameters leads to a system with qualitatively different behavior.

\section{Methods of Analysis}

At present, there are three possible ways to analyze a dynamical system: bifurication theory, symbolic dynamics, and the kneading theory. Each one presents both advantages and disadvantages to the economist.

Bifurcation theory studies the dynamics of the system as values of the parameters change. For example, let $x^{*}$ represent a stable fixed point. If the parameter $\mu$ is increased in value to a fixed point, say $x^{*}$, splits into two stable fixed points of period two and the former fixed point is now unstable. If $\mu$ is increased furthermore, each of these two fixed points split again into new 
stable fixed points at some particular value $\mu_{2}$ and leave the two formerly stable fixed points unstable. This process can be continued until the forcing parameter reaches some upper bound. To ascertain the entire sequence of fixed points, graphical analysis can be used to derive the entire phase portrait for the system.

The method of symbolic dynamics relies on an abstract model of the system that includes the essential features of the system under investigation. This is accomplished by changing the nature of the interval of which the system operates and relying on the property of topological conjugancy to analyze the system. Before proceeding further an examination of the property of topological conjugancy is warranted.

\section{Topological Conjugancy}

Suppose that $\mathrm{X}$ and $\mathrm{Y}$ are any sets each to which has been assigned a distance measure. Then, a homeomorphism between $\mathrm{X}$ and $\mathrm{Y}$ is an inevitable function mapping $\mathrm{X}$ onto $\mathrm{Y}$ such that both the function and its inverse are continuous. Suppose $\mathrm{f}$ is a continuous function mapping $\mathrm{X}$ into itself and $\mathrm{g}$ is a continuous function mapping $\mathrm{Y}$ into itself. Then, $\mathrm{f}$ and $\mathrm{g}$ are topologically conjugate if there exists a homeomorphism $\mathrm{h}$ between $\mathrm{X}$ and $\mathrm{Y}$ such that for each $\mathrm{x}$ in $\mathrm{X}$

$$
h(f(x))=g(h(x))
$$

As with earlier definitions, topologically conjugate functions map corresponding points to corresponding points, exhibiting the same dynamical properties and may be considered dynamically equivalent. The method of topological conjugancy enables us to find a system with similar properties, i.e. a known chaotic system, and attempts to find the homeomorphism between the two systems. 
Returning to our discussion on symbolic dynamics, the approach is to represent each vector in $\sum$ as a binary vector. A shift operator $\sigma$ that maps the first term in the sequence $\sum$ to a new sequence whose ith term is the (i+1)st term of the original. Relying on the property of topological conjugancy between $\sigma$ and $\mathrm{F}$, an additional mapping $\mathrm{S}$ is formulated that maps the itinerary of $\mathrm{x}$ which is an element of $\sum$. The function $S$ is also topologically conjugate. Therefore, $S$ retains the information in F and each iterate sequence of 0's and 1's. This allows the iterates to be counted because each orbit of period $n$ is produced by shifting $s \mathrm{n}$ times. The beauty of the method is that there are precisely $2^{\mathrm{n}}$ ways of arranging 0 's and 1 's to form a finite string $\mathrm{s}_{0}, \ldots, \mathrm{s}_{\mathrm{n}-1}$; hence, $\sigma$ must have exactly $2^{\mathrm{n}}$ periodic points of period $\mathrm{n}$. Using this approach, it is straightforward to determine that the period points are dense in $\sum$ as well as possessing the characteristic of sensitive dependence on initial conditions.

Kneading theory extends the symbolic dynamics methodology by keeping track of the orbit of the critical point and thus enables one to handle many additional complications. By examining the genealogy of periodic points through the method of symbolic dynamics, one is then able to develop a complete picture of the dynamics of the system.

Thus, if we combine the results of bifurication theory and the negative Schwarzian derivative with the kneading theory we are able to demonstrate a complete topological or qualitative description of the transition from simple to complicated dynamics. For direct application of these theories Devaney (1987) on p.154 states that

One may describe completely the "genealogy" of any periodic point in a transition familywhere it is "born", which sequences are its (period-doubling) "ancestors", and which sequences are its "descendants". 


\section{An Example of Chaotic Dynamics: The Logistic Model}

The logistic model is the seminal model of chaotic behavior. Considerable attention has been directed at the properties of the model. It also serves as an excellent vehicle to demonstrate the methods of analysis presented in the previous section. For the purposes discussed earlier, a cursory examination is needed to demonstrate some of the important caveats of the model. The method utilized rests on the approach of bifurication theory.

Consider the following non-linear difference equation, commonly referred to as the logistic equation:

$$
\mathrm{F} \mu(\mathrm{x})=\mathrm{x}_{\mathrm{t}+1}=\mu \mathrm{x}_{\mathrm{t}}\left(1-\mathrm{x}_{\mathrm{t}}\right)
$$

where the domain of $\mathrm{x}_{\mathrm{t}}$ is restricted to the open interval $[0,1]$. The dynamical behavior of this equation is trivial for parameter values of $\mu<1$, because all trajectories are attracted to $\mathrm{x}=0$. However, for non-trivial dynamical behavior it is required that parameter values lie in the open interval $(1,4)$. One of the more interesting properties of equation (3.3) is that if the initial value $\mathrm{x}_{0}$ were zero or if during the course of iteration $\mathrm{x}_{\mathrm{i}}$ were to become zero or one, then subsequent values of $x_{i}$ would be zero forever. If for example, $x_{0}$ and/or $x_{i}$ would become greater than one, then the next iteration would give a negative value of $\mathrm{x}$ and subsequent iterations diverge toward $-\infty$. Because of these properties, the domain of $\mathrm{x}_{\mathrm{t}}$ is restricted to the closed unit interval.

Table 3.1 illustrates the different parameter domains for $\mu$ in equation (3.3) and the resulting different forms of dynamical behavior exhibited by these domains. For example, in region I, for $0<$ $\mu<1, x_{t} \rightarrow 0$ for large $t$ while for region II, the systems converges to an equilibrium value for $x^{*}=(\mu$ - 1)/ $\mu$ (a simple attractor). In region III, the initial oscillations do not reach an unique equilibrium value or simple attractor like region II but approaches a permanent, periodic behavior. For 
example, if $\mu=3.3, x_{t}$ oscillates between 2 values or attractor values, 0.82360 and 0.47943 . If we change $\mu$ to $\mu=3.5$, then we move into a 4 period range, that is $x_{t}$ oscillates among four values, $0.87500,0.38282,0.82694$, and 0.50088 . As $\mu$ is increased further toward the value of 3.56994 , an infinite number of this period doubling occurs, that is, an infinite number, $2^{\mathrm{i}}$, where $\mathrm{i}$ is an integer enables the subdivision of region III. In the last region, region IV, the system is chaotic because of the infinite number of values that $x_{t}$ can possess. This demonstrates the non-existence of a periodic attractor.

The interval $[0,1]$ itself can be regarded as an attractor, that is, a "strange attractor". A strange attractor surfaces when there are increasingly many attractors which the system visits in turn before returning to its starting point. This $\mathrm{n}$-cycle, as we call it, will be complicated and as $\mathrm{n}$ increases to infinity, it is as if the motion was aperiodic. Since the equation that gives rise to the motion of the time series $x_{t}$ is deterministic, we do not have randomness in the pure sense of the term. Therefore, when equation (3.3) has a $\mu$ value in the chaotic region then the function has deterministic chaos. 
Table 3.1 Summary of Characteristics Associated with Equation (3.3) -the Logistic function , $\mathrm{x}_{\mathrm{t}+1}$ $=\mu \mathrm{x}_{\mathrm{t}}\left(1-\mathrm{x}_{\mathrm{t}}\right)$

I. $\mathrm{X}_{\mathrm{t}}->0$

II. $\quad x_{t} \rightarrow \mathrm{k}$ (i.e. non-zero constant)

III. $\mathrm{x}_{\mathrm{t}}$ oscillates between $2^{\mathrm{n}}$ values depending on the value of $\mu$ $0<\mu \leq 1$

$1<\mu \leq 3$

$3<\mu \leq 3.56994$

$3.56994<\mu<4$

Devaney (1989) has demonstrated that these attractors "bifurcate" - a stable attractor splits into 2 attractors, i.e. a 2-cycle - at a value $\mu=\mu_{\mathrm{n}}$ to an n-cycle, then

$$
\lim _{n \rightarrow \infty}\left(\mu_{n}-\mu_{n-1}\right) /\left(\mu_{n+1}-\mu_{n}\right)
$$

the ratio in (3.4) tends to the value 4.669201660910299 ....as $n \rightarrow \infty$ a number that is unique not only to equation (3.3), but also for other non-linear difference equations that imply this same period-doubling route to chaos.

One more note concerning the $\mu$ value in the chaotic region is the extreme sensitivity of the system to initial values, $x_{0}$. This sensitivity manifests in the form that successive iterates of $x_{t}$ depend on the value of $x_{0}$ chosen. A small change in the initial values can lead to entirely different time paths of $x_{t}$. One will notice for the other regions, I-III, that the time path of $x_{t}$ is completely independent on the initial value, $\mathrm{x}_{0}$. 
Since the attractors discussed above can be denoted by a point, i.e. an equilibrium value; a circle, 2-cycle; a torus, 3-cycle and so forth, topology which is the study of geometrical forms can be used to characterize an experimental time series.

\section{Conclusions}

It is now known that seemingly erratic long-run behavior and various forms of sensitivity to initial conditions can arise in even the simplest non-linear models. Unless there is substantial economic rationale for a priori specification of linear economic models, the process under investigation must be viewed as at least potentially exhibiting the type of chaotic behavior addressed in this chapter.

Chaos theory suggests that the long-range prediction of nonlinear economic processes may be subject to the same basic mathematical limitations as long-range weather prediction. In both cases, future behavior may appear independent of the initial conditions that precede it. 


\section{CHAPTER FOUR: AGRICULTURAL MARKET DYNAMICS}

What is twisted cannot be straightened, what is lacking cannot be counted. -Ecclesiastes 1:15(NIV)

\section{Introduction}

The previous chapters have shown that modeling agricultural price behavior is in a state of transition. This transition is from linear structural approaches to one that is non-linear. While the development of chaos theory and its associated influence on prediction is interesting, it is important to also view the theoretical context in which agricultural markets are to be analyzed. Therefore, it is important to look at the development of dynamical analysis in agricultural markets to ascertain the theory's utility.

In 1938, Mordecai Ezekeil stated,

... Classical economic theory rests upon the assumption that price and production, if disturbed from their equilibrium, tend to gravitate back toward normal. The cobweb theory demonstrates that, even under static conditions, this result will not necessarily follow. ${ }^{31}$

Early in the seventies, Nerlove (1974) examined the implications of these remarks and determined that in some circumstances, no matter how improbable it may be, price instability could result. In this illustrative example, it is demonstrated that in the absence of price support, wheat prices may be characterized by continuous instability. To further characterize this instability, Turnovsky (1985) introduced additive stochastic elements into the linear demand and supply equations of both Ezekiel (1938) and Nerlove (1974) and obtained stochastic stability conditions for their equilibrium models. An interesting result of this research is that

... to ensure stability, variances must be explicitly taken into account and this results in a tightening of the deterministic conditions. Furthermore, as $\sigma_{t}^{2} \rightarrow 0$, the stochastic and deterministic conditions coincide.

31 For example, consider Ezekiel (1938) and the cobweb theorem. 
Turnovsky (1985) also implies that a heteroscedastic variance is present in all of his linear equations. For our purposes here, this development is assumed for now to be deterministic and non-stochastic; however, stochastic elements are introduced in the final section.

\section{Models of Agricultural Prices}

Relaxing the assumption of linearity in models of agricultural prices has led to the identification of limit cycles, chaos, self-excitation, asymmetric distributions, leptokurtosis, sudden jumping behavior, time deformation and irreversability for these prices. An important implication of non-linear time series analysis is that when modeling for nonlinear relationships, it is necessary to consider not only the first and second moments of the data but also third moments. For example, the lack of normality and its associated linearity leads to a non-zero skewness coefficient. Therefore, the distributions of these empirical price series may take on characteristics dissimilar to normal stochastic processes.

Convention dictates the assumption that the dynamics of agricultural prices are an outcome of an international competitive market system. The empirical implementation of this assumption was restricted to the class of linear processes that restricted the topology of market dynamics. Several authors, through the use of the cobweb model and restrictive assumptions on

demand and supply have demonstrated that chaotic price behavior can occur. ${ }^{32}$ Arstein (1983) and Jensen and Urban (1984) demonstrated that if one of the supply or demand functions is nonmonotonic, specifically, quadratic, then chaotic price behavior can occur. Specifically, this non- 
monotonicity surfaced in the form of backward bending supply curves and multiple value demand curves. The basis for their conclusion was established through the logistic specification of the reduced form price equation of the cobweb model. For example, Cugno and Montrucchio (1984) utilized the tent map in a modified version of the cobweb model in their attempt to demonstrated chaotic behavior. Holmes and Mannin (1998) showed that chaotic price behavior is possible given a s-shaped nonlinear monotonic supply function coupled with a linear demand function under adaptive expectations. ${ }^{33}$ One feature that is common to both studies is that the first order derivative of the reduced form price equation is non-constant. Since chaotic price behavior requires nonlinearity in the model specification, non-constancy of derivatives is expected. To further understand these features and their relation to agricultural commodity price models, a context is needed in which to operate.

Labys (1973) has developed a general structural model for a competitive agricultural commodity market. The following equations describe the structural relationships for consumption, production, and price inventory conditions as well as a market clearing identity:

$$
\begin{aligned}
& \mathrm{c}(\mathrm{t})=\mathrm{f}\{\mathrm{p}(\mathrm{t}), \mathrm{p}(\mathrm{t}-1), \mathrm{y}(\mathrm{t}), \mathrm{Z}(\mathrm{t}) ; \mathrm{e}(\mathrm{t})\} \\
& \mathrm{q}(\mathrm{t})=\mathrm{g}\{\mathrm{p}(\mathrm{t}), \mathrm{p}(\mathrm{t}-1), \mathrm{Z}(\mathrm{t}) ; \mathrm{w}(\mathrm{t})\} \\
& \mathrm{p}(\mathrm{t})=\mathrm{h}\{\Delta \mathrm{s}(\mathrm{t}), \mathrm{Z}(\mathrm{t}) ; \mathrm{u}(\mathrm{t})\} \\
& \Delta \mathrm{s}(\mathrm{t})=\mathrm{s}(\mathrm{t})-\mathrm{s}(\mathrm{t}-1)=\mathrm{q}(\mathrm{t})-\mathrm{c}(\mathrm{t})
\end{aligned}
$$

${ }^{32}$ The cobweb model provides a theoretical explanation of the cyclical components of certain price-quantity paths through time. Ezekiel (1938) and Tomek and Robinson (1977) have contributed to the development and various extensions of the cobweb model.

33 Tomek and Robinson (1990) demonstrate that it is quite probable that some agricultural commodities have s-shaped supply functions. They argue that the shape of the supply curve can vary among commodities according to the slopes of individual firm marginal cost functions, profitable alternatives for resources, and capacity constraints. 
where $\mathrm{p}(\mathrm{t}-1)$ is the price of the commodity for one previous time period, $\mathrm{Z}(\mathrm{t})$ is a vector of exogenous variables, $\Delta \mathrm{s}(\mathrm{t})$ measures a one period change in the level of inventories, $\mathrm{c}(\mathrm{t})$ is the level of quantity demanded, $q(t)$ is the level of quantity supplied, $y(t)$ is the level of income and $\mathrm{e}(\mathrm{t}), \mathrm{w}(\mathrm{t})$, and $\mathrm{u}(\mathrm{t})$ are stochastic variables. Empirically, the sampling interval will make a major difference in the specification of the consumption and production lags (Labys, 1973). For example, if the sampling interval is relatively relative to the lags of production and consumption short, i.e., quarterly or monthly, an additional assumption is needed which requires that inventories vary considerably. Under this assumption inventories play a pivotal role in commodity price formation. ${ }^{34}$

\section{The Importance of Inventories}

Given interest in the short-run dynamics of commodity prices, the specification of inventory behavior in (4.4) becomes crucial. One has the choice of examining inventory behavior through the concepts of flow versus stock adjustment processes. The flow adjustment process is one wherein periods of excess supply or excess demand lead to a change in prices that invariably return to their equilibrium level. The stock adjustment process is concerned with the actual level of inventories instead of their change. This dictates that adjustments in commodity markets toward equilibrium are the result of stocks. ${ }^{35}$ Samuelson (1965) demonstrated that when stocks are held, the commodity price will increase by an amount equal to the cost of storage. French et al. (1985) has stated that producers hold inventories for a period in which the expected capital

\footnotetext{
${ }^{34}$ An important rule is that often the data must be replaced with surrogate data or variables due to the paucity of data. Especially, in the case of monthly and daily sampling intervals.
} 
gain is greater than the sum of the interest cost, the marginal storage cost (MSC) and the cost of bearing commodity price risk. This marginal storage cost is equated to the physical cost of storage that includes items such as rental changes and insurance premiums, minus the marginal convenience yield. French (1986) also assumes that the physical storage cost is a function of the current commodity price, $\mathrm{p}(\mathrm{t})$, and that the marginal convenience yield has an inverse relationship with the inventory level. Therefore, in between harvest periods a decrease in the marginal convenience yield generates an increase in the marginal storage cost. That is to say, when inventory levels are high, the marginal storage cost is also high thereby increasing commodity prices. Although this provides some insight into the relationship between commodity prices levels and inventories, it not meant to be exhaustive.

Furthermore, Labys, et al. (1991) has stated that

The dynamic lags between a specific market variation and the response to that variation are thus influenced by biological and physical constraints as well as economic factors associated with uncertainty and partial adjustment. The interaction of these elements frequently results in both price and quantity cycles for many agricultural commodities...The length of the biological process does not uniquely dictate these cycles but is a major determinant. Consequently, agricultural commodity cycles are not of fixed length.

Tomek and Robinson (1990) point out that at low inventory levels, prices in one period are expected to be insulated from shocks in another period, so there are large expected price changes. At higher inventory levels, the marginal storage cost is not very sensitive to changes in the inventory, and price shocks are transmitted freely from one period to another.

French (1986) speculates that these relationships generate large seasonal variation in the prices for agricultural commodities with relatively high marginal convenience yields at low

35 Demand for agricultural commodities tends to be price-inelastic in the short run (Labys and Lord, 1992). 
inventory levels and low convenience yields at high inventory levels. The extent of this seasonal variation is variable across commodity groups. ${ }^{36}$

Deaton and Laroque (1992) continue in this tradition and contend that an inventory holder expects a profit or capital gain to carry an inventory into the next period. Producers hold zero inventories if there is an expected loss, while if there is an expected profit, speculators will demand positive inventories. This has the effect of bidding up the price until current and expected future prices are equal. Therefore, it is possible to have two different relationships for commodity price behavior in (4.7), i.e., inventories are zero or positive. Therefore, when the previous period's price is low with respect to some threshold value, we can expect that these will be some form of inventory accumulation. Alternatively, in the presence of high prices, we would expect inventories on hand to decline before the next period's harvest.

As an illustration consider the following alternative specification of (4.1)-(4.4):

$$
\begin{aligned}
& \mathrm{c}(\mathrm{t})=\alpha_{0}+\alpha_{1} \mathrm{p}(\mathrm{t})+\alpha_{2} \mathrm{p}^{\mathrm{e}}(\mathrm{t})+\mathrm{e}_{1}(\mathrm{t}) \\
& \mathrm{q}(\mathrm{t})=\beta_{0}+\beta_{1} \mathrm{p}^{\mathrm{e}}(\mathrm{t})+\mathrm{e}_{2}(\mathrm{t}) \\
& \mathrm{p}(\mathrm{t})=\gamma_{0}+\gamma_{1} \mathrm{p}(\mathrm{t}-1)+\gamma_{2} \Delta \mathrm{s}(\mathrm{t})+\mathrm{e}_{3}(\mathrm{t}) \\
& \mathrm{s}(\mathrm{t})=\mathrm{s}(\mathrm{t}-1)+\mathrm{c}(\mathrm{t})-\mathrm{q}(\mathrm{t})
\end{aligned}
$$

where the variables are as defined in (4.1)-(4.4), $\mathrm{p}^{\mathrm{e}}(\mathrm{t})$ is the expected price which is $\mathrm{p}^{\mathrm{e}}(\mathrm{t})=\mathrm{p}(\mathrm{t}-1)$, each $\mathrm{e}_{\mathrm{i}}(\mathrm{t})$ is white noise and un-correlated between equations. Under the assumption that two possible regimes exist for $\mathrm{p}(\mathrm{t})$, equations (4.5) - (4.8) become

$$
\mathrm{p}(\mathrm{t})=\mathrm{k}_{0}+\mathrm{k}_{1} \mathrm{p}(\mathrm{t}-1)+\mathrm{v}_{1}(\mathrm{t}) \quad \mathrm{p}(\mathrm{t}-1)<\mathrm{p}^{*}
$$




$$
\mathrm{p}(\mathrm{t})=\mathrm{z}_{0}+\mathrm{z}_{1} \mathrm{p}(\mathrm{t}-1)+\mathrm{v}_{2}(\mathrm{t}) \quad \mathrm{p}(\mathrm{t}-1) \geq \mathrm{p}^{*}
$$

where $\mathrm{p}^{*}$ represents the critical price level and switches from zero to positive inventories with

$$
\begin{aligned}
& \mathrm{k}_{0}=\left(1+\gamma_{2} \alpha_{1}\right)^{-1}\left(\gamma_{0}+\gamma_{2} \beta_{0}-\gamma_{2} \alpha_{0}\right) \\
& \mathrm{k}_{1}=\left(1+\gamma_{2} \alpha_{1}\right)^{-1}\left(\gamma_{1}+\gamma_{2} \beta_{1}-\gamma_{2} \alpha_{2}\right) \\
& \mathrm{v}_{1}(\mathrm{t})=\left(1+\gamma_{2} \alpha_{1}\right)^{-1}\left(\gamma_{2} \mathrm{e}_{2}(\mathrm{t})-\gamma_{1} \mathrm{e}_{1}(\mathrm{t})+\mathrm{e}_{3}(\mathrm{t})\right) \\
& \mathrm{z}_{0}=\left(\beta_{0}-\alpha_{0}\right) / \mathrm{a}_{1}, \mathrm{z}_{1}=\left(\beta_{1}-\alpha_{2}\right) / \mathrm{a}_{1}, \mathrm{v}_{2}(\mathrm{t})=\left(\mathrm{e}_{2}(\mathrm{t})-\mathrm{e}_{1}(\mathrm{t})\right) / \alpha_{1}
\end{aligned}
$$

Equation (4.9) is the specification for $\mathrm{p}(\mathrm{t})$ under the condition that inventories are positive, while (4.10) is the case when inventories are zero. Equations (4.9) and (4.10) together, coupled with additive stochastic elements, represent what is known as a threshold autoregressive model.

\section{Price Dynamics of the Threshold Model}

Threshold autoregressive models (TAR) has extensively been explored by Tong (1979, 1980, and 1983). They represent a finite set of possible autoregressive models in which a process may obey at any point in time, thresholds for the passage from one member of the set to another. If the passage is determined by the location of the past data values relative to the thresholds, the TAR model is the piecewise linearization of a non-linear model over the state-space by the introduction of thresholds. Tong and Lim (1980) showed that the smooth exponential threshold autoregressive or SETAR model is general enough to capture certain features:

- limit cycles

- amplitude frequencies

- jump phenomena

The specification of these models can be written as

36 Commodities like corn that has one, relatively short, harvest a year is different from wheat 


$$
x(t)=\phi_{0}^{(i)}+\sum_{i=1}^{p} \phi_{i}^{(j)} x_{t-i}+e_{t}^{(j)} \quad \text { if } \mathrm{x}_{\mathrm{t}-\mathrm{d}} \in \mathfrak{R}(\mathrm{j})
$$

where $\left\{e_{t}^{(j)}\right\}$ is white noise with $e_{t}^{(j)}$ independent of $e_{t}^{(j)}$ for $j \neq j$ '.

Tong and Lim (1980) and Tong (1983) developed this model, which is non-linear in the variables and can exhibit chaotic behavior for certain values of the parameters. ${ }^{37}$ A simplifying assumption is necessary is to examine the above equations in terms of their conditional expectations. This removes the residual terms from equations (4.9) and (4.10). ${ }^{38}$ The model above can be further extended once we smooth the threshold requirement in (4.10). The exponential autoregressive model is an example of a two-sided smooth threshold autoregressive (STAR) model. For example, we have an exponential autoregressive (EAR) model of order p, written as

$$
x(t)=\sum_{i=1}^{p}\left\{\phi_{i}+\pi_{i} \exp \left(-\tau x_{t-1}^{2}\right)\right\} x_{t-i}=\mu+e_{t}
$$

When $\mathrm{p}=2$, Haggan and Ozaki (1981) has shown that both amplitude-dependent frequency and "limit cycle" behavior can occur in this discrete time series model.

Devaney (1989) demonstrates that the above model when it is restricted to the unit interval as specified by (4.9) and (4.10) is unimodal. Using kneading theory, one can demonstrate that in the presence of a negative Schwarzian derivative that places restrictions on

that has two longer harvests each year.

37 Devaney (1989) discusses the chaotic behavior of the threshold autoregressive model in terms of the tent map specification.

38 The additional assumption requires that the residual terms meet traditional econometric assumptions. 
the number and type of periodic points, we have the possibilty of chaotic behavior in (4.12). ${ }^{39}$ To facilitate this development, the assumption that $\mathrm{p}^{*}=1 / 2$ is imposed. That is, the midpoint of the interval serves to identify this transitional value. ${ }^{40}$ Let the following parameter specifications in (4.12) and (4.10) be $\mathrm{k}_{0}=0, \mathrm{k}_{1}=\mu, \mathrm{z}_{0}=\mu$ and $\mathrm{z}_{1}=\mu$. Devaney (1989) stated that for $\mu>\sqrt{ } 2$, the system is chaotic. However, for the interval $0<\mu<1$, the system has a unique fixed point.

Because we have some idea as to the sign of the parameters in the structural agricultural model of (4.5-4.8), it can be determined for the reduced form of the model. Clearly, $\alpha_{1}<0, \alpha_{2}<0$, $\alpha_{0}>0, \beta_{\mathrm{I}}>0$ and the values of $\gamma_{\mathrm{I}}$ are ambiguous. However, for the sake of exposition and clarity the assumption is made that $\gamma_{0}>0$. In addition to these signs, the tent map specification requires that $\mathrm{k}_{1}>0$ and $\mathrm{z}_{1}<0$. Because $\mu$ is represented in both equations, we can look at equation (4.10) to determine the applicability of the proposed model. Clearly, for $\mathrm{z}_{0}$ to be positive, requires that $a_{0}>b_{0}$. That is, autonomous supply must be greater than the level of autonomous demand. Because $\mu$ is represented in both cases, the difference between these autonomous levels must equal the difference in rates of change for the quantity demanded and quantity supplied. $^{41}$

In equation (4.9), $\gamma_{2}$ plays an important role in the dynamics of the system, that is, the marginal influence of the inventory change affects the demand and supply price elasticities of the

39 It is beyond the scope of this work to provide a detailed treatment of the theoretical constructs utilized here. Suffice it to say, the intent is to provide an illustrative example of the results of the theory to this empirical setting rather than address each specific issue.

40 The analysis is confined to the unit interval.

41 This seems to be a rather unrealistic assumption on the surface, but normalizing transformations to the unit interval coupled with a natural log transformation to interpret price elasticities make the units become less of a problem. 
system. Because of the number of parameters in the system, direct application of bifurcation theory is problematic.

Based on the specification of (4.9) and (4.10) we expect that at higher price levels the next period's price will be more volatile. For example, as the marginal level of inventory accumulation declines there are fewer inventories to influence price behavior. Therefore, agents become more susceptible to uncertainty. This results in a conditional variance that varies between the two regimes. It is an assumption that the conditional variance is constant between regimes. Therefore, it is expected that prices in the long run are to oscillate between these two regimens.

From a probabilistic standpoint, the tent map specification generates a bimodal distribution. That is, the system spends most of the time in either one of two separated states. The threshold between the two is an inaccessible region that is relatively unlikely to be occupied. 42

From the above analysis, for any $\mathrm{p}_{0}$ in the interval $[0,1]$ the correlation at lag $\mathrm{k}$ of $\mathrm{p}(\mathrm{t})$ is zero for any $\mathrm{k} \neq 0$. Thus, the spectrum is that of white noise. Furthermore, the introduction of a stochastic concept into a deterministic setting is one of the main features of chaos. In other words, these "chaotic" systems can imitate stationary AR(1) models as well other invertible functions.

Since deterministic processes can have white noise properties, i.e. comparable first and second moments, Liu et al. (1992) have labeled this white chaos. In the above example, Sakai

42 Certain transformation of stochastic variables can generate bimodal distributions. For example, the ratio of two standard normal random variables yields a bimodal probability distribution, (Cromwell, 1994). 
and Tokumaru (1980) have shown that for $\mu \neq 2$, the autocorrelations for the above system have an $\mathrm{AR}(1)$ representation. Because of the apparent duality between determinism and stochasticity, the task is to uncover the possible chaotic structure in the presence of noise. In fact, Liu et al. (1992) address this difficulty in their remark,

One can argue that statistical tests are not the proper way to detect deterministic processes. In this view, evidence of "strange attractors," say, is convincing enough. However, the sample sizes available from economic time series data are not large enough to provide such evidence. New techniques that could cope with small sample sizes are needed here as well. We are led to the conclusion that probabilistic methods are for the time being the most appropriate technique for analyzing economic time series data.

\section{Conclusions}

Building on the work of Deaton and Laroque (1992) it is possible to specify an agricultural commodity market model which is nonlinear and examine the dynamics followed by empirical validation of the model through testing for chaotic behavior in the empirical price series. Quite often the approach is to formulate a theoretical structural model and then estimate the parameters. Evidence for the acceptance of a theory is provided by the results of testing for the significance of the parameters from the structural model. This methodology pre-supposes that the testing framework is verified in the statistical literature. This is not the case for testing for chaos. ${ }^{43}$ Therefore, one has to use nonparametric techniques to test for chaos and construct theoretical models to demonstrate applicability of chaos theory to a setting. In conclusion, although the TAR models can be estimated and loosely identified for agricultural models, they suffer from problems of indentification and variable selection. Therefore, in order to circumvent these problems it was determined that neural network models would allow a richer modeling

43 Testing for chaos in this fashion (i.e. parametric specifications) has not been developed to the point where applied studies can utilize the results. 
environment and would include both the threshold and exponential autoregressive models in their specification, something attempted after testing for chaos. 
CHAPTER 5: METHODOLOGICAL TESTING FOR DYNAMICAL BEHAVIOR IN

\section{EMPIRICAL TIME SERIES}

He who knows not and knows not that he knows not is a fool.

Shun him.

He who knows not and knows that he knows not is a child.

Teach him.

He who knows and knows not that he knows is asleep.

Wake him.

He who knows and knows that he knows is a wise man.

Follow him.

Solomon Ibn Gabriol, Choice of Pearls

\section{Introduction}

Based on the results of the previous chapters, proper classification of the attractors has been demonstrated to be of prime importance if the interest is in agricultural price forecasting. Whether it is Cobwebian or chaotic, one must know the nature of the convergence, periodic or aperiodic. This classification schema from a philosophical perspective is that the economist can view agricultural price behavior as the outcome of

- a deterministic linear system with significant stochastic forces,

- a nonlinear system with insignificant stochastic forces or

- a nonlinear structure with significant stochastic shocks.

Each of the three conditions is complicated by the fact that our statistical measures focus on the following classifications:

- Stochastic,

- Periodic Deterministic, and

- Chaotic or Aperiodic Deterministic.

The mention of "white chaos" in the preceeding chapter demonstrates the difficulty of this attempt to delineate these two non-disparate classifications for the attractor. Therefore, as pointed out in Lui et al. (1992), most empirical treatments of this topic have focused on the first 
philosophical assumption and have invoked the three statistical classifications. Therefore, the treatment here is to make a similar philosophical assumption, while at the same time being cognizant of the restrictive nature of the study in this regard.

In this chapter, the approach is based upon the two "flow-charts" presented in Chapter One. The approach is to provide the test details utilized in the next Chapter on the empirical application of the fore-mentioned ideas. For the purpose of clarity and coherence each test is described and any important caveats disclosed. However, an in-depth investigation is not presented for those tests that are of common usage in the empirical literature. ${ }^{44}$

As a quick review of the procedure, the approach is as follows: In order to ascertain whether or not the attractor is fractal, several steps as outlined in Figure 1 must be performed before we can explicitly test for chaos. First, the attention is directed at the methods for obtaining the attractor's dimension.

\section{Measuring the Attractor}

As mentioned previously, a dynamical system can be stated as a map or a flow. In either case, an orbit or path of the dynamical system is represented by a sequence, $\left\{\mathrm{x}_{1}, \mathrm{x}_{2}, \ldots,\right\}$ which can be either scalars or vectors. If $\mathrm{x}$ is a scalar as is our case here, then the Takens extension to the Whitney Embedding theorem is used to represent one component of the vector at each time $\mathrm{t}$ to represent the flow of the dynamical system. That is to say, that the observation of one component over time can provide us with many of the topological and dynamical properties of the system.

\footnotetext{
${ }^{44}$ Note that the test specifics with respect to empirical implementation are presented in Appendix A.
} 
Dynamical orbits have dynamical properties and attractors have topological properties. Although the concept of dimension is a topological concept, some dimensions have measuretheoretic components. Ramsey and Yaun (1989) state that dimension concepts include:

- The amount of information needed to specify the position of a point on an attractor;

- The lower bound on the number of essential variables that are needed to model the attractor or rather the dynamical system within the attractor;

- The relative density of the points on the attractor.

Unfortunately, there are many definitions of dimension in the nonlinear dynamical literature. In most cases, dimensionality is an Euclidean construct and refers to integral measures which represent "degrees of freedom" or the number of axes needed to represent an attractor.

There are two different approaches for determining the attractor's dimension: the metric and probabilistic. Metric dimensions which include the capacity and Hausdorff dimension are independent of the number of times or frequency with which points visit different neighborhoods of a set represented by the attractor. In other words, all neighborhoods contribute equally. The term neighborhood in this context means that around a point, for example $\mathrm{x}_{0}$, there exists a neighborhood around $\mathrm{x}_{0}$ say $\left(\mathrm{x}_{0}-\delta, \mathrm{x}_{0}+\delta\right)$ where $\delta$ is some arbitrary constant. If the concern is not with the number of times a trajectory enters into this neighborhood then it is called a metric dimension.

Otherwise, a probabilistic dimension such as the information, pointwise and correlation dimension is concerned with the relative frequency for which trajectories enter into these neighborhoods. This relative frequency gives some indication of the degree of correlations 
between points on the attractor. Before the development of each measure, a state or phase space in which to model the dynamics is needed.

\section{Phase Space Construction}

Poincare was among the first to recognize that much could be learned about the dynamical behavior of a dissipative system by examining the system's trajectories in a multi-dimensional phase space in which a single point characterizes the entire system at any given point in time. The set of phase space trajectories for all possible initial conditions forms a phase portrait of the system.

This multi-dimensional phase space can be constructed from the information contained in a single univariate time series. For example, suppose that we have $\mathrm{T}$ observations on an economic variable over time, denoted as $\mathrm{x}_{\mathrm{t}}$. A m-dimensional phase portrait can be constructed by an "embedding" of $\mathrm{x}_{\mathrm{t}}$ in a $\mathrm{n}$-dimensional vector space. This construction is made possible by examining the lags $\mathrm{j}$ of $\mathrm{x}_{\mathrm{t}}$, (i.e. $\left.\mathrm{x}_{\mathrm{t}}, \mathrm{x}_{\mathrm{t}-1}, \ldots, \mathrm{x}_{\mathrm{t}-\mathrm{j}}\right)$, and formulating a vector

$$
\mathrm{X}_{\mathrm{t}}=\left(\mathrm{x}_{\mathrm{t}}, \mathrm{X}_{\mathrm{t}+1}, \ldots, \mathrm{x}_{\mathrm{t}+\mathrm{m}-1}\right)^{\prime} \quad \mathrm{t}=1, \ldots, \mathrm{T}-(\mathrm{m}-1)
$$

where $\mathrm{T}$ is the total number of observations and $\mathrm{m}$ is the choice of the embedding dimension. For example, suppose that $\mathrm{x}_{\mathrm{t}}$ contains six observations; $\mathrm{x}_{1}, \mathrm{x}_{2}, \mathrm{x}_{3}, \mathrm{x}_{4}, \mathrm{x}_{5}, \mathrm{x}_{6}$ and for each value of $\mathrm{m}$, the following column vectors are: 


$$
\begin{array}{llll}
\mathrm{X}_{1} & \mathrm{X}_{2} & \mathrm{X}_{3} \\
\mathrm{~m}=1 & \mathrm{~m}=2 & \mathrm{~m}=3
\end{array}
$$

$\mathrm{X}(1)=\mathrm{x}_{1} \quad \mathrm{X}_{1}, \mathrm{X}_{2} \quad \mathrm{x}_{1}, \mathrm{X}_{2}, \mathrm{X}_{3}$

$\mathrm{X}(2)=\mathrm{x}_{2} \quad \mathrm{x}_{2}, \mathrm{x}_{3} \quad \mathrm{x}_{2}, \mathrm{x}_{3}, \mathrm{x}_{4}$

$\mathrm{X}(3)=\mathrm{X}_{3} \quad \mathrm{X}_{3}, \mathrm{X}_{4} \quad \mathrm{X}_{3}, \mathrm{X}_{4}, \mathrm{X}_{5}$

$\mathrm{X}(4)=\mathrm{X}_{4} \quad \mathrm{X}_{4}, \mathrm{X}_{5} \quad \mathrm{X}_{4}, \mathrm{X}_{5}, \mathrm{X}_{6}$

$\mathrm{X}(5)=\mathrm{X}_{5} \quad \mathrm{X}_{5}, \mathrm{X}_{6}$

$\mathrm{X}(6)=\mathrm{X}_{6}$

These vectors can be used to construct the phase portraits mentioned above. For example, $\mathrm{X}_{2}$ consists of 2-dimensional vectors where $\mathrm{x}_{\mathrm{t}}$ would be placed on the horizontal axis and $\mathrm{x}_{\mathrm{t}+1}$ would be placed on the vertical axis. Similarly a 3-dimensional phase portrait can be examined through the vector $X_{3}$. As a result, the properties of an attractor in $n$-space can be examined by embedding the univariate time series in $m$-space as long as $m \leq 2 n+1$. Once the embedding is performed, then the topological measures can be estimated.

\section{Dimension Measures}

Hausdorff (1919) introduced the idea of a non-integer value for dimension. Bai-Lin (1982) gives a very clear example of Hausdorff's idea, "if we look at a cube, for example, and double its linear size in each of the 3 spatial directions. The volume of this cube is eight times larger than the original one, $2^{3}=8$. Extending this concept to any dimension $\mathrm{D}$, and increasing its linear size in each spatial direction $\mathrm{L}$ times, the volume would increase to $\mathrm{k}=\mathrm{L}^{\mathrm{D}}$ times the original. Inverting this expression, a new definition of dimension surfaces;

$$
\mathrm{D}_{\mathrm{H}}=\ln \mathrm{k} / \ln \mathrm{L}
$$


where $\mathrm{D}_{\mathrm{H}}$ can have non-integer values. For example, if the object is a one dimensional line, $\mathrm{D}=1$ and the length is increased 5 times, $\mathrm{L}=5$, then $\mathrm{k}=5$ and $\mathrm{D}_{\mathrm{H}}=1$. Consider another example, suppose that for the interval $(0,1)$ an object can be constructed by deleting the middle one third section. The interval is now $(1 / 3,2 / 3)$ and we can continue this process of middle deletion, again and again. The dimension of the remaining set of points can be obtained by (5.1). If these points are in the interval $(0,1 / 3)$, increasing the linear size by a factor of $\mathrm{L}=3$ would result in 2 copies, $\mathrm{k}=2$, of this continuing middle deletion, and therefore leads to

$$
\mathrm{D}_{\mathrm{H}}=\ln 2 / \ln 3=0.6309 \ldots
$$

This geometrical object is known in the mathematical literature as a Cantor set. The important result of this application lies in the ability of this method to measure the dimension of a set of points.

Empirical estimation of the Hausdorff dimension involves a box counting algorithm. This box counting algorithm rests on the premise that the attractor's region of a phase space can be divided into small boxes or cells of a linear size, $\varepsilon$. Once this is accomplished then the number of boxes $\mathrm{N}(\varepsilon)$ that contain at least one point of the trajectory embedded in the phase space is counted. Since the Hausdorff measure is a metric measure, we are not concerned with the number of times a trajectory enters the cell just whether it enters once. Similarly, the other metric measure called the Kolmogorov capacity dimension can be obtained by taking the limit

$$
\mathrm{D}_{\mathrm{c}}=\lim _{\varepsilon-\infty \infty} \ln \mathrm{N}(\varepsilon) / \ln (1 / \varepsilon)
$$

where $\mathrm{N}(\varepsilon)$ is the minimum number of e diameter cubes needed to cover the attractor. Since analytical derivation of (5.5) is intractable, empirical practice dictates that $D_{c}$ be obtained from the slope of the plot of $\ln N(\varepsilon)$ vs. ln $1 / \varepsilon$. The capacity dimension is based on the scaling factor $\varepsilon$ 
where $\varepsilon$ is a variable parameter that determines the degree of resolution for the box counting procedure. The scaling factor, $\varepsilon$, examines the degree of self-similarity, that is, the constant rate at which a new structure surfaces. The Hausdorff dimension is defined with respect to the concept of the Hausdorff measure, which in turn is a generalization of Lebesque measure. The Hausdorff dimension will be non-integer when the attractor is strange and has the Cantor set properties.

The problem with metric measures is that they treat all parts of the attractor equally, regardless of the frequency with which an orbit visits various parts of the attractor. Two measures that incorporate the relative frequency of visit by the orbit are the information dimension and the pointwise dimension.

The information dimension extends the metric context of the Hausdorff or capacity dimension to the probabilistic context by recording the number of times a trajectory passes through a given box or cell $\mathrm{N}(\varepsilon)$. In the metric context, even if a trajectory enters the box, $\mathrm{N}(\varepsilon)$, twenty times it is only counted as once. The probabilistic context records the frequency with which a trajectory enters the box. If the ith box is visited with a probability $\mathrm{P}_{\mathrm{i}}$, then (5.5) is re-defined as:

$$
\mathrm{D}_{\mathrm{I}}=\lim _{\varepsilon->\infty} \mathrm{I}(\varepsilon) / \ln (1 / \varepsilon)
$$

where $\mathrm{I}(\varepsilon)$ is Shannon's Information measure and is formally defined by

$$
\mathrm{I}(\varepsilon)=-\Sigma_{\mathrm{i}} \mathrm{P}_{\mathrm{i}} \ln \mathrm{P}_{\mathrm{i}} \quad \mathrm{i}=1, \ldots, \mathrm{N}(\varepsilon)
$$

and $\mathrm{N}(\varepsilon)$ is the same measure and represents total number of boxes covering the attractor visited. $\mathrm{P}_{\mathrm{i}}$ is the relative frequency of occurrence of points on the orbit line in the ith cell. If all the coverings are equally likely, $\mathrm{P}_{\mathrm{i}}=\mathrm{N}(\varepsilon)-1$, then $\mathrm{I}(\varepsilon)=\ln \mathrm{N}(\varepsilon)$ and $\mathrm{D}_{\mathrm{I}}=\mathrm{D}_{\mathrm{C}}$. Most often, $\mathrm{D}_{\mathrm{I}} \leq \mathrm{D}_{\mathrm{C}}$. 
The Pointwise dimension, $\mathrm{D}_{\mathrm{p}}$, is defined by $\mu(\mathrm{B} \varepsilon)$ as the probability measure of a "ball" of radius $\varepsilon ; \mathrm{B} \varepsilon(\mathrm{x})$ is a hypersphere of radius $\varepsilon$ centered at $\mathrm{X}$. The Pointwise dimension at $\mathrm{x}$ is then

$$
\mathrm{D}_{\mathrm{p}}=\lim _{\varepsilon \rightarrow 0} \ln \mu(\mathrm{B} \varepsilon(\mathrm{x})) / \ln (\varepsilon)
$$

If $D_{p}(x)$ is independent of $x$ for almost all $x$ on the attractor, then the common value of $D_{p}(x)$ is the pointwise dimension of the attractor. The Pointwise dimension measures the relative rate of scaling of the probability measure of a ball of radius $\varepsilon$ as the diameter approaches zero. A Pointwise dimension is that measure which uses the Grassberger-Procaccia procedure. As such, this procedure has been referred to as a "correlation dimension" and has become the standard by which dimension estimates of empirical times have been attained.

The correlation dimension, as a probabilistic measure, estimates the correlations between points on an attractor. The correlation exponent $\left(\mathrm{d}_{\mathrm{c}}\right)$ represents a measure of the minimum number of essential variables (degrees of freedom) required to model the dynamics of the attractor. $^{45}$ Correlation exponent estimation is based on the correlation between the elements of the time series that are "embedded" in a higher dimensional space. Takens (1980) suggested that a phase space could be created with $\mathrm{m}$-dimensional vectors from an univariate time series $\mathrm{x}(\mathrm{t})$. These vectors are initially equally spaced, as indexed by some lag parameter, $\gamma$. The value of this lag parameter is assumed to be known and is a function of the sampling interval for the empirical data. Typically, this takes the value one. The choice of $\mathrm{m}$ for the dimensionality of the vectors is subjective since it precludes the knowledge of the unknown attractor's dimension. For example,

45 An example of an attractor would be a stable fixed point where all nearby initial points are attracted to the fixed point. Another type of attractor would be that of a limit cycle. 
it involves assuming $\mathrm{m}$ is greater than the unknown attractor's dimension $\mathrm{d}_{\mathrm{c}}$. In order to be able to reconstruct the attractor for the time series, Takens (1980) suggests a lower bound restriction on $\mathrm{m}$, specifically, $\mathrm{m}>2 \mathrm{~d}_{\mathrm{c}}+1$.

Suppose that an attractor is one-dimensional. This implies that we can examine the local structure of the attractor by examining the correlation between the vectors $\mathrm{x}_{\mathrm{i}}{ }^{* 2}$. The correlation can be examined through the use of a correlation "integral". This correlation integral examines the distance between points in this two dimensional space through the use of the following expression

$$
\mathrm{C}(\mathrm{m}, \mathrm{T}, \varepsilon)=\left(2 / \mathrm{T}_{\mathrm{m}}\left(\mathrm{T}_{\mathrm{m}}-1\right)\right)\left[\Sigma_{\mathrm{t}<\mathrm{s}} \mathrm{I}\left(\varepsilon ; \mathrm{x}^{*} \mathrm{~m}(\mathrm{t}), \mathrm{x}^{*} \mathrm{~m}(\mathrm{~s})\right)\right]
$$

Here $\mathrm{T}_{\mathrm{m}}=\mathrm{T}-\mathrm{m}+1 ; \mathrm{I}\left(\varepsilon ; \mathrm{x}^{*} \mathrm{~m}(\mathrm{t}), \mathrm{x}^{*} \mathrm{~m}(\mathrm{~s})\right)$ constitutes an indicator function that equals one, if $\| \mathrm{x}^{*{ }^{*} \mathrm{t}}(\mathrm{t})-$ $\mathrm{x}^{* \mathrm{~m}}(\mathrm{~s}) \|<\varepsilon$, or that equals zero otherwise. The metric used is the sup norm. In the 2-dimensional example mentioned, the indicator function examines all pairs of vectors $x^{* 2}(t)$ and $x^{* 2}(s)$ while $\mathrm{t}<\mathrm{s}$.

This maximum is then compared to the value of $\varepsilon$. This value of epsilon represents the fluctuating range of the attractor. If the max < epsilon, then the pair is counted, otherwise it is not. As we vary $t$ and $\mathrm{s}$, we count the number of pairs that satisfy this condition. This total is then divided by the total number of pairs to obtain the fraction of pairs that are within distance $\mathrm{e}$ of each other. If the value of epsilon is chosen such that that all pairs satisfy the condition, then $\mathrm{C}(\mathrm{m}, \mathrm{T}, \varepsilon)=1$. Of course, if epsilon is chosen such that the condition is never satisfied, then $\mathrm{C}(\mathrm{m}, \mathrm{T}, \varepsilon)=0$. Thus, the correlation can be thought of as a probability measure for spatial correlation.

The definition of the correlation exponent follows from the above 


$$
d_{c} \approx \lim _{\varepsilon \rightarrow 0} \lim _{t \rightarrow \infty} \ln C(m, T, \varepsilon) / \ln \varepsilon
$$

One can make the above approximation into an equality by varying the estimates of dimension and by employing a sufficiently large number of $\varepsilon$ values which satisfy the condition $\mathrm{C}(\mathrm{m}, \mathrm{T}, \varepsilon)>$ 0. Grassberger and Procaccia (1983) estimate $d_{c}$ directly from

$$
\ln \mathrm{C}(\mathrm{m}, \mathrm{T}, \varepsilon)=\ln \mathrm{k}+\mathrm{d}_{\mathrm{c}} \ln \varepsilon+\mathrm{v}
$$

where $\mathrm{k}$ is a constant and $\mathrm{v}$ is white noise. However, Caswell and Yorke (1986) and Osborne and Provenzale (1989) suggest that $d_{c}$ may be biased. Ramsey and Yaun (1989) have found the direction of the bias to be downward in estimates of correlation exponents of white noise, and upward for low-dimensional attractors. In addition, attempts to identify a linear portion of the plot of $\ln C(m, T, \varepsilon)$ against $\ln \varepsilon$ are difficult because of the existence of noise in the data. Usually, the estimation of (5.11) involves ordinary least squares; however, due to the fact that the correlation integral is a function of $\varepsilon$, serial correlation is considered to be a problem.

As an alternative to the regression procedure, Brock and Baek (1991) define a point estimator as an approximation to the above mentioned elasticity. Their estimator is define as

$$
d_{m}(\varepsilon, \Delta \varepsilon, T)=\frac{\log C(\varepsilon+\Delta \varepsilon, m, T)-\log C(\varepsilon, m, T)}{\log (\varepsilon+\Delta \varepsilon)-\log (\varepsilon)}
$$

Brock and Baek (1991) define this to be distributed asymptotically normal with the variance given by the estimator 


$$
\begin{aligned}
& V D_{m}=4 \gamma^{2}\left[A^{m}+B^{m}-2 C^{m}+2 \sum_{j=1}^{m-1} A^{m-j}+B^{m-j}-2 C^{m-j}\right. \\
& \text { where } \\
& \gamma=\frac{1}{\log (\varepsilon+\Delta \varepsilon)-\log (\varepsilon)} \\
& \begin{array}{l}
A=K(\varepsilon+\Delta \varepsilon) / C(\varepsilon+\Delta \varepsilon)^{2} \\
B=K(\varepsilon) / C(\varepsilon)^{2} \\
C=W(\varepsilon+\Delta \varepsilon, \varepsilon) /(C(\varepsilon+\Delta \varepsilon) C(\varepsilon))
\end{array}
\end{aligned}
$$

Equation (5.13) can be used to construct a hypothesis test concerning the dimension of the time series. For example, Scheinkman and LeBaron (1989a) produced a point estimate of about 6 for the correlation dimension of stock returns that were significant as an outcome of a hypothesis testing procedure.

If the estimate of the correlation exponent is equal to the embedding dimension, $\mathrm{m}$, for each $m$ chosen in $\log \mathrm{C}(\mathrm{m}, \varepsilon, \mathrm{T})$, then the data are interpreted to be independent. Brock, Hsieh and Lebarron (1991) point out that the BDS test that is also based on the correlation integral gives the same result indirectly. Since work involving the correlation integral is based on theoretical asymptotic properties, empirical data sets may not meet this requirement. For example, Frank and Stengos $(1988,1989)$ along with Ramsey and Yuan (1989) point out that for finite data sets the correlation dimension estimate my be substantially below $\mathrm{m}$ and may increase with $\mathrm{m}$ at a much slower rate than one to one. As a result, the slope estimate of the correlation exponent may "saturate" or appear to converge to a constant over increasing values of $\mathrm{m}$. In this case, one can 
obtain a crude estimate of the lower bound on the number of variables or degrees of freedom needed to explain the process $x(t){ }^{46}$

The measures to this point have concentrated on estimating the dimension of the system's attractor. The next two measures, both the Kolmogorov entropy and Lyapunov exponents, inform us if the time series is indeed chaotic.

Since these two procedures both check for the presence of a deterministic structure in the data, Brock and Sayers (1988) suggest that a control sequence is used for white noise to confirm the conclusion of determinism. Their methodology is to generate a sequence of white noise data with the same mean and variance as the data under investigation. Remember that in the case of the correlation dimension, a time series that possesses a deterministic structure will have an $\alpha^{*}$ for the saturation embedding dimension, $\mathrm{m}^{*}$. Theoretically, in the presence of large samples, $\alpha^{*}$ will not exist for white noise; however, it may be that this will not be true for small data sets. Therefore, the control white noise data could possible posses a $\alpha^{* *}$. If this value, $\alpha^{* *}$, is greater than $\alpha^{*}$ from the data in question, then the data has a deterministic structure. If these values are not significantly different, then the conclusion is rejected. Since this test relates to equality of means from an unknown probability distribution, nonparametric procedures could be invoked. Similarly, the same procedure can be performed for the Kolmogorov entropy by obtaining the value $\mathrm{K}^{*}$ for white noise data and performing a significance test.

In order to be sure that the detected structure is nonlinear rather that linear, Brock's (1987) residual test is employed. Brock's residual test theorem states that, given a set of regularity conditions, the GP correlation dimension and the largest Lyapunov exponent of $\mathrm{x}(\mathrm{t})$ 
and the residuals $e(t)$ are the same. This result suggests that the tools for analyzing nonlinear dynamical behavior can be applied to the original data $\mathrm{x}(\mathrm{t})$ as well as the associated residuals from standard time series models. Thus, the approach can be to use linear AR models, ARCHGARCH models or a neural network approach. Because of problems with the robustness of the correlation dimension technique, one must be cautious in the number of terms utilized in the autoregression. Therefore, a detailed examination is given with respect to the autoregression performed as apart of this test.

\section{Testing For Stationarity}

Stationarity is the first fundamental statistical property tested for in time series analysis, because most statistical models require that the underlying generating processes be stationary. If a process is not stationary (nonstationary), then hopefully a transformation of the data can be performed in such a way as to render that process weakly stationary. Since most real world data are nonstationary, performing transformations and testing for stationarity should coincide. Standard tests of Dickey-Fuller (1979) are employed here.

\section{Testing For Normality}

The familiar concept of a normal distribution can be combined with independence to provide insights into methods of specifiying a time series model. If a time series has been transformed to achieve stationarity and if that series passes the test of normality, then it is possible to specify a linear model to describe the behavior of that time series. The tests provided

${ }^{46}$ Smith (1991) developed the "independent distance hypothesis" in his binomial estimator which is used as an alternative estimator of the correlation dimension. 
here prepare the way for this aspect of model specification. Although a number of normality tests exist, only those considered more relevant are presented. For a survey of other available tests, please refer to Conover (1980) and D'Agostino and Stephens (1986).

As a result of previous research on economic price series, Mandelbrot (1963) and Fama (1963) hypothesized that the distribution of log price changes is best fitted by stable distribution laws. The ability of a time series to be represented by normality as stated above also has implications with respect to stable Paretian distributions. Stable Paretian distributions are used to describe leptokurtic distributions. For example, according to the Mandlebrot-Fama hypothesis, the distributions of returns is stable Paretian with a characteristic exponent $1<\alpha<2$. This hypothesizes suggests that the distributions of returns have an infinite theoretical variance and that the distributions are stable. That is, they may be defined by the log characteristic function:

$$
\log \varphi(t)=i \delta t-\gamma|t|^{\alpha}
$$

where $\delta, \gamma \geq 0$ and $0<\alpha \leq 2$ are real numbers and $\mathrm{i}$ is the imaginary unit. Symmetric stable Paretian distributions are completely described by their parameters: $\delta, \gamma$ and $\alpha$. For example,

- $\delta$ is the location parameter.

- $\gamma$ is equal to $\mathrm{c} \alpha$, where $\mathrm{c}$ is the scale parameter as alternative to the $\sigma$ for normal distributions and $\alpha$ is the characteristic exponent. This parameter determines the shape of the distribution. When $\mathrm{z}=2$, the distribution is normal. When $\alpha$ is smaller than 2 , the number of observations near the mean and at both extremes of the distribution will be higher than is usual with normal distributions.

In order to determine the value of $\alpha$ Fama and Roll (1968) developed the following procedure. The concept involves that for every distribution, there is a unique $\alpha$ which can be 
determined from close observation of the tails of the distribution. For example, the thickness of the tails is indicative of the leptokurtosis.

The procedure is as follows: First, arrange the data from lowest to the highest value. Then the 95th fractile observation should be standardized:

$$
z=\frac{x(\alpha, 0.95)-\delta}{c}
$$

where $\mathrm{x}(\alpha, 0.95)$ is the 95 th fractile observation from the ranked series. Both values have to be estimated. The parameter c can be estimated from equation (5.3), Fama and Roll (1968) yields an estimated error of less that $0.4 \%$.

$$
c=\frac{x(\alpha, 0.72)-x(\alpha, 0.28)}{1.654}
$$

The calculated standardized value, $\mathrm{z}$, can be compared with the $\mathrm{z}$ values from known Paretian distributions. For this, Fama and Roll (1968) provide a table of standard z-values for 12 distributions with different $\alpha$-values. The $\alpha$-value of the matching standard distributions is also the $\alpha$-value of the observed distribution.

\section{Testing For Independence}

Time series that are independent can be thought of as non-correlated. From a definition standpoint, if the product of the marginal distributions of two independent random variables is the same as their joint distribution, these two random variables are statistically independent. Our understanding of independence can be coupled with that of normality as part of the time series model specification process mentioned in Chapter 3. Tests for independence can be applied to see if the time series, $x(t)$, displays independence. For example, if the time series $x(t)$ is 
independent, then the process is completely random, and no deterministic model can be constructed. Tests for normality can also be applied to the stochastic error term, e(t), from the equation for $\mathrm{x}(\mathrm{t})$ in order to achieve a better interpretation of model specification.

Consider also the possibility that a time series can appear to be random yet also display regularities (deterministic components). Such behavior has been attributed to time series that are chaotic. For a review of the definitions, conditions and tests for chaos, refer to Benhabib and Nishimura (1979), Gleick (1987) or Baumol and Benhabib (1989).

Among the independence tests described in this chapter, the first four are the more traditional: Ljung-Box and Box-Pierce, Turning Points, Runs Analysis, and RV-Von Neuman's Ratio. One of the more recent tests is that of Brock, Dechert and Scheinkman (1986).

The literature on testing for chaos contains the Brock, Dechert, and Scheinkman (1986) statistic (BDS), a non-parametric test, which tests the null hypothesis of independence for a time series $\mathrm{x}(\mathrm{t})$ by utilizing the concept of spatial correlation. To examine this "spatial" correlation, the time series, $\mathrm{x}(\mathrm{t})$, must be embedded in $m$-space by constructing the following vector,

$$
x^{m}(t)=[x(t), \ldots, x(t-m+1)] \quad t=1,2, \ldots, T-m+1
$$

Takens (1980) suggested this concept of embedding which can be illustrated for the data set $\mathrm{x}(\mathrm{t})$ where $\mathrm{t}=1,2, . .5$ and $\mathrm{m}=3$.

$$
\begin{aligned}
x^{3}(1) & =[x(1) x(2) x(3)] \\
x^{3}(2) & =[x(2) x(3) x(4)] \\
x^{3}(3) & =[x(3) x(4) x(5)]
\end{aligned}
$$

In this case the "embedding" operation creates three new three-dimensional vectors $\mathrm{x}^{3}(1), \mathrm{x}^{3}(2)$ and $x^{3}(3)$. Because it is required that the vectors all be of equal length with this method, $m-1$ 
data points are lost as in the above illustration. Thus, embedding enables one to take a univariate variable and "embed" it into a higher dimensional space. The choice of $\mathrm{m}$ for the dimensionality of the vectors is subjective. The motivation behind this is to examine the correlation in a spatial context.

The dependence of $\mathrm{x}(\mathrm{t})$ is examined through the concept of the correlation integral, a measure which examines the distances between points in our 3-dimensional example. For each embedding dimension, $\mathrm{m}$, and choice of epsilon, e, the correlation integral is defined by

$$
\mathrm{C}(\varepsilon, \mathrm{m}, \mathrm{T})=\left(\mathrm{T}_{\mathrm{m}}\left(\mathrm{T}_{\mathrm{m}}-1\right)\right)^{-1} \Sigma_{\mathrm{t} \neq \mathrm{s}} \mathrm{I}\left(\mathrm{x}^{\mathrm{m}}(\mathrm{t}), \mathrm{x}^{\mathrm{m}}(\mathrm{s}) ; \varepsilon\right)
$$

where $T_{m}=T-m+1, t$ and $s$ both range from 1 to $T-m+1$ in the summation and are restricted such that $\mathrm{t} \neq \mathrm{s}$. The indicator function in (5.19) is

$$
I\left(x^{m}(t), x^{m}(s) ; \varepsilon\right)=\begin{array}{ll}
1 & \text { if }\left\|x^{m}(t)-x^{m}(s)\right\| \leq \varepsilon \\
0 & \text { otherwise }
\end{array}
$$

The term in absolute bars is called a metric or norm where the maximum norm is given by $\|x\|=$ $\max \left|x_{i}\right|$. Thus, the correlation integral will measure the fraction of total pairs of $\left(x^{m}(t), x^{m}(s)\right)$ for which the distance between $\mathrm{x}^{\mathrm{m}}(\mathrm{t})$ and $\mathrm{x}^{\mathrm{m}}(\mathrm{s})$ is no more than epsilon, $\varepsilon$. For example, with a chosen embedding dimension of 3 , the distance between $x^{3}(1)=[x(1), x(2), x(3)]$ and $x^{3}(3)=$ $[x(3), x(4), x(5)]$ is computed by $x^{3}(1)-x^{3}(3)=[x(1)-x(3), x(2)-x(4), x(3)-x(5)]$. One then chooses the maximum element of this resulting vector. This maximum element is then compared to the chosen value of $\varepsilon$.

If this maximum $>\varepsilon$, then the pair is counted; otherwise it is not. As we vary $t$ and $s$, we count the number of pairs that satisfy the condition. Then we divide this total number of pairs that satisfy the condition by the total number of possible pairs. Caution must be exercised not to 
count any pair twice. This expression then yields the fraction of pairs that are within e distance of each other. If the value of e is chosen such that all pairs satisfy the condition then $\mathrm{C}(\mathrm{m}, \mathrm{T}, \varepsilon)=1$. Of course, if e is chosen such that the condition is never satisfied, then $\mathrm{C}(\mathrm{m}, \mathrm{T}, \varepsilon)=0$. This is why the correlation integral is interpreted as a measure of spatial correlation.

Brock and Baek (1991) have demonstrated that under the null hypothesis of independence, the BDS test statistic will be asymptotically standard normally distributed

$$
\mathrm{W}(\varepsilon, \mathrm{m}, \mathrm{T})=\left\{\mathrm{T}^{1 / 2}\left[\mathrm{C}(\varepsilon, \mathrm{m}, \mathrm{T})-\left[\mathrm{C}(\varepsilon, 1, \mathrm{~T})^{\mathrm{m}}\right]\right\} / \mathrm{V}^{1 / 2}\right.
$$

where the variance is defined by

$$
\mathrm{V}=4\left[\mathrm{~K}(\varepsilon)^{\mathrm{m}}+2\left(\Sigma_{\mathrm{i}} \mathrm{K}(\varepsilon)^{\mathrm{m}-\mathrm{i}} \mathrm{C}(\varepsilon)^{2 \mathrm{i}}+(\mathrm{m}-1)^{2} \mathrm{C}(\varepsilon)^{2 \mathrm{~m}}-\mathrm{m}^{2} \mathrm{~K}(\varepsilon) \mathrm{C}(\varepsilon)^{2 \mathrm{~m}-2}\right]\right.
$$

where $\sum_{i}$ for $\mathrm{i}=1$ to $\mathrm{m}-1$. Here the indicator functions $\mathrm{C}(\varepsilon)=\mathrm{EI}(\mathrm{x}(\mathrm{i}), \mathrm{x}(\mathrm{j}) ; \varepsilon)$ and $\mathrm{K}(\varepsilon)=$ $\mathrm{EI}(\mathrm{x}(\mathrm{i}), \mathrm{x}(\mathrm{j}) ; \varepsilon) \mathrm{I}(\mathrm{x}(\mathrm{j}), \mathrm{x}(\mathrm{k}) ; \varepsilon)$ are defined similarly.

Notice that the statistic is a function of two unknowns, the embedding dimension $\mathrm{m}$ and the epsilon, $\varepsilon$. An important relation exists between the choice of $\varepsilon$ and $\mathrm{m}$, and the small sample properties of the BDS statistic. For a given $\mathrm{m}, \varepsilon$ cannot be too small because $\mathrm{C}(\varepsilon, \mathrm{T})$ will capture too few points; similarly $\varepsilon$ cannot be too large, to prevent $\mathrm{C}(\varepsilon, \mathrm{T})$ from capturing too many points. Often in empirical practice, $\varepsilon$ is set in terms of the standard deviation of the data, with $\varepsilon=\sigma$ implying that it is one standard deviation of the data. Some researchers transform the data, $x(t)$, to the unit interval, $[0,1]$, and set epsilon, $\varepsilon=0.9^{\mathrm{i}}$, where $\mathrm{i}$ is from $1, \ldots, \mathrm{m}-1$. The embedding dimension, $\mathrm{m}$, typically is chosen over the range 1 to 15 . Thus, the BDS test is computed over a grid of epsilons and embedding dimensions. Since many tests are being performed which may give contradictory information, care must be undertaken in interpretation. 
To insure that the BDS test is not merely reflecting linear dependence in the data, one can pre-filter the data. Brock (1987) showed that the asymptotic distribution of the BDS statistic applies to residuals of linear regressions as well as to the original data. Thus, any linear dependence that exists can be removed before applying the test to the data. This amounts to a diagnostic on the residuals, useful for testing the stationary and independent assumptions discussed earlier.

Brock et al. (1991) state that their Monte Carlo simulations demonstrate that the standard normal distribution is a good approximation for samples of more than 500 observations. When the data are normally distributed and are fairly robust to problems of skewness and leptokurtosis, empirical practice often dictates the use of a smaller number of observations. In this case, and when an $\mathrm{ARCH}$ or a GARCH effect is present in the residuals, the asymptotic distribution of the BDS test may not be normal. As was reported in Brock et al. (1991), if the critical values from the standard normal are to be used, the number of observations should be greater than 500; the dimension should be $\mathrm{m} \leq 5$; and epsilon should be between 0.5 and 2 standard deviations of the data.

Lee, White and Granger (1990) have compared the BDS test with other tests of nonlinearity for a wide variety of univariate nonlinear processes. Their results indicated that the test had good power under the nonlinear alternative. This was also echoed in the work done by Hsieh and Lebaron (1991) and Hsieh (1991).

\section{Testing For Linear or Nonlinear Dependence}

If a time series lacks independence, it possesses some form of dependence that can be either simple/complex or weak/strong. In the simplest case this dependence may reflect a linear 
trend; more complex processes may require a nonlinear representation. Even the latter can be simple, such as an exponential. In the past, time series dependence has often been evaluated in the frequency domain using tests of spectral analysis. More recently, this approach has also been extended. For example, by Subba Rao and Gabr (1980) and by Hinich (1982) who emphasize a bispectrum test based on bispectral analysis. In this study, time series dependence has been examined mostly using tests in the time domain. Most of these dependence tests are parametric and are based on what has become known as the Volterra expansion. These tests evaluate linear as compared to nonlinear dependence in a time series; an exception is the Hsieh Test that additionally identifies the actual type of nonlinear dependence. Nonparametric tests such as the neural network test (e.g. Lee, et al., 1993) can also be performed but are not included here.

The premise for these tests does involve the specification of a linear model under the null hypothesis that is then estimated and the residuals computed. Regression techniques are then used to examine the correlation between the residuals and power transformations of the variable in question. This method also enables the BDS test from the previous chapter to be included in tests for nonlinear dependence. For example, if a linear model is the correct specification then the residuals will be independent (e.g. BDS test). As in the previous chapter, not all possible tests of dependence are included here.

\section{Alternative Filtering Methods}

One fundamental problem in econometrics is the selection and specification of functional forms as was discussed and illustrated in Chapter 4. Misspecficiation often results in inconsistent estimates and the associated test statistics are also rendered invalid. In most cases, 
as is here, the true underlying model is never known, and consequently one cannot be sure which functional representation should be utilized, therefore, there will always be the possibility of misspecification. Results based on misspecified models can have serious consequences for policy analysis and forecasting. Therefore, the assumption of the linear GARCH model is relaxed and the analysis is conducted with alternative modeling apporaches.

One approach to overcoming this assumption is to use neural network methods to approximate the function. These methods state that any function can be approximated by the sum of composites of functions accompanied by thresholds. From an abstract econometric point of view, White (1989) showed that neural network methods are simply parametric nonlinear models.

A natural extension of the linear $\mathrm{AR}(\mathrm{p})$ model to the nonlinear case would be the nonlinear autoregressive $(\mathrm{NAR}(\mathrm{p}))$ model

$$
x(t)=h(x(t-1), x(t-2), \ldots, x(t-p))+e(t)
$$

where $\mathrm{h}$ is an unknown smooth function that $\mathrm{E}(\mathrm{e}(\mathrm{t}) \mid \mathrm{x}(\mathrm{t}-1), \mathrm{x}(\mathrm{t}-2), \ldots)=0$, with $\mathrm{e}(\mathrm{t})$ having a finite variance $\sigma^{2}$. Under these conditions the minimum mean square error optimal predictor of $x(t)$ is given by the conditional mean.

Feed-forward networks as NAR models for time series prediction were proposed by Lapedes and Farber (1988). In general, a feedforward network is a nonlinear approximation to thefunction $\mathrm{h}$ is given by

$$
x(t)=\sum_{i=1}^{I} W_{i} f_{i}\left(\sum_{j=1}^{P} w_{i j} x(t-j)+\alpha_{i}\right)
$$

where the function $\mathrm{f}(\mathrm{x})$ is a smooth bounded monotonic function, typically a sigmoid. Other functions for $\mathrm{f}$ include: 
- logistic

- linear

- $\tanh$

- $\tanh 1.5$

- sine

- symmetric logistic

- Gaussian

- Gaussian complement

$$
\begin{aligned}
& \mathrm{f}(\mathrm{x})=1 /(1+\exp (-\mathrm{x})) \\
& \mathrm{f}(\mathrm{x})=\mathrm{x} \\
& \mathrm{f}(\mathrm{x})=\tanh (\mathrm{x}) \\
& \mathrm{f}(\mathrm{x})=\tanh (1.5 \mathrm{x}) \\
& \mathrm{f}(\mathrm{x})=\sin (\mathrm{x}) \\
& \mathrm{f}(\mathrm{x})=1 /(1+\exp (-\mathrm{x}))-1 \\
& \mathrm{f}(\mathrm{x})=\exp \left(-\mathrm{x}^{2}\right) \\
& \mathrm{f}(\mathrm{x})=1-\exp \left(-\mathrm{x}^{2}\right)
\end{aligned}
$$

The parameters $\mathrm{W}_{\mathrm{i}}$ and $\mathrm{w}_{\mathrm{ij}}$ are obtained by minimizing the sum of squared residuals based on the sample of observations. Quite often, this is accomplished by the gradient descent procedure known as "backpropagation" or by other optimization routines.

As an extension to traditional ARMA time series models, recurrent networks are networks that demonstrate feedback. One natural extension to these models is the nonlinear autoregresive moving average model of NARMA(p,q). As an approximation a NARMA(p,q) model can be represented by a recurrent neural net.

$$
x(t)=\sum_{i=1}^{I} W_{i} g_{i}(t) \text { where } g_{i}(t)=f\left(\sum_{j=1}^{P} w_{i j} x(t-j)+v_{i j} x(t-j-1)+\alpha_{i}\right)
$$

The virtual long-term memory remembers the hidden layer, which contains patterns detected in the data of previous patterns. This is the most powerful recurrent network and is commonly referred to as the Jordan-Elman recurrent network.

$$
x(t)=\sum_{i=1}^{I} W_{i} g_{i}(t)
$$

where

$$
g_{i}(t)=f\left(\sum_{j=1}^{P} w_{i j} x(t-j)+\sum_{l=1}^{I} v_{i} \mathrm{~g} g(t-1)+\alpha_{i}\right)
$$

Long-term memory remembers the output previous predicted and transforms (5.26) into

$$
x(t)=\sum_{i=1}^{I} W_{i} g_{i}(t)
$$


where

$$
g_{i}(t)=f\left(\sum_{j=1}^{P} w_{i j} x(t-j)+v_{i j} x(t-1)+\alpha_{i}\right)
$$

A recurrent network works well in the presence of nonstationary data as is the case in this context.

Nonparametric methods e.g. Barnett, Powell and Tauchen, 1990, Hardle 1990, and Silverman 1986) involve estimation of conditional densities and nonlinear regression functions by methods that exploit the maintained hypothesis of differentiability. For example, if the function was determinsitic and chaotic then effective short-run prediction could still occur and be done by estimating the function by a nonparametric regression.

Application of kernel methods and nearest neighbor methods to estimate the regression function (conditional mean) of the variables can be used to determine the correct filtering model. Kernel methods have been extensively used in econometric applications (see Ullah (1988) for a survey). Applications of nearest neighbor methods include the work of Robinson (1987) in a regression context as well as the work of Farmer and Sidorowich (1987, 1988a, 1988b) in a forecasting context.

The nearest neighbor method can be explained in the following way. Embed the time series $\{\mathrm{x}(\mathrm{t})\}$ into a $\mathrm{k}$ component vector $\mathrm{x}^{\mathrm{k}}(\mathrm{t})=(\mathrm{x}(\mathrm{t}), \mathrm{x}(\mathrm{t}-1), \ldots, \mathrm{x}(\mathrm{t}-\mathrm{k}+1))$. In the context of estimating chaotic systems the above $\mathrm{k}$ vectors are known as k-histories. In that context, for $\mathrm{k}$ sufficiently long there is a diffeomorphism between the data generating process which cannot be observed and the observed collection of these k-vectors or k-histories.

For forecasting purposes, one takes the most recent history available and searches over the set of all k-histories to find the K nearest neighbors. For example, if one wants to forecast $\mathrm{x}(\mathrm{t})$ from the information available at $\mathrm{t}-1$, one computes the distances of the vector $\mathrm{x}_{\mathrm{k}}(\mathrm{t}-1)=(\mathrm{x}(\mathrm{t}-$ 
$1), \mathrm{x}(\mathrm{t}-2), \ldots, \mathrm{x}(\mathrm{t}-\mathrm{k}))$ and its $\mathrm{K}$-nearest neighbors to form an alternative estimator of $\mathrm{E}[\mathrm{x}(\mathrm{t}) \mid \mathrm{x}(\mathrm{t}-$ 1), $\mathrm{x}(\mathrm{t}-2), \ldots, \mathrm{x}(\mathrm{t}-\mathrm{k})]$. Typically one uses the Euclidean distance. For a more thorough discussion on weighting functions see Robinson (1987).

The shuffle test is also appropriate, because of the finite nature of the study and the tendency for the idealized behavior of the structure data and the noise to separate. This procedure from Scheinkman and LeBaron (1989) tests the hypothesis that the correlation dimension estimate came from a data series of noise with the same distribution as that exhibited by the actual data.

\section{Wavelet Analysis}

Because of the lack of invariant temporal structure in agricultural prices, it is necessary to utilize tools that are robust to this form of structure. Wavelet analysis provides a method of extracting information that is inherent in a time series that may go undetected by the use of other techniques. For example,

- The time series is mapped to a "wavelet" space which enables one to examine a part of the time series from a variety of amplitude and duration perspectives;

- These features are enhanced in this transformed space and it is determined if they are significant or negligible;

- Finally, the amount of information is the reduced by a variety of statistical techniques. In other words, we can reduce the variance and thereby eliminate to a significant degree the non-stationarity problem. ${ }^{47}$

Wavelet analysis is not of recent mathematical origin, but has become increasing popular in recent years. First applications arised in the areas of seismology (Morlet, 1983) and others have occurred in signal processing (see Kronland-Martinet, Morlet and Grossman, 1987) and

\footnotetext{
${ }^{47}$ While de-noising the data is an important application of the wavelet theory to economics, it is by no means the only application.
} 
numerical analysis (Beylkin, Coifman, and Rokhlin, 1991). Economic applications include the work of Jensen (1994) and Greenblatt (1994). There has been a literature that has been growing rapidly with respect to using wavelets for denoising and smoothing (Donoho, 1992; Donoho, Johnstone, Kerkyacharian, and Picard 1994; Donoha and Johnstone 1994; Donoho and Johnstone 1993). The purpose behind this application is to remove the stochastic elements present in the time series in order to understand the empirical regularities that are present. A signla can them be approximated and the wavelets can preserve the local features (which are discountinuities, turning points, etc.) while still removing noise.

The utlity of wavelet analysis is in its ability to localize processes in terms of their frequency-time and space. Properties of wavelet analysis enable a very fine resolution of a process' behavior at a certain frequency level with a time interval that is function of that frequency. Thus, the time frequency relationship at high frequency levels, the wavelet is tight in shape and is able to focus on short-lived phenomena, while at lower frequencies the wavelt has a broader time window. In other words, wavelets allow simultaneous decomposition of a time series into components (bases) that are localized in both time and frequency. This is quite different than the FFT where the component sine and cosine waves are localized in frequency but totally unlocalized in time.

Generally, in the examination of a time series data set analysis ocurrs in one of two ways. We can examine the data in temporal sequence or we can compute the Fourier transform of the series, $f(\omega)$, to obtain the spectrum of the series. In this form, the frequency context is understandable, but the time content is not, the time content is hidden away in the phase of $f(\omega)$. 
When nonstationarity occurs, we need to have both views of the series. That is to say, it would be useful to have a frequency decomposition of $\mathrm{f}(\mathrm{x}(\mathrm{t}))$ locally in time. Traditional Fourier analysis that is global in its examination of the series, does not have the ability of separating out these local frequency components. Modifications of the Fourier transform, known as windowed Fourier transforms, have been developed to address this issue, with limited success. ${ }^{48}$

There are a wide variety of types of wavelets that have been developed and discussed at length in the literature. For the purposes here, we will concentrate on only one particular family of wavelets, compactly supported orthonormal wavelets (Daubechies, 1988). The rationale behind this selection is that these wavelets have particular desirable properties. One such property is that of being compactly supported, so they can accurately represent local, nonperiodic features with a relatively small number of coefficients. This is direct contrast to Fourier analysis, which needs a large number of coefficients to represent local, non-periodic features. In addition this family of wavelets has that property that does preserve the $\mathrm{L}^{2}$ norm during both decomposition and reconstruction.

Quite often, we perform smoothing low pass filters to remove nonstationary components such as trends. This in effect is computing a moving average of the data. This lowpass filter is known as a scaling function, $\phi(\mathrm{x})$. Convolving the data with $\phi(\mathrm{x})$ gives us an approximation of the original series, except with some (high frequency) detail filtered out.

\footnotetext{
48 Daubechies (1990) for a basic comparsion between the wavelets and the windowed Fourier transforms.
} 
In order to obtain detailed information, then we must pass the data in question through a differencing (high pass) filter. This high pass filter is known as a wavelet $(\psi(\mathrm{x}))$. The wavelet can be defined using the notation of Jensen (1994) as:

$$
\psi_{m, n}(t)=a_{0}^{m / 2} \psi\left(a_{0}^{m}\left(t-n b_{0} a_{0}^{-m}\right)\right)
$$

where $\mathrm{a}_{0}>0$ and $\mathrm{b}_{0}>0$, and $\mathrm{m}$ and $\mathrm{n}$ are respectively the dilation and translation parameters which are elements of $\mathrm{Z}$. The function $\psi(\mathrm{t})$ is often referred to as the mother wavelet function which must satisfy the admissibility condition of $\int \psi(t) d t=0 .{ }^{49}$

Under the above stated conditions, (5.28) becomes:

$$
\psi_{m, n}(t)=2^{m / 2} \psi\left(2^{m} t-n\right)
$$

where the strength of the representation in (5.29) is in the fact that if $\psi(t)$ is localized around zero in time and $\omega_{0}$ in frequency, the dilated and translated wavelets $\psi_{\mathrm{m}, \mathrm{n}}(\mathrm{t})$ will also be well localized around $2^{-m} n$ in time and $2^{m} \omega_{o}$ in frequency. Therefore, by increasing and decreasing $m$ and $n$ the wavelet covers different levels of frequency and is shifted over the time horizon.

Jensen (1994), building on the work of Mallat (1989), demonstrates that we can use multiresolution analysis to decompose the time series $\mathrm{x}(\mathrm{t})$ into an orthonomal basis which is completely characterized by the wavelet coefficents in (5.29). Therefore, for each scale the wavelet coefficents of $\mathrm{x}(\mathrm{t})$ decomposes the signal into a set of independent frequency channels. If we normalize the time series to the unit interval the translation parameter $\mathrm{n}$ is now a function

\footnotetext{
${ }^{49}$ The admissibility condition is a necessary one for it insures that the wavelet possess the properties of smoothness and localization in the frequency and time domain. As part of these requirements, we set the parameters of $a_{0}=2$ and $b_{0}=1$.
} 
of the scaling parameter $m$. Thus, $m=1$ and $n=1$ will cover the entire interval since the wavelet is $\left[(\mathrm{n}-1)^{2}-(\mathrm{m}-1), \mathrm{n}^{2}-(\mathrm{m}-1)\right] .{ }^{50}$

To provide an intuitive framework into the application of wavelet theory in the context of determining the dynamical behavior of agricultural prices, consider the following time series $\mathrm{x}(\mathrm{t})$. We can perform the wavelet transformation on the raw time series $x(t)$ or we can translate the series to the unit interval, $[0,1]$. Next, we can select from a variety of bases to conduct the transformation. For example, if we select the Daubechies 4 basis wavelet and conduct a frequency decomposition based on this wavelet, we can obtain a set of coefficients based on each frequency band. Each frequency band is based on the Nyquist critical frequency or $1 / 2$ the sampling rate of the sampled series. In this case the data is sampled monthly, therefore the transformed $\mathrm{x}(\mathrm{t})$ possess in terms of coefficients represents different frequencies. Each result of seven coefficient series represent the spectral components of $x(t)$. If we summed each of the seven coefficients by frequency then we would obtain $x(t)$. These seven coefficient series represent the multi-resolution frequency analysis. Each of these seven series represents the information content of the frequency bands.

The next step would be to remove those frequencies bands that provide very little information content to the series. The final step is to recover the original time series and that can be accomplished by doing inverse transform. However, we can apply a threshold, i.e., 75\%, which effectively eliminates the smallest $75 \%$ of coefficients. That is, with only $25 \%$ of the coefficients used to retrieve the original series, we obtain a good approximation of original series $x(t)$. This provides a way of removing the noise and helps to uncover the structure that is

\footnotetext{
${ }^{50}$ For a detailed discussion on multi-resolution analysis, I refer to the work by Mallat (1989) and Myer (1990).
} 
inherent in the data. ${ }^{51}$ This procedure is followed in this work we the coefficients are then utilized in a neural network methodology to obtain forecasts of the data series. It is a combination of a filter and outlier detection procedure. That is that the time series is decomposed into component series and then thresholded. The new data series, $x *(t)$ is then forecasted used neural network methods. This step does not need any fundamental assumptions of stationarity and is considered to be more robust then ARCH/GARCH specifications.

\section{Empirical Determination of Chaotic Behavior}

There are various measures that have been proposed for characterizing dynamical systems. The fractal dimension, Kolmogorov entropy, and Lyapunov exponents attempt to measure the structure of periodic orbits.

Fractal estimates attempt to provide evidence for an integer attractor. Some chaotic systems have non-integer dimensions. Kolmogorov entropy attempts to measure the average rate at which information is gained by observing a trajectory to a given precision. Lyapunov exponents measure the average rates at which nearby trajectories separate or converge. Therefore, they are essentially the eigenvalues averaged over the entire attractor. For an attractor to be chaotic, it must have at least one positive Lyapunov exponent.

These measures serve as a tool for supplying evidence but in no way provide a suitable hypothesis testing framework. We will first examine the Kolmogorov entropy since it expands on the concept of the correlation integral discussed above.

\footnotetext{
${ }^{51}$ Like the FFT, the discrete wavelet transform suffers for aliasing effects at the endpoints of the series. In order to overcome the problem, the Daub4 transform was used because of its sharp corners, i.e. discontinuties of the first derivatives, which is a good approximation for agricultural data.
} 
Kolmogorov entropy relates to the correlation dimension by examining the correlation interval over interval changes in the embedding dimension, $\mathrm{m}$. Kolmogorov entropy, $\mathrm{K}_{\mathrm{e}}$, detects the presence of noise, periodic determinism, and non-periodic determinism. For example, $\mathrm{K}_{\mathrm{e}}$ is infinite for white noise, zero for periodic determinism, and positive and finite for non-periodic determinism.

Grassberger and Procaccia (1983) compute a lower bound for $\mathrm{K}_{\mathrm{e}}$ through the following expression

$$
\mathrm{K}(\varepsilon, \mathrm{m})=\ln [\mathrm{C}(\varepsilon, \mathrm{m}) / \mathrm{C}(\varepsilon, \mathrm{m}+1)]
$$

where $C(\varepsilon, m)$ is our correlation integral defined in the previous section and $d_{m}$ is the interval between successive values of $\mathrm{m}$ considered. Once $\mathrm{K}(\varepsilon, \mathrm{m})$ is calculated for various $\mathrm{e}$ in the scaling region for a given $\mathrm{m}$ and then averaged to get $\mathrm{K}(\mathrm{m})$, a limit is taken with respect to $\mathrm{m}->\infty$, denote as $\mathrm{K}^{*}$. This $\mathrm{K}^{*}$ is a lower bound estimate for $\mathrm{K}_{\mathrm{e}}$.

For a finite epsilon, a series is independent and identically distributed if $\mathrm{K}(\varepsilon, \mathrm{m})=-\log$ $(\mathrm{C}(\varepsilon, 1)) \rightarrow \infty$, as $\varepsilon \rightarrow 0$. The standard error of the approximate Kolmogorov entropy $\mathrm{Km}(\varepsilon)-$ $\log (\mathrm{C}(\varepsilon, \mathrm{m}) / \mathrm{C}(\varepsilon, \mathrm{m}+1))$ can be obtained by $\mathrm{U}$ statistics. For example, the variance of $\mathrm{K}^{\mathrm{m}}(\varepsilon)$ is given by $\mathrm{Vk}^{\mathrm{m}}=4\left[\left\{\mathrm{~K}(\varepsilon) / \mathrm{C}(\varepsilon)^{2}\right\}^{\mathrm{m}+1}-\left\{\mathrm{K}(\varepsilon) / \mathrm{C}(\varepsilon)^{2}\right\}^{\mathrm{m}}\right]$.

The Lyapunov dimension is constructed with the generalized eigenvalues that give the average rates of contraction or expansion on an entire attractor. Precisely, one considers small volume elements at various points. But rather than simply determining whether these elements expand or contract, orthogonal directions are defined into which the motion is to be resolved. ${ }^{52}$

\footnotetext{
${ }^{52}$ This is accomplished by employing the Gram-Schmidt reorthonormalization procedure.
} 
To provide an understanding of this concept, consider the example of such a system, the non-linear autoregressive model

$$
x(t)=f(x(t-1), x(t-2), \ldots, x(t-d))+e(t)
$$

with $\mathrm{x}(\mathrm{t})$ in $\mathfrak{R}$ and $\{\mathrm{e}(\mathrm{t})\}$ is a sequence of IID random variables. It is useful to express this system in terms of a state vector $\mathrm{X}(\mathrm{t})=(\mathrm{x}(\mathrm{t}), \mathrm{x}(\mathrm{t}-1), \ldots, \mathrm{x}(\mathrm{t}-\mathrm{d}+1))^{\prime}$, an error vector $\mathrm{e}(\mathrm{t})=$ $(\mathrm{e}(\mathrm{t}), 0,0, \ldots, 0)^{\prime}$ in $\mathfrak{R}^{\mathrm{d}}$, such that there exists a function $\mathrm{F}: \mathfrak{R}^{\mathrm{d}} \rightarrow \mathfrak{R}^{\mathrm{d}}$ such that

$$
X(t)=A X(t-1)+e(t)
$$

where $A$ is a DxD matrix with $\left\{a_{1}, a_{2}, \ldots, a_{d}\right\}$ in the top row, 1s on the sub-diagonal, and 0s elsewhere. The characteristic polynomial for (5.32) is

$$
p(t)=\operatorname{det}(\lambda I-A)=\lambda^{d}-\Sigma_{k} a_{k} \lambda^{d-k}
$$

where $\mathrm{k}=1$ to $\mathrm{d}$ and the complex roots (not necessarily distinct) $\left\{\lambda_{\mathrm{i}}\right\}$ where $\mathrm{i}=1$ to $\mathrm{d}$ are the eigenvalues of $\mathrm{A}$.

If the variance is zero, the qualitative behavior of solutions to (5.32) is related to the magnitude of the eigenvalues. Suppose for simplicity that the eigenvalues of A are distinct. Then for the initial vector $\mathrm{X}(0)=\Sigma \mathrm{a}_{\mathrm{i}} \mathrm{v}_{\mathrm{i}}$ where $\mathrm{v}_{\mathrm{i}}$ is an eigenvector and the solution to (5.32) is $X(t)=A^{t} X(0)=\Sigma a_{i} i^{t} v_{i}$. Hence

$$
\|X(t)\|_{1 / t}=\left\|A^{t} X(0)\right\|_{1 / t} \rightarrow \max \left\{\left|\lambda_{i}\right|: a_{i} \neq 0\right\} \quad \text { as } t \rightarrow \infty
$$

The Lyapunov exponents for (5.32) are the numbers $\lambda_{i}=\log \left\{\left|\lambda_{i}\right|\right\}$, arranged so that the form an increasing sequence in absolute value such as $\left|\lambda_{1}\right|<\left|\lambda_{\mathrm{I}}\right|<\ldots<\left|\lambda_{\mathrm{d}}\right|$. In terms of (5.34), the Lyapunov exponents give the possible asymptotic rates of exponential increase (or decrease) for solutions (5.32) with $s=0$. The asymptotic growth rate with be $\lambda_{1}$ for almost all initial vectors, $\mathrm{X}(0)$. 
Let $J(t)=D F(X(t))$ the Jacobian matrix of $F$ at $X(t)$. The Lyapunov exponents for the nonlinear dynamical system, $\mathrm{X}(\mathrm{t})=\mathrm{F}(\mathrm{X}(\mathrm{t}-1))+\mathrm{e}(\mathrm{t}), \mathrm{X}(0)$ given and $\mathrm{X}(\mathrm{t})$ are defined as

$$
l(X(0), Y(0))=\lim _{t \rightarrow \infty}(1 / t) \log \|Q(t) Y(0)\|
$$

where $\mathrm{Q}(\mathrm{t}) \mathrm{Y}(0)$ are solutions to the linear system

$$
\mathrm{Y}(\mathrm{t})=\mathrm{J}(\mathrm{t}) \mathrm{Y}(\mathrm{t}-1)
$$

That is , $\mathrm{Q}(\mathrm{t})=\mathrm{J}(\mathrm{t}-1) \mathrm{J}(\mathrm{t}-2) \ldots \mathrm{J}(0)$ or $\mathrm{Q}(\mathrm{t})=\mathrm{DF}(\mathrm{X}(\mathrm{t}-1)) \mathrm{DF}(\mathrm{X}(\mathrm{t}-2)) \ldots \mathrm{DF}(\mathrm{X}(0))$.

Because the Lyapunov exponents for the nonlinear system are defined to be the Lyapunov exponents of the tangent map system (5.34), the significance of Lyapunov exponents for nonlinear systems is their relationship to sensitive dependence on initial conditions which is the defining characteristic of chaos. Let $\mathrm{X}_{1}(\mathrm{t})$ and $\mathrm{X}_{2}(\mathrm{t})$ be solutions to (5.32) that differ only in their initial values; that is,

$$
X_{i}(t)=F\left(X_{i}(t-1)\right)+e(t), x_{i}(0) \text { given for } i=1,2
$$

If the initial perturbation, $Y(0)=\left(X_{1}(0)-X_{2}(0)\right)$ is sufficiently small, then the subsequent separation $Y(t)=\left(X_{1}(t)-X_{2}(t)\right)$ can be approximated by linearization,

$$
\mathrm{Y}(\mathrm{t})=\mathrm{F}_{\mathrm{t}-1}\left(\mathrm{X}_{1}(\mathrm{t}-1)\right)-\mathrm{F}_{\mathrm{t}-1}\left(\mathrm{X}_{2}(\mathrm{t}-1)\right) » \mathrm{~J}(\mathrm{t}-1) \mathrm{Y}(\mathrm{t}-1)
$$

Thus, the time evolution of a small perturbation to the state of the nonlinear system (5.32) is approximately by the linear system (5.36). In the formal limit of infinitesimally small $\mathrm{Y}(0)$, the approximation becomes exact. Thus, the Lyapunov exponents of the nonlinear system disclose the growth rates of local perturbations to the system. If $\lambda_{1}>0$, then almost all initially small perturbations grow exponentially over time and $\lambda_{1}>0$ indicates "sensitive dependence on initial conditions" in system (5.32). 
There are two classes of methods in use for estimating $\lambda_{1}$ from experimental or observational data. "Direct" methods proposed by Guckenheimer (1982) and successfully implemented by Wolf, Swift, Swinney, and Vastano (1985), are based on the assumption that $\mathrm{Y}(\mathrm{t})$ in (5.11) will grow exponentially at rate $\lambda_{1}$. $\mathrm{Y}(0)$ as defined previously cannot be observed, however, because our time series data is only a single realization. Thus, the growth rate of an infinitesimal perturbation is estimated by tracking the evolution of the difference between initially close observed data points. The available time series data, $X(t)$ are searched for pairs of times $\left(t_{1}, t_{2}\right)$ where ||$X\left(t_{1}\right)-X\left(t_{2}\right)||$ is sufficiently small. Then the growth of $Y(t)=|| X\left(t_{1}+t\right)-$ $\mathrm{X}\left(\mathrm{t}_{2}+\mathrm{t}\right)||$ is recorded until the trajectories diverge beyond some preset limit or time. The data are then searched for a replacement point $X\left(t_{3}\right)$ that is sufficiently near $X\left(t_{1}+t\right)$ and also sufficiently near the line connecting $X\left(t_{1}+t\right)$ and $X\left(t_{2}+t\right)$. The process is repeated, and the average divergence rate over the entire data set is the estimate of $\lambda_{1}$.

This method constructs a lower limit $\mathrm{L}^{*}$ of the maximum Lyapunov exponent $\mathrm{L}^{\text {Emax }}$. The procedure, similar to the correlation integral, is to select all pairs of nearby points such that,

$$
\mathrm{z}_{0}=|| \mathrm{X}_{\mathrm{i}}-\mathrm{X}_{\mathrm{j}}||<\varepsilon
$$

and calculate the distances of these points after $t$ time steps,

$$
z_{t}=|| X_{i+t}-X_{j+t}||
$$

A stretching factor from any pair of adjacent points can be obtained by

$$
\mathrm{d}_{\mathrm{t}}=\mathrm{z}_{\mathrm{t}} / \mathrm{z}_{0}
$$

where averaging leads to an estimate, $\mathrm{L}^{*}$,

$$
L^{*}(t)=T^{-1} \Sigma_{i, j} \ln d_{t}
$$


Equation (5.41) gives the lower limit, $\mathrm{L}^{*}(\mathrm{t})$ that is dependent on the number of time steps t. For small $t, L^{*}(t)$ oscillates which suggests that $t$ should be larger than the attractor's dimension. Because of this, the lower limit will underestimate $\mathrm{L}^{\text {Emax }}$; however, a positive $\mathrm{L}^{*}$ will imply a positive Lyapunov exponent.

The method employed by Wolf et al. (1985) consists of examining the first m-dimensional vector, $\mathrm{X}_{1}$. The time path traced by this vector is labeled as the reference path. Similar to the above method, the m-dimensional vector space is searched for the nearest vector, $\mathrm{X}_{\mathrm{j}}$. A time delay, dt, is chosen such that we can measure the distance between $\mathrm{X}_{1+\mathrm{dt}}$ and $\mathrm{X}_{\mathrm{j}+\mathrm{dt}}$. The exponential rate of divergence per time period is to compute from the ratio of the original distance between the two vectors and after the time delay. This information is stored and the new reference point becomes $X_{1+d t}$ and then we look for the next vector $X_{k}$. This vector $X_{k}$ is selected by finding a vector that is closer to $X_{j+d t}$ than $X_{1+d t}$ was. Once $X_{k}$ is selected then the same procedure is repeated, that is, the exponential divergence is calculated from the ratio and a new reference is selected. The procedure is completed when all the data available for the reference path are exhausted. These stored rates are then averaged which generates an estimate of the largest Lyapunov exponent, $\mathrm{L}^{\mathrm{emax}}$

Four parameters are to be chosen for this method:

- an embedding dimension and time lag for the reconstruction;

- evolution time, i.e. the interval over which one follows two pieces of the trajectory;

- minimum acceptable separation between points that are to be followed;

- maximum acceptable separation.

In "Jacobian" methods the data are used to estimate the Jacobians $\operatorname{DF}(\mathrm{X}(\mathrm{t}))$ and $\lambda_{1}$ is calculated from the estimated Jacobians. The Jacobian methods proposed by Eckmann and 
Ruelle (1985), as an implementation based on the linear regression were tested. Basically, in order to estimate $\operatorname{DF}(X(t))$, identification is needed of all data points $\mathrm{X}(\mathrm{s})$ lying within some distance of $\mathrm{X}(\mathrm{t})$. The approximation

$$
X(s+1)-X(t+1)=F(X(s))-F(X(t)) \approx D F(X(t))(X(s)-X(t))
$$

of (5.42) is based on the difference of the linear model $X(s+1)-X(t+1) \approx A(t)(X(s)-X(t))$. Assuming that sufficiently many $\mathrm{X}(\mathrm{s})$ exist, then $\mathrm{A}(\mathrm{t})$ can be determined from a least squares fit to the differences. This least squares solution provides an estimate for $\operatorname{DF}(\mathrm{X}(\mathrm{t}))$ which is then substituted into (5.42).

In tests with simulated data from low-dimensional systems, the direct and linear Jacobian methods both appear adequate if there is an abundant supply of very accurate data, the system is deterministic, or nearly so, and the correct dimension is known (Mayer-Kress 1986). Because the correct dimension is unknown, a common strategy is to increase the dimension until estimates of $\lambda_{1}$ reach a stable plateau. The linear Jacobian method can generate spurious exponents when the dimension is too large, so this strategy may fail. Eckmann et al. (1986) claimed that the spurious exponents can be eliminated by proper choice of the method's free parameters, but it appears that discrimination between "real" and "spurious" exponents is essentially ad hoc and requires much trial and error in tuning the free parameters.

The direct methods are less suited to mixed stochastic/nonlinear dynamics. It is easy to see that the direct method is positively biased when $\mathrm{s}>0$. To eliminate bias, the divergence must be fitted to accommodate a more complicated growth model, which allows for stochastic dynamics. If a model such as (5.32) is believed to represent the dynamics, then the appropriate model for the divergence would involve $\mathrm{X}(\mathrm{t})$ and $\mathrm{Y}(\mathrm{t})$ dependence in the growth of $\mathrm{Y}(\mathrm{t})$. 
Consequently, the Jacobian methods are preferable for stochastic nonlinear systems, but either must incorporate a criterion for choosing the dimension or else use an estimator for $\mathrm{DF}(\mathrm{X}(\mathrm{t}))$ that is less sensitive to extraneous lags in the model. Procedures for fitting nonlinear time series models to chaotic data have been very successful in generating estimators for $\mathrm{F}$.

\section{Conclusions}

Overall, one can avoid estimating all these measures by exploiting the relationships that exist among measures. These relationships exist in the form of inequalities that are illustrated in Table 4. Since these dimension measures give some indication of the number of variables needed to describe the system that gave the time path of the variable in question, the table provides upper and lower bounds, $C_{D}$ and $D_{E}$, for this number. That is the estimation of one or both of these measures can give the researcher this information. Although there exist many modifications to these tests and to this point questions concerning data limitations and questions of power have only begun to surface, the following list gives the empirical economist a methodology that can classify an economic time series as stochastic, periodic or aperiodic (chaotic).

The estimation of Lyapunov exponents and related measures has been historically plagued by the lack of a distribution theory. In addition the small and noisy data sets that are common in economics decrease the credibility in the estimated values and dissolve restructuring possibilities. Furthermore, the existence of near-unit root stochastic processes common in economic and financial data series can yield seemingly low dimension estimates and seemingly positive lyapunov exponent estimates. 


\section{CHAPTER 6: EMPIRICAL RESULTS}

Sometimes truth comes riding into history on the back of error. -Reinhold Niebuhr

\section{Introduction}

The purpose of this chapter is to determine whether or not there is strong statistical evidence for a generalization regarding the presence of chaos in agricultural commodity prices. The previous chapters have provided the background and framework for the analysis.

The agricultural commodity price behavior of interest here relates to the commodities exchanged on the major international markets for bananas, beef, coffee, soybeans, wool and wheat. Each commodity is representative of a specific commodity group. All groups, except the metals group are represented in the analysis (Table 6.1). Definitions of the prices quoted on the above markets are listed in Appendix Table A.1. 
Table 6.1 Classification of Commodity Prices

Agricultural Raw Materials

Cotton

WOOL

Sisal

Jute

Rubber

Hides and Skins

Tropical Timber

Food

WHEAT

Maize

Rice

Sugar

BEEF

BANANAS

Pepper

Soybean Meal

Fish Meal

Tropical Beverages

\section{COFFEE}

Cocoa

Tea
Minerals, Ores, and Metals

Phosphate Rock

Manganese Ore

Iron Ore

Aluminum

Copper

Lead

Tin

Tungsten

Zinc

Vegetable Oilseeds and Oils

SOYBEANS

Soybean Oil

Sunflower Oil

Ground nuts

Palm Kernels

Palm Kernel Oil

Palm Oil

Linseed Oil

The sampling interval chosen for this analysis is monthly due to the need for sufficient observations for the power properties of the tests previously described, the results of which are included in this chapter. The data are from the UNCTAD Monthly Commodity Price Bulletin, published by the United Nations in Geneva. These empirical prices series are sampled monthly, i.e., they reflect the average of daily prices over the period January 1960 to June 1994. 


\section{Graphical Analysis}

In order to provide an intuitive framework with which to analyze these agricultural commodity prices, a visual portrait for each price series is presented (Figures 6.1-6.6) over the period 1960.01 to 1994.06 . Figure 1 demonstrates that over this chosen period banana price levels exhibit a time-dependent varying amplitude. Clearly, the price series is nonstationary with an increasing mean and variance. Upon closer inspection, it can be hypothesized that the variance is functionally related to the mean of the price series.

Figure 6.1 Monthly price levels for bananas

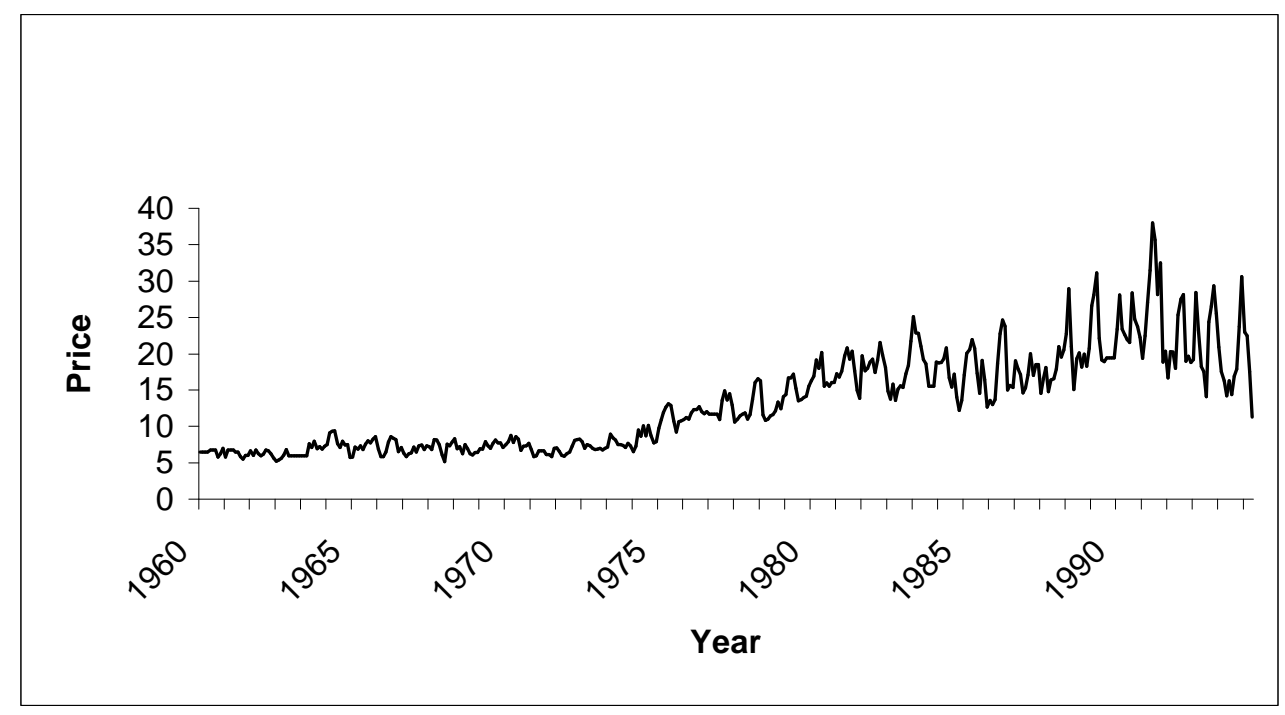


Figure 6.2 Monthly price levels for beef

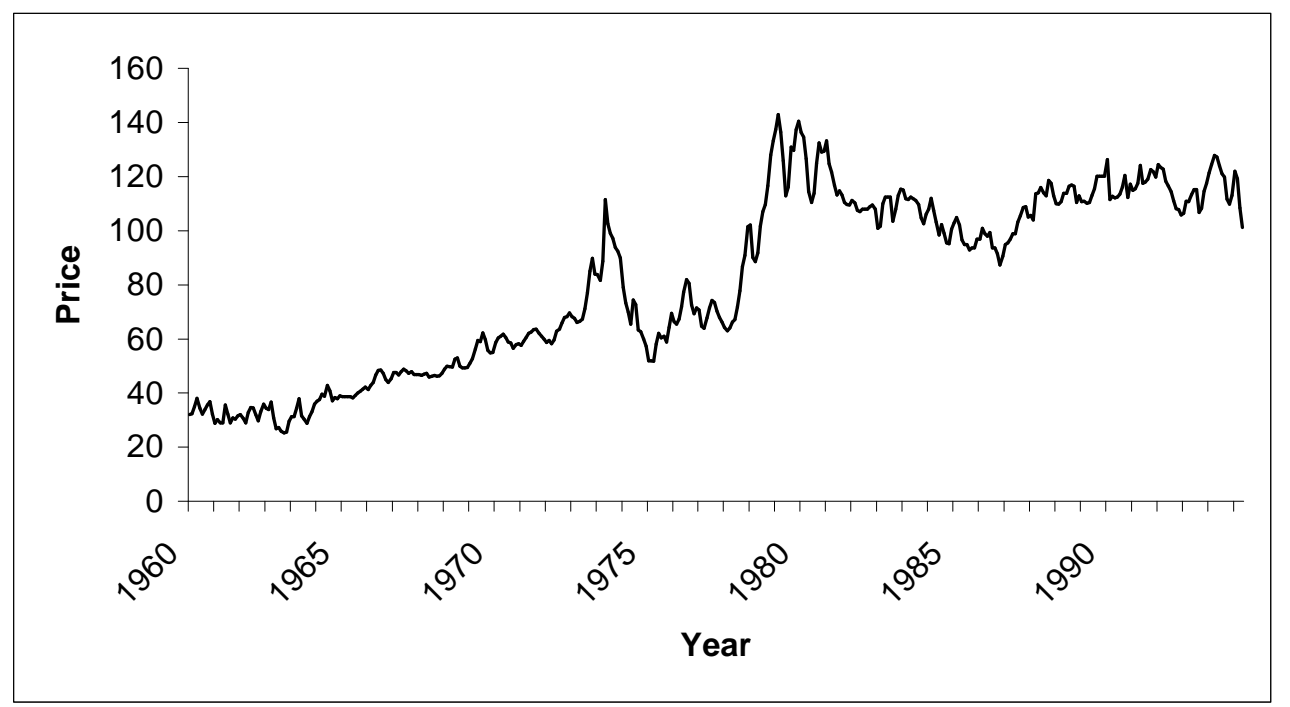

Figure 6.3 Monthly price levels for coffee

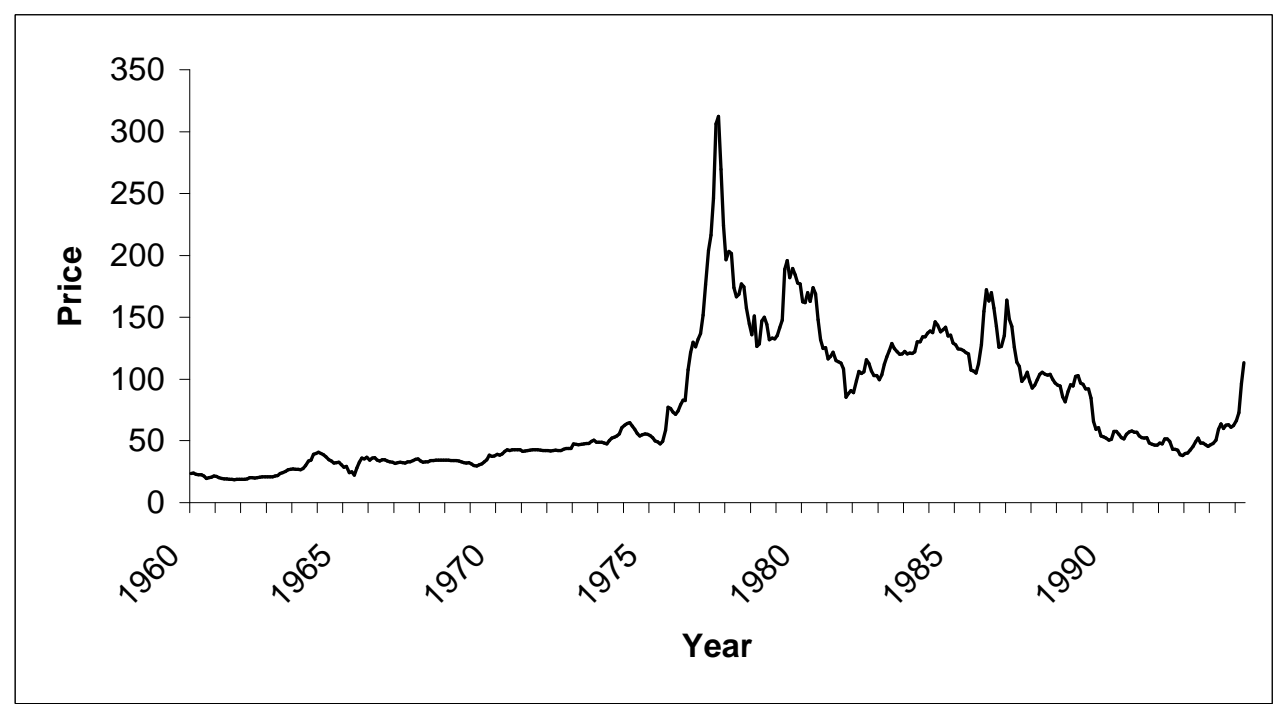


Figure 6.4 Monthly price levels for soybeans

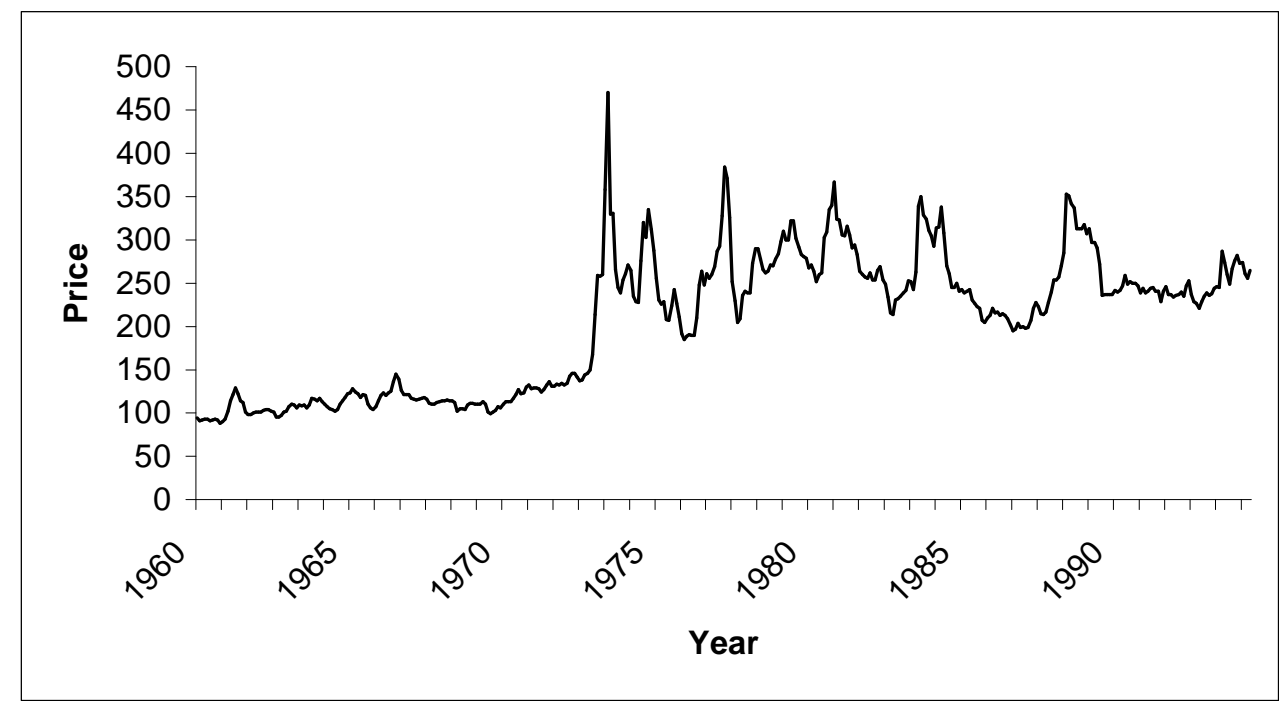

Figure 6.5 Monthly price levels for wool

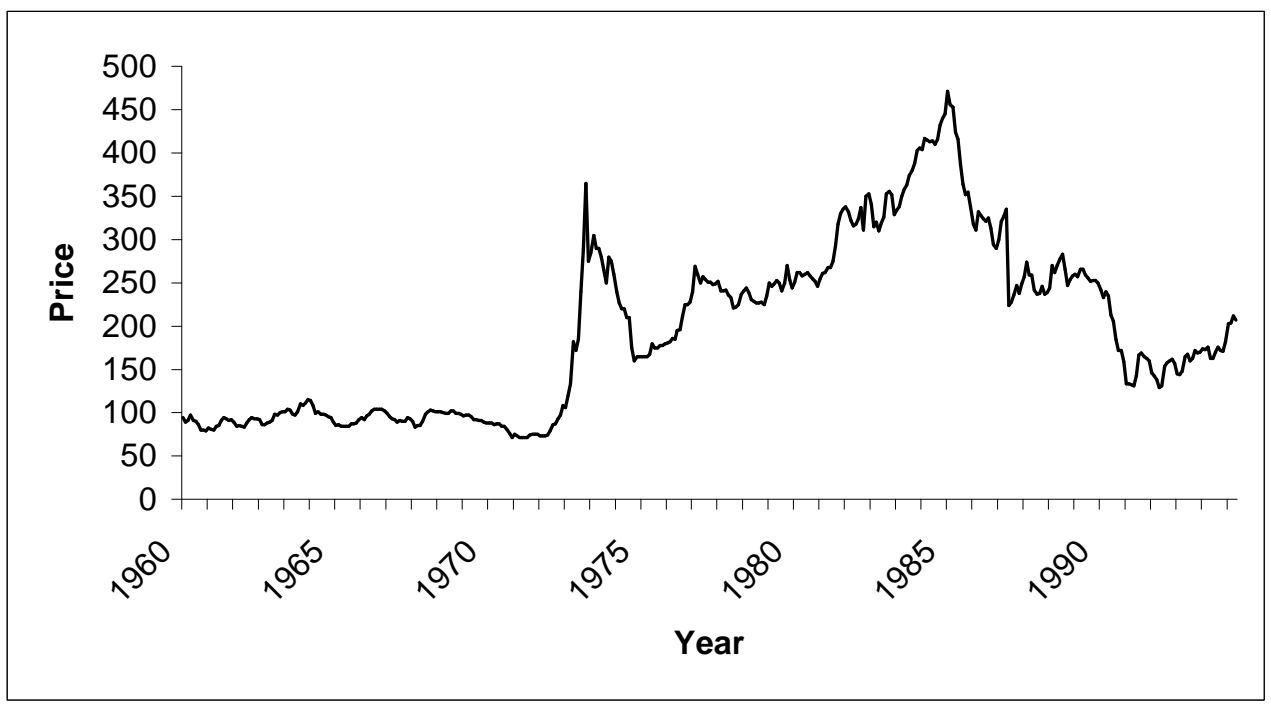


Figure 6.6 Monthly price levels for wheat

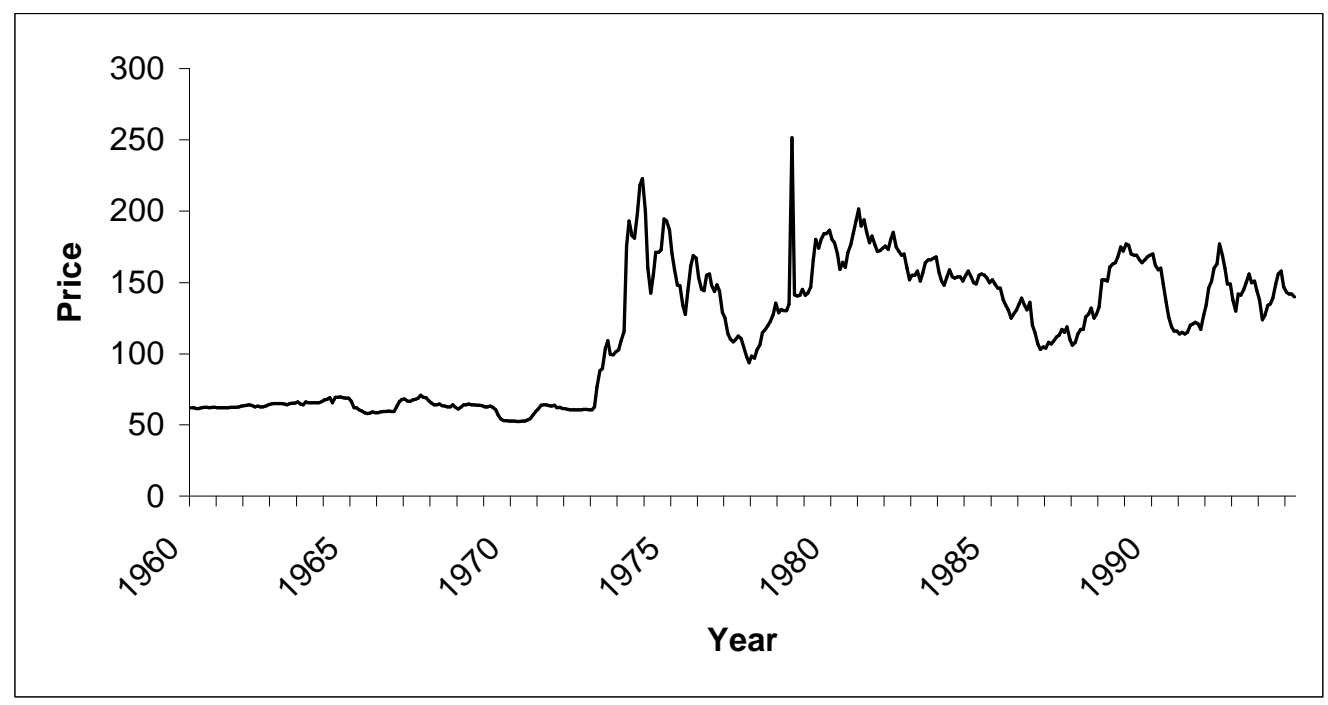

Figure 6.2 illustrates the path of beef prices over the chosen period. The first noticeable feature is the two peaks during time period in the seventies, a time of high inflation, triggered by oil price shocks. An especially turbulent time for beef prices, a trend is coupled with the instability in the variance. Figure 6.3 , for coffee prices demonstrates a similar feature to that of beef inasmuch as the stochastic shocks present in the seventies are also here. One feature that is dissimilar is the absence of any apparent trend component to the series. Although the variance appears to be non-constant, the relative magnitude of the series took a considerable amount of time to rebound from the previous year. Market intervention measures may be responsible for the damping effect illustrated in the late 1980s.

Figure 6.4 portrays the price level movements of soybeans. Again a similar pattern is emerges with respect to the first inflationary period, revealing that rising petroleum prices may 
have had a substantial impact upon the levels of this and other price series. For soybeans, it is obvious that while an increasing mean is not present, a transition has occurred from one level to another. The relative stability, e.g. variance, of the price has increased but may not be a function of the mean. In fact, the frequency of the price changes has seemed to increase in latter periods.

Figure 6.5 for wool prices is very similar to coffee and beef; however, the figure does illustrate a unique price movement that clearly demonstrates a non-constant variance. In fact, this variance does not appear to be related to the mean of the series.

Figure 6.6 again illustrates this similar pattern. While most of the price series illustrate a changing mean, it appears that the mean is more related to possibly relate to a stochastic shock, then to have emerged from the price movements themselves. Clearly, as is the case here, once the stochastic shock has been absorbed by the system, it may have changed if not the frequency of price movement, certainly the relative magnitude of the series with respect to the variance. Rather than state that prices returned to some equilibrium value, the graphs indicate that the initial impetus into unfamiliar stochasticity has generated a different environment.

Because of the properties inherent in the visual inspection of these prices series, a transformation is necessary to render the moments of these empirical distributions stationary. ${ }^{53}$ Because all series possessed a time dependent variance component which maybe a function of the mean, a natural $\log$ transformation was performed on each of the series. Since a trend in mean is anticipated and visually verified in many of the price series, the log series were differenced to reflect growth rates, $r(t)$. The transformed data were then seasonally adjusted using the additive technique of the Census X-11 procedure. This transformed data demonstrated 
in Figures 6.7 to 6.12 portrays the magnitude of the growth rates over the uniform range of 40 percent. For Figures 6.11 and 6.12, wool and wheat prices exhibit outliers, however, these are negligible. ${ }^{54}$

Figure 6.7 Monthly Seasonally Adjusted Growth Rates for Bananas

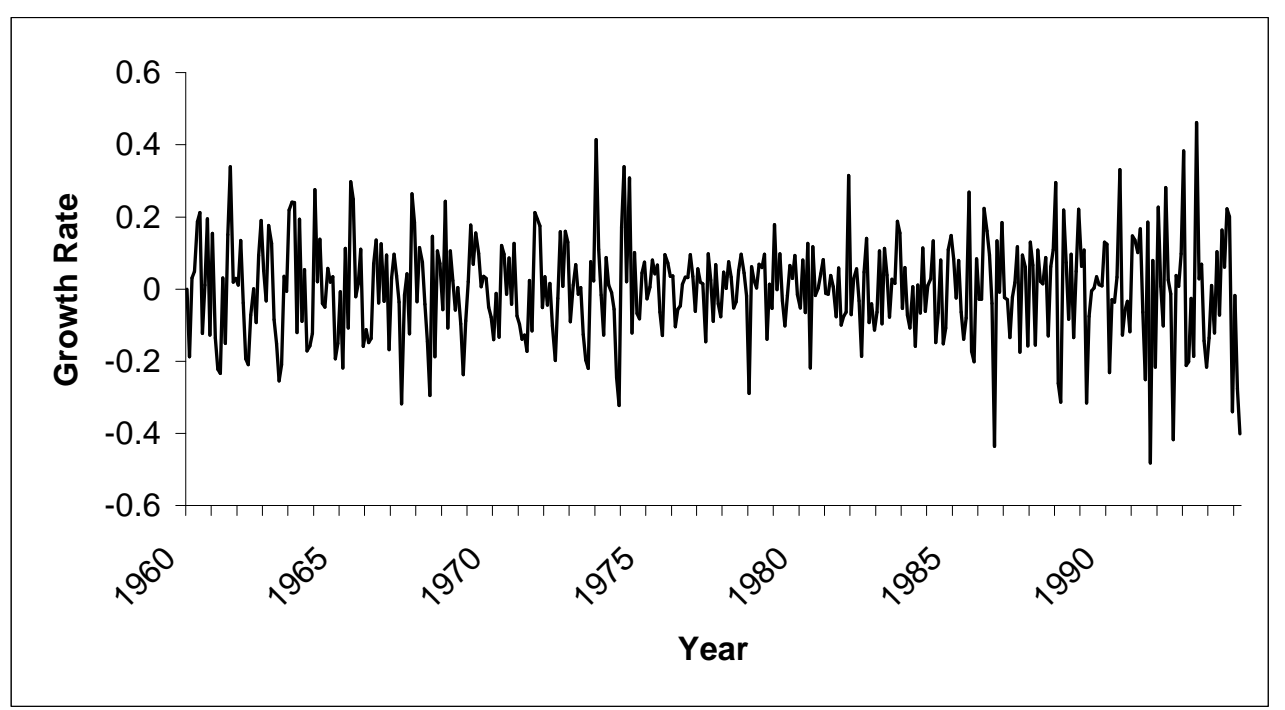

${ }^{53}$ Typical stationairty tests of the Dickey Fuller (1979) variety were performed on all series. The acceptance of the null hypothesis of a unit root was accepted for each series necessitating the employment of transformations.

${ }^{54}$ The analysis contained in these sections was robust to both the inclusion and exclusion of outliers. In the case of exclusion, the analysis was repeated with only those observations that did not exceed $4 \sigma$ from other observations contained in the data set. 
Figure 6.8 Monthly Seasonally Adjusted Growth Rates for Beef

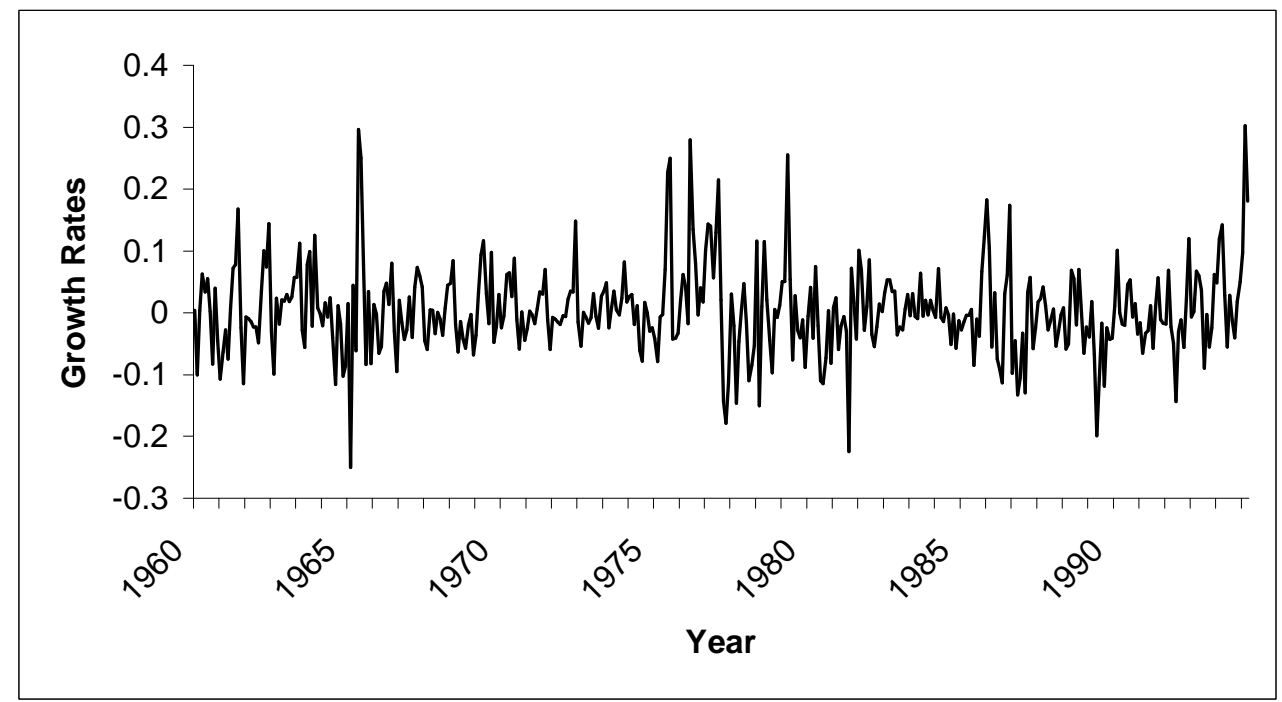

Figure 6.9 Monthly Seasonally Adjusted Growth Rates for Coffee

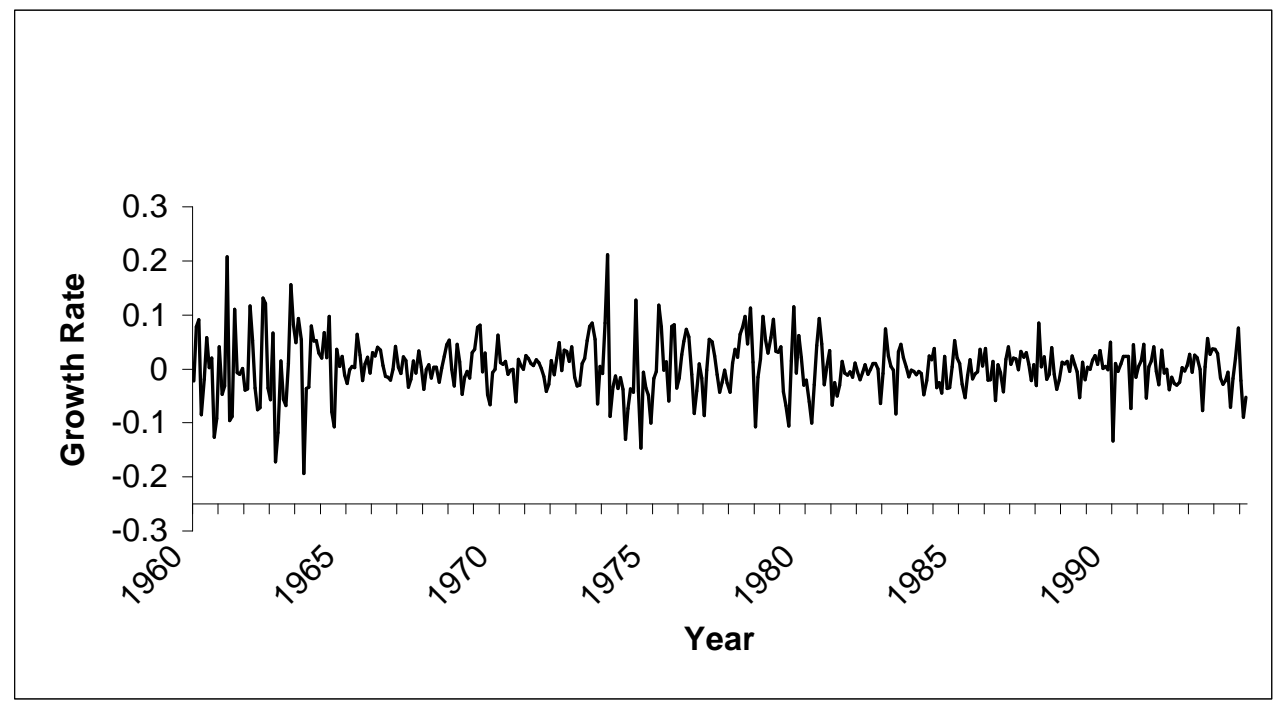


Figure 6.10 Monthly Seasonally Adjusted Growth Rates for Soybeans

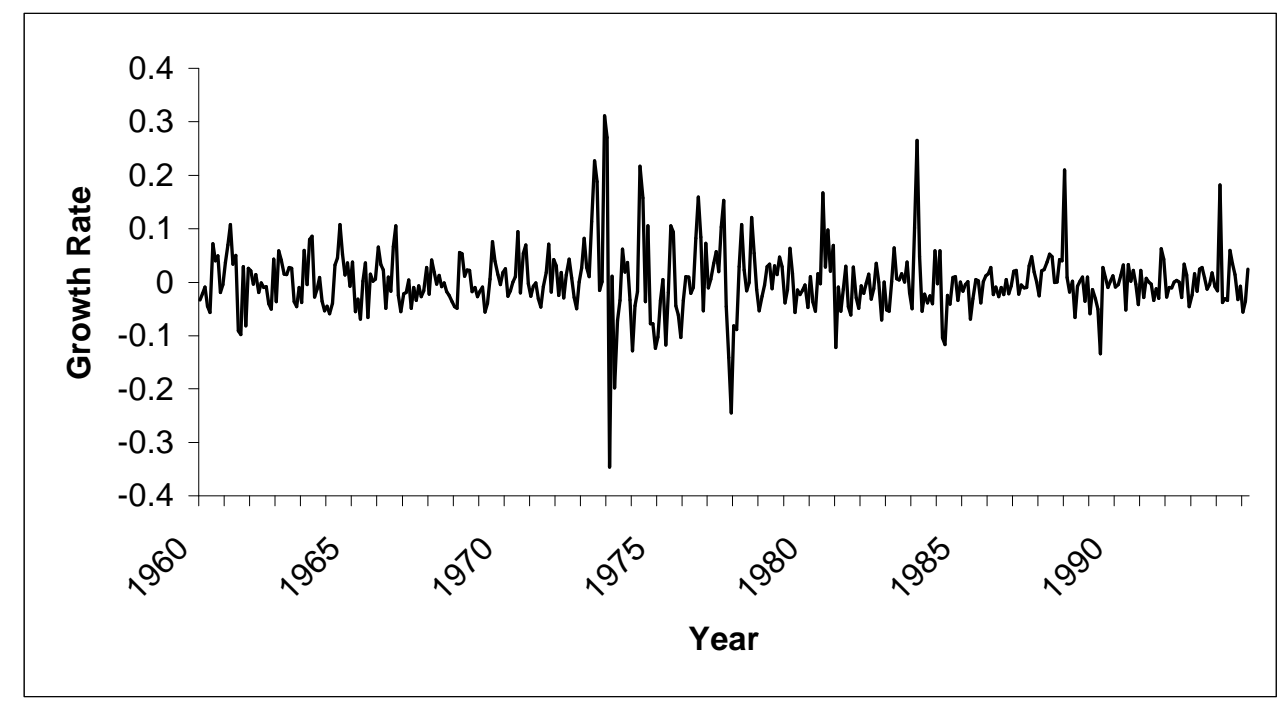

Figure 6.11 Monthly Seasonally Adjusted Growth Rates for Wool

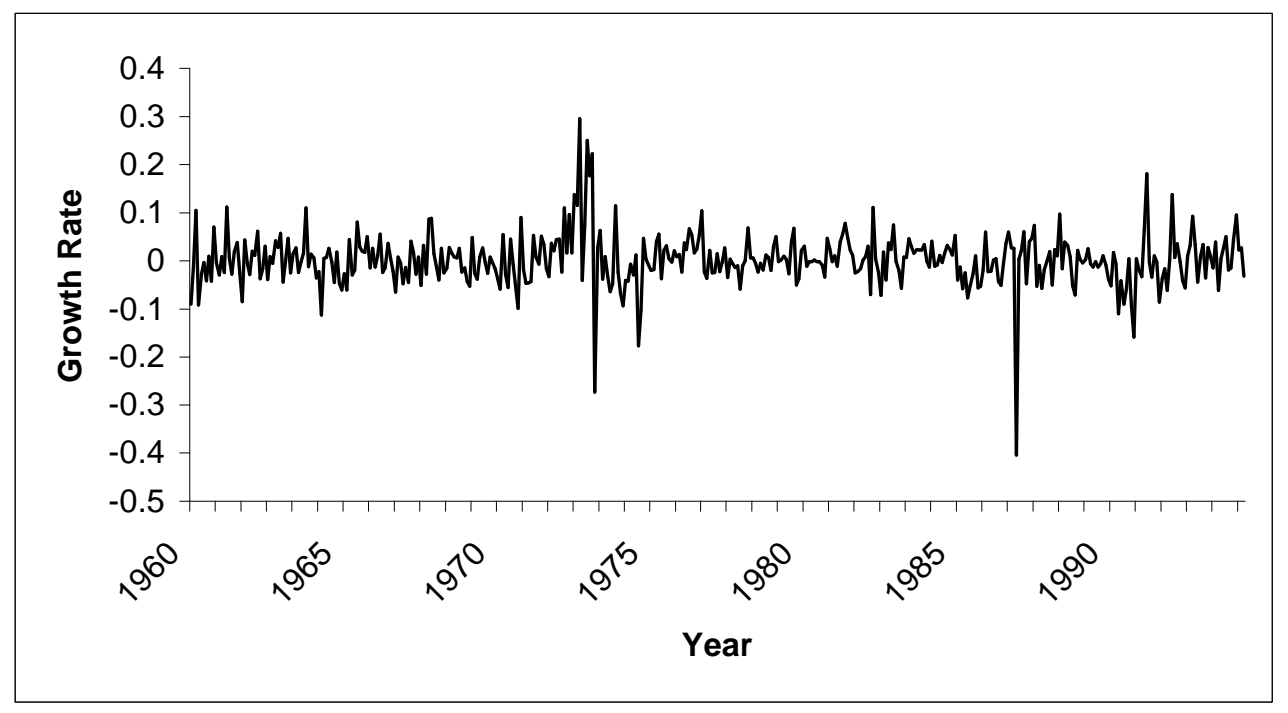


Figure 6.12 Monthly Seasonally Adjusted Growth Rates for Wheat

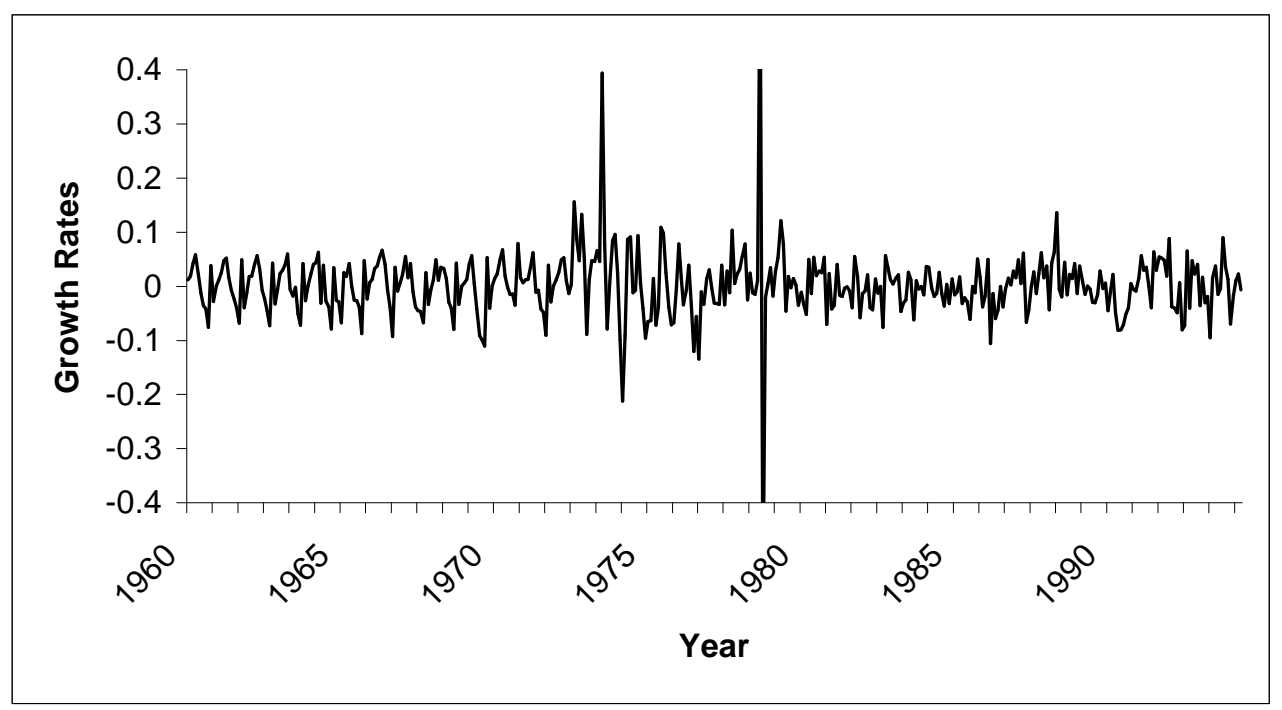

\section{Statistical Analysis}

Because of the difficulties present in visual inspection, attention is given to statistical measures. Table 6.2 disclosures the summary statistics of seasonally adjusted growth rates. Clearly, all growth rates are non-normal, as is to be expected. 
Table 6.2 Summary Statistics of Seasonally Adjusted Growth Rates (1960.02-1994.06)

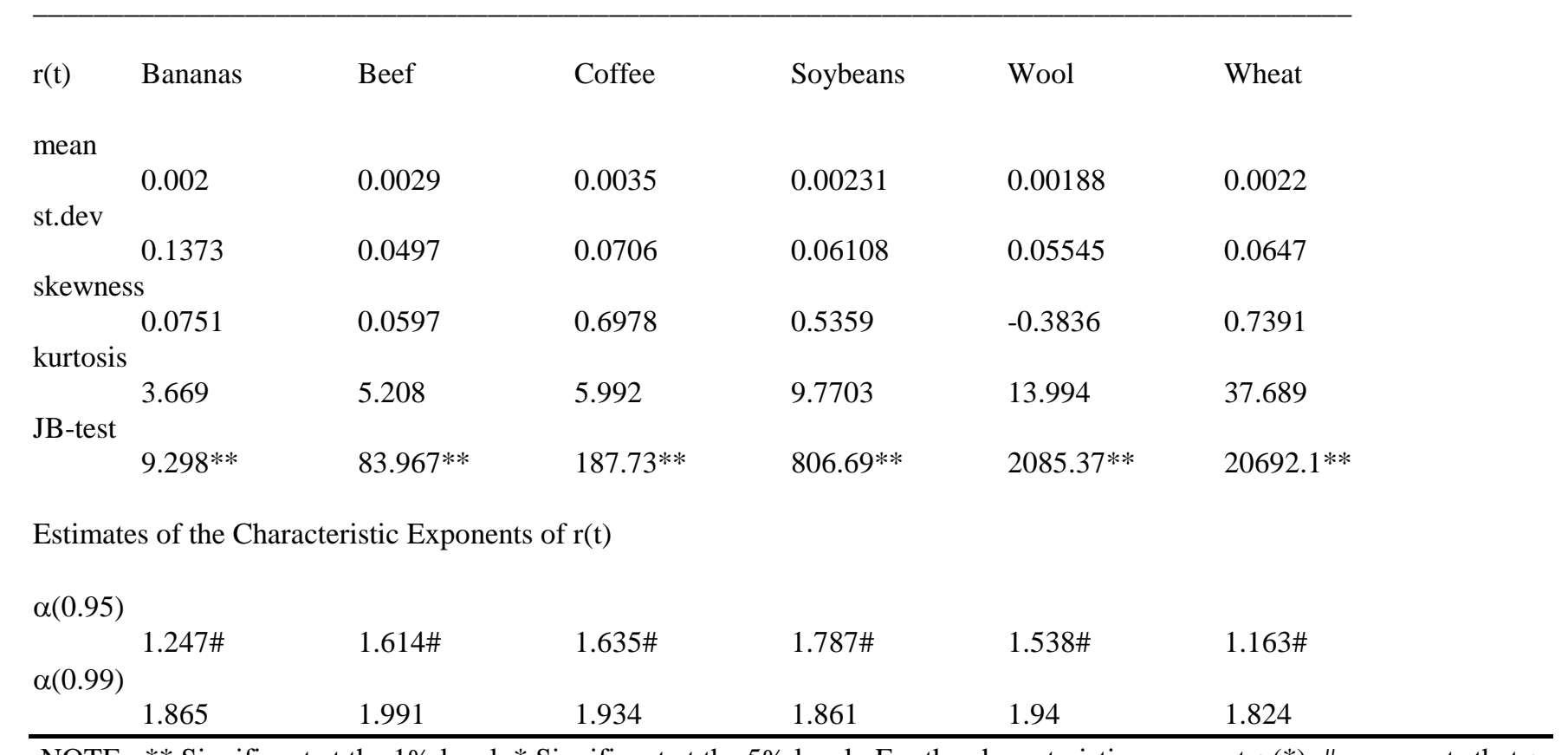

NOTE: $* *$ Significant at the $1 \%$ level, $*$ Significant at the $5 \%$ level. For the characteristic exponent $\alpha(*)$, \# represents that $\alpha$ is significantly less than 2 at the 0.05 level. 
Each figure calculates the gaussian expectation for interval of growth rates through 4 standard deviations. The graph represents the the normal curve with the scaled growth rates.

Figure 6.13 Histogram of Seasonally Adjusted Growth Rates for Bananas

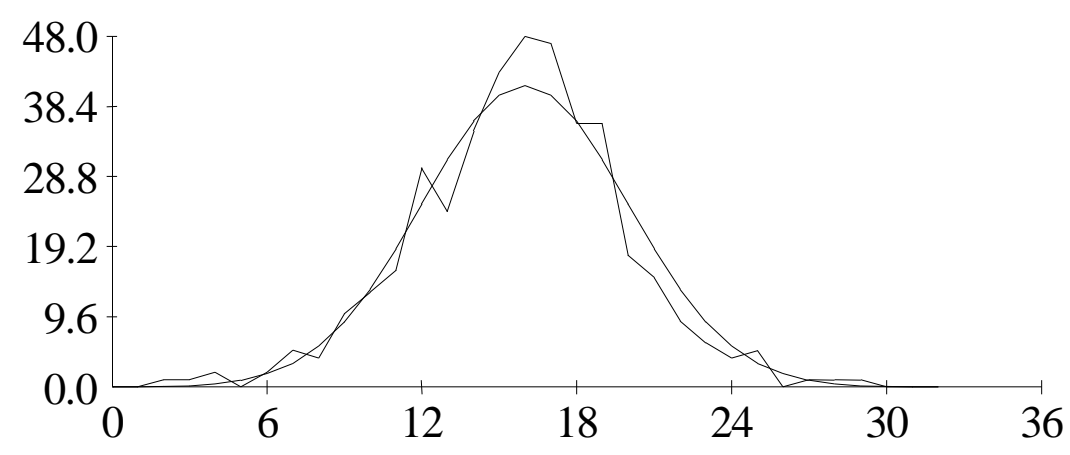

Figure 6.14 Histogram of Seasonally Adjusted Growth Rates for Beef

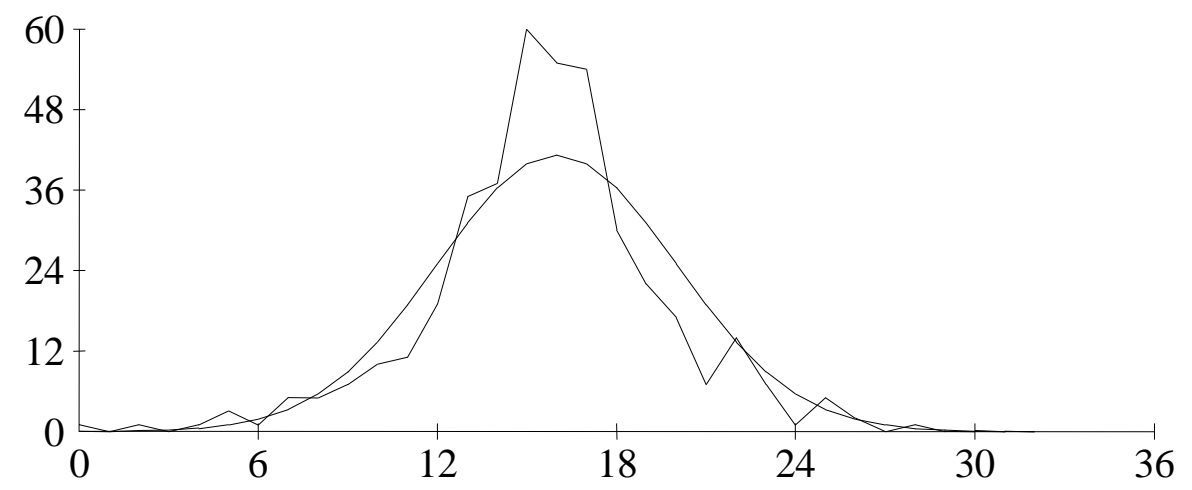


Figure 6.15 Histogram of Seasonally Adjusted Growth Rates for Coffee

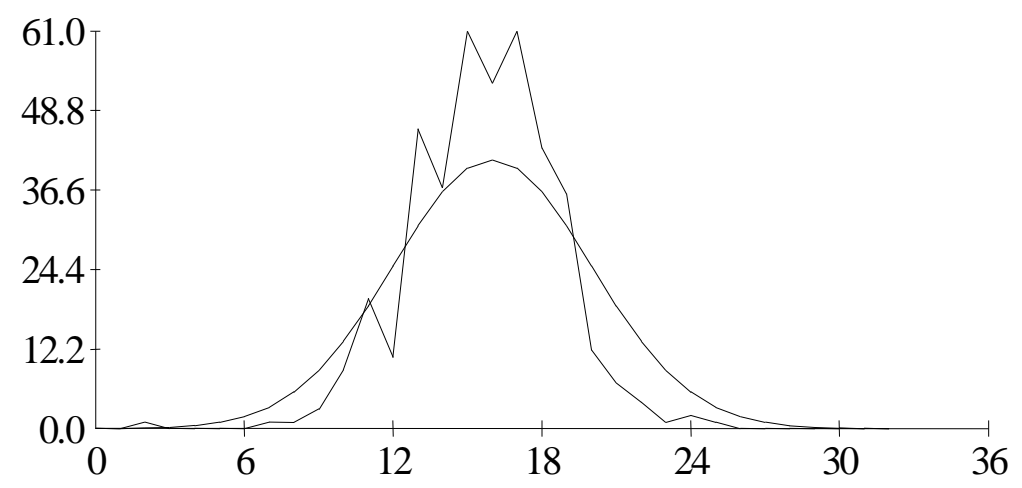

Figure 6.16 Histogram of Seasonally Adjusted Growth Rates for Wheat

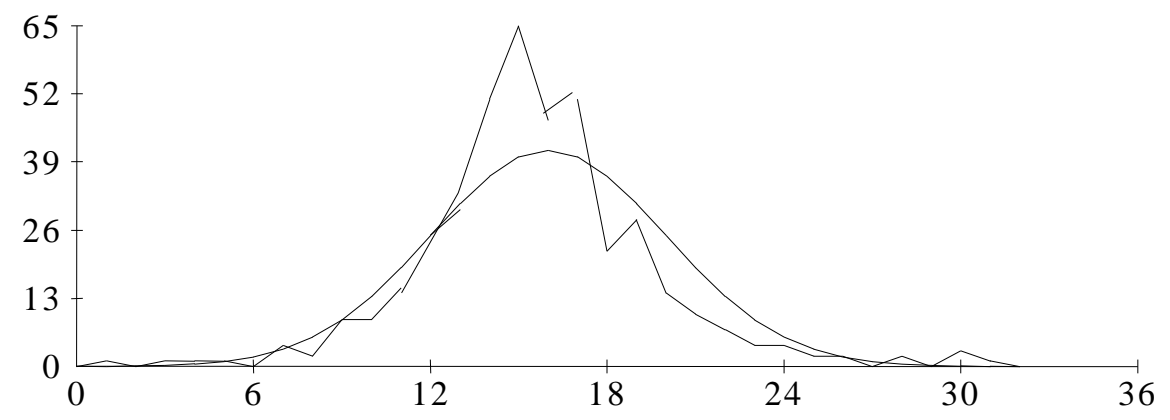


Figure 6.13 shows the histogram for seasonally adjusted bananas growth rates. Notice the presence of a couple of modes to the lift and right of the center of the distribution. This suggests that there may be a bimodal distribution for these growth rates.

Figure 6.14 suggests that beef growth rates may have a similar bimodal distribution at the tails of the distribution. Figure 6.15 shows the histogram results for seasonally adjusted growth rates for coffee prices. Notice the presence of a multi-modal distribution for these prices. It should be expected that some form of dependence similar to that of beef prices is present.

Clearly, wheat prices demonstrate a bimodal distribution. In Figure 6.16, notice the existence of multiple modes that suggest the potential of chaotic behavior. The existence of these modes is illustrative of many different trajectories that occur frequently enough to warrant different modes.

Based on these visual portraits, it is apparent that the chosen prices are nonstationary, leptokurtic and may indeed have chaotic properties, especially in the case of wheat. To provide stronger evidence for this conjecture, Table 6.2 summarizes the results of applying the DickeyFuller and Augmented Dickey-Fuller tests for unit roots to the original and to the transformed series. Lag values of 1 and 4 were chosen for the augmented test in order that a comparison could be made between the Dickey-Fuller critical values and those of McKinnon (1990). As the results show, these prices are integrated of order one, I(1), and quite robust to a variety of 
different null hypotheses. In fact it comes as no surprise that this is the case for commodity prices in general. ${ }^{55}$

The results demonstrate that the growth rates for wheat are less variable than other growth rates. It is quite apparent from the results in this table that we have leptokurtic empirical distributions for these growth rates, which result in non-normality. At this point, an assumption is made that all growth rates are second order stationary and non-normal.

In order to determine the existence of cycles in the seasonally adjusted growth rates, a spectral analysis using a Parzen window was performed for each of the growth rates. The results for each are presented below.

${ }^{55}$ Clearly, it is expected that the unconditional moments of the price series are not stationary; however, it is a realistic assumption to make that the conditional moments will be. 
Table 6.3 Tests for Stationarity on Seasonally Adjusted Prices

\begin{tabular}{|c|c|c|c|c|c|c|}
\hline \multicolumn{7}{|l|}{ LEVELS } \\
\hline & BANANAS & BEEF & COFFEE & SOYBEAN & WOOL & WHEAT \\
\hline $\mathrm{C}$ & $-3.59 * *$ & -1.58 & -1.63 & -2.36 & -1.50 & -2.36 \\
\hline $\mathrm{T}$ & $-7.33^{* *}$ & -2.39 & -1.73 & -3.17 & -1.37 & -3.17 \\
\hline $\mathrm{N}$ & -1.44 & 0.25 & -0.45 & -0.39 & -0.27 & -0.47 \\
\hline \multicolumn{7}{|l|}{$\mathrm{ADF}$} \\
\hline $\mathrm{C}$ & $-3.28 *$ & -1.82 & -2.44 & -2.72 & -1.59 & -2.07 \\
\hline $\mathrm{T}$ & $-6.97 * *$ & -3.19 & -2.72 & $-3.80^{*}$ & -1.53 & -2.72 \\
\hline $\mathrm{N}$ & -1.27 & -0.01 & -1.02 & -0.57 & -0.35 & -0.29 \\
\hline \multicolumn{7}{|l|}{$\mathrm{k}=6$} \\
\hline $\mathrm{C}$ & -1.63 & -1.41 & -1.94 & -2.21 & -1.80 & -1.87 \\
\hline $\mathrm{T}$ & $-3.94 *$ & -2.25 & -2.09 & -3.08 & -1.87 & -2.52 \\
\hline $\mathrm{N}$ & -0.142 & 0.39 & -0.68 & -0.21 & -0.46 & -0.21 \\
\hline
\end{tabular}

Growth Rates

\begin{tabular}{crrrrrr} 
& BANANAS & BEEF & COFFEE & SOYBEAN & WOOL & WHEAT \\
$\mathrm{C}$ & $-19.66^{* *}$ & 16.59 & -13.69 & -15.61 & -17.85 & -20.61 \\
$\mathrm{~T}$ & $-19.65^{* *}$ & 16.60 & -13.96 & -15.61 & -17.88 & -20.59 \\
$\mathrm{~N}$ & $-19.68^{* *}$ & 16.57 & -13.94 & -15.63 & -17.89 & -20.61 \\
$\mathrm{ADF}$ & & & & & & \\
$\mathrm{k}=1$ & & & & & & \\
$\mathrm{C}$ & $-13.67^{* *}$ & 15.16 & -11.29 & 13.10 & -13.15 & -14.01 \\
$\mathrm{~T}$ & $-13.67^{* *}$ & 15.17 & -11.28 & -13.09 & -13.17 & -14.00 \\
$\mathrm{~N}$ & $-13.69^{* *}$ & 15.12 & -11.26 & -13.09 & -13.19 & -14.00 \\
$\mathrm{k}=6$ & & & & & & \\
$\mathrm{C}$ & $-12.65^{* *}$ & -9.23 & -8.14 & -8.75 & -6.66 & -8.83 \\
$\mathrm{~T}$ & -12.65 & -9.27 & -8.15 & -8.15 & -6.69 & -8.82 \\
$\mathrm{~N}$ & $-12.64^{*}$ & -9.13 & -8.09 & -8.09 & -6.14 & -8.80 \\
& & & & & & \\
\hline
\end{tabular}

Note: Signficant at the 0.01 level. *Significant at the 0.05 level. C=constant and no trend; $\mathrm{T}=$ trend with no constant; $\mathrm{N}=$ No contant and no trend. 
Figure 6.17 Power Spectrum of Seasonally Adjusted Growth Rates for Bananas

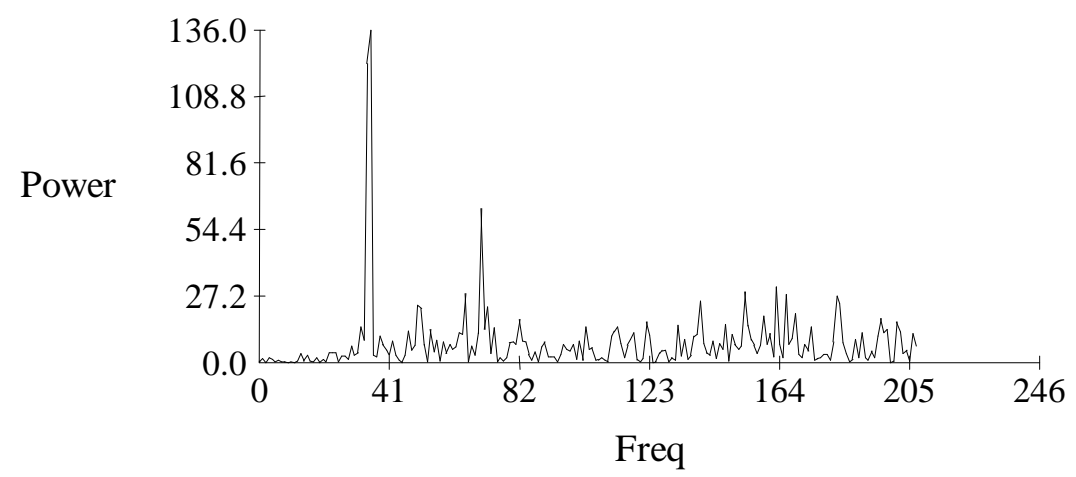

Figure 6.18 Power Spectrum of Seasonally Adjusted Growth Rates for Beef

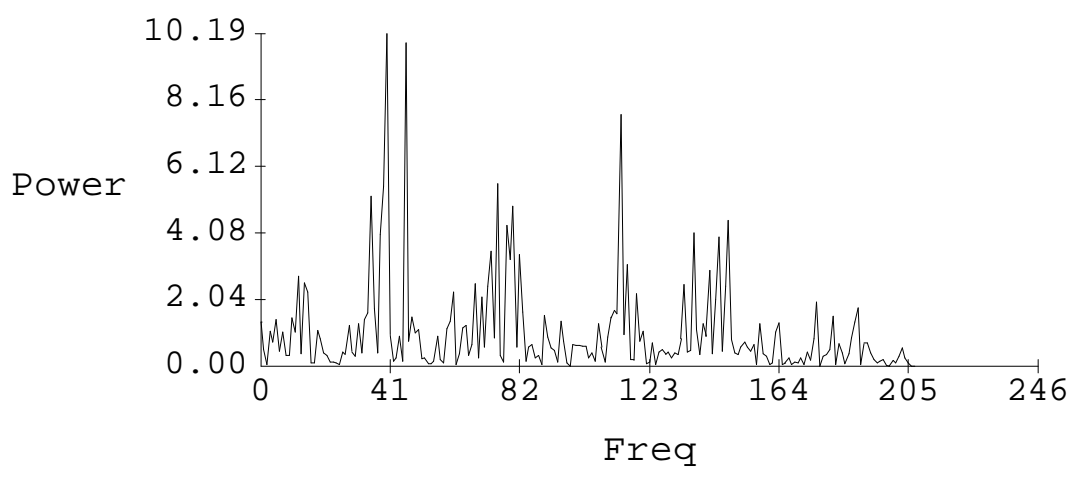

Figure 6.19 Power Spectrum of Seasonally Adjusted Growth Rates for Coffee

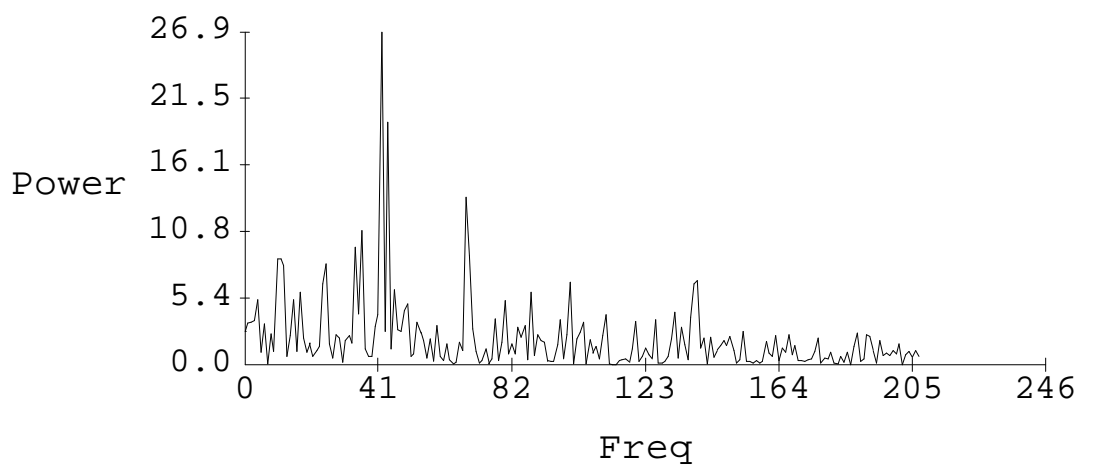


Figure 6.20 Power Spectrum of Seasonally Adjusted Growth Rates for Soybeans

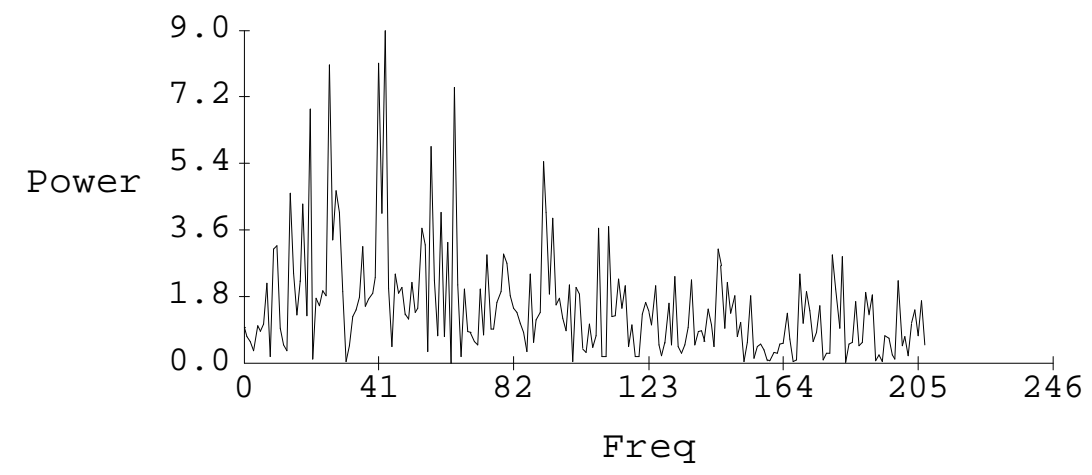

Figure 6.21 Power Spectrum of Seasonally Adjusted Growth Rates for Wool

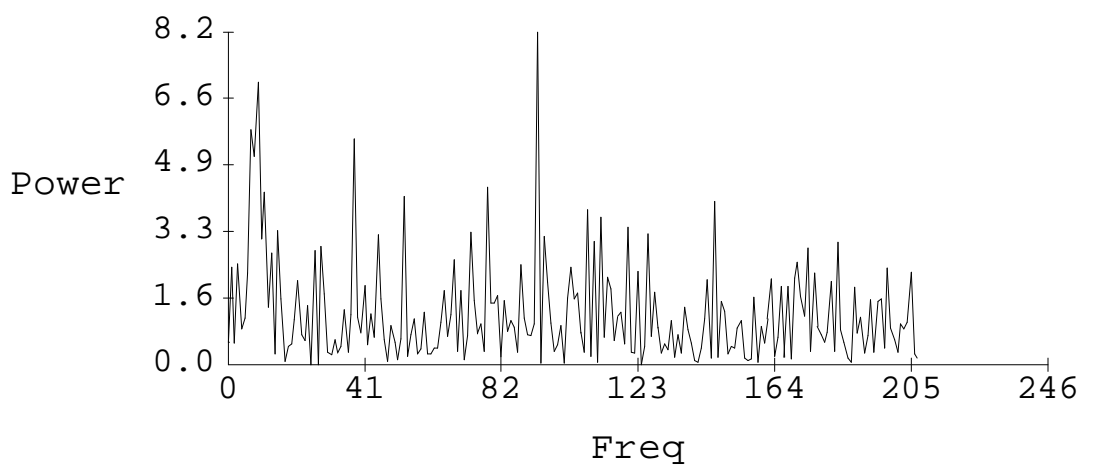

Figure 6.22 Power Spectrum of Seasonally Adjusted Growth Rates for Wheat

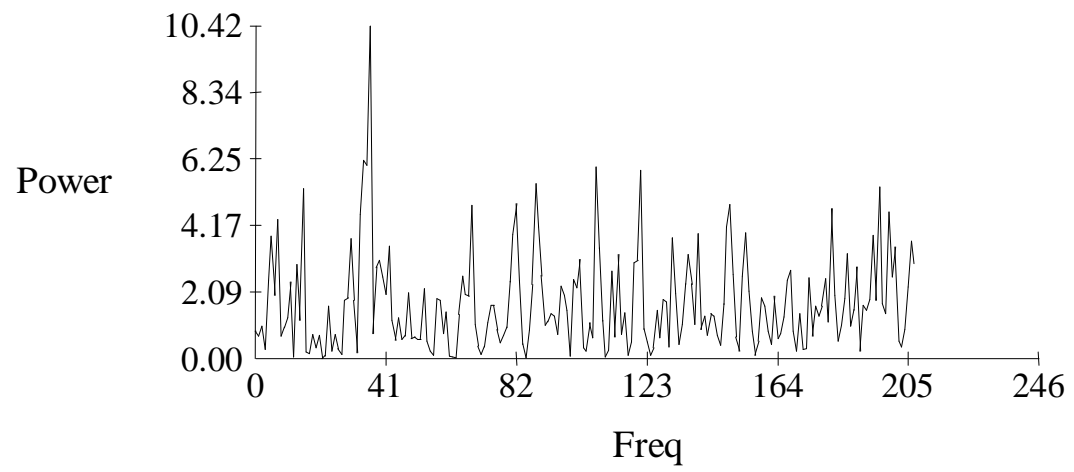


Figure 6.17 demonstrates the existence of high frequency cycles in bananas; however, as is seen, much of the series is obscured by noise. Figure 6.18 shows the spectrum for seasonally adjusted beef growth rates that display several peaks at varying different frequencies. The presence of both low and high frequencies presents problems in the modeling process.

Coffee prices, as Figure 6.19 demonstrates, are similar to these for beef where a substantial 3 and 1/2 year cycle is present. Figure 6.20 shows that soybean prices demonstrate a similar pattern to those for beef and coffee except that the cycles are more frequent and of a greater relative amplitude. Figure 6.21 for wool is different than the previous spectrums presented except that there is a substantially low frequency component in these seasonally adjusted growth rates. In addition, the presence of noise is substantial.

Figure 6.22 displays the spectrum for wheat prices. There appears to be a substantial 3 $1 / 2$ year cycle, and at other higher frequencies as well. Also, notice that at the highest frequency the power is increasing.

To obtain a more intitutive understanding about the frequency properties of these prices, a frequency decomposition of the prices was performed based on wavelet analysis. The series was standardized to the unit interval and the decomposition was conducted on each series. ${ }^{56}$ The wavelet basis function used was that of Daubachies 4. To make the number of observation a power of 2 , the data were padded with zeros up to 512. The Nyquist frequency band detail is approximately equal to $\mathrm{Fc} / 2$ to $\mathrm{Fc}$ where $\mathrm{Fc}$ is the Nyquist critical frequency or $1 / 2$ the sampling rate of the input series. In this case the sampling interval is one month so $\mathrm{Fc}$ is $1 / 2$ months. Observations from 257 to 512, which represent approximately half the time interval represents the frequency of $1 / 2$ months to one month. Observations between the range of 129-256 represent 
the next frequency at $1 / 4$ to $1 / 2$ months and so on. Figure 6.23 demonstrates the information context of the outliers, previously discussed in the other diagnostics. The second half the series provides much information to the overall series as would be expected. Notice that in Figure 6.25 , the presence of low frequency play a more prominent role in the beginning of the data series, the 1960s for example, then in later parts of the series. Recall, that to obtain the original series, we must sum each of the seven spectral components for each observation. Thus, we can see graphically the contribution of each sampling interval, rather high or low to the overall data value. This gives a nice advantage over other techniques.

Figure 6.23 Wheat Spectral Components for the Sampling Rate of 1/2 to 1 Month

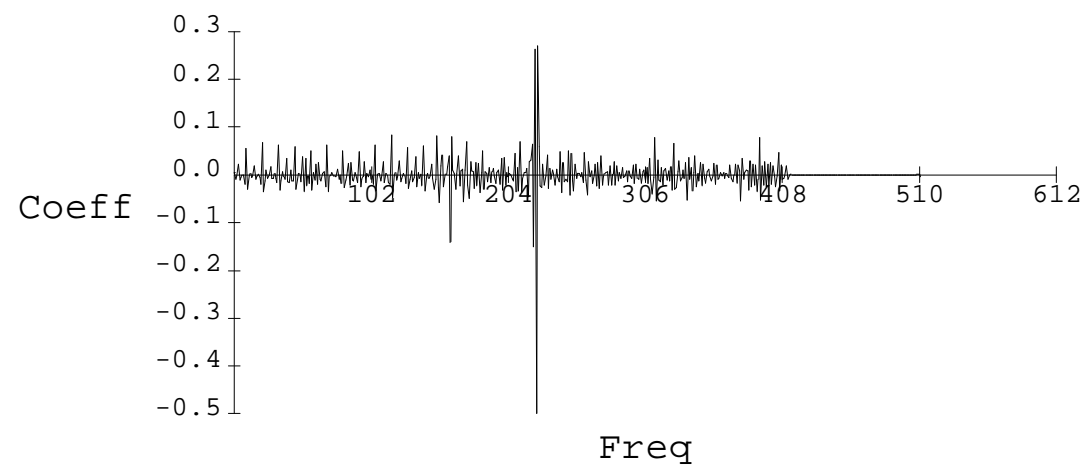

${ }^{56}$ I have only included the results for the wheat price series due to its interesting properties. 
Figure 6.24 Wheat Spectral Components for the Sampling Rate of 1/4 to 1/2 Months

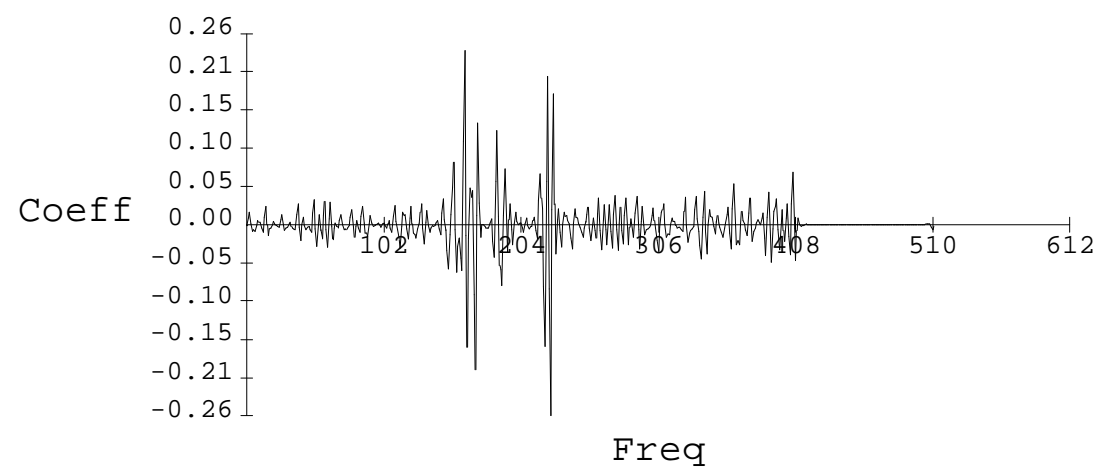

Figure 6.25 Wheat Spectral Components for the Sampling Rate of 1/6 to 1/4 Months

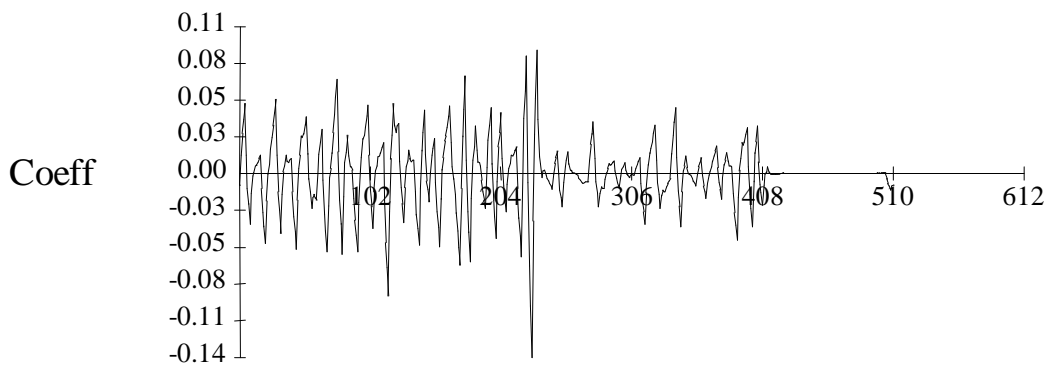

Freq 
Figure 6.26 Wheat Spectral Components for the Sampling Rate of 1/8 to 1/6 Months

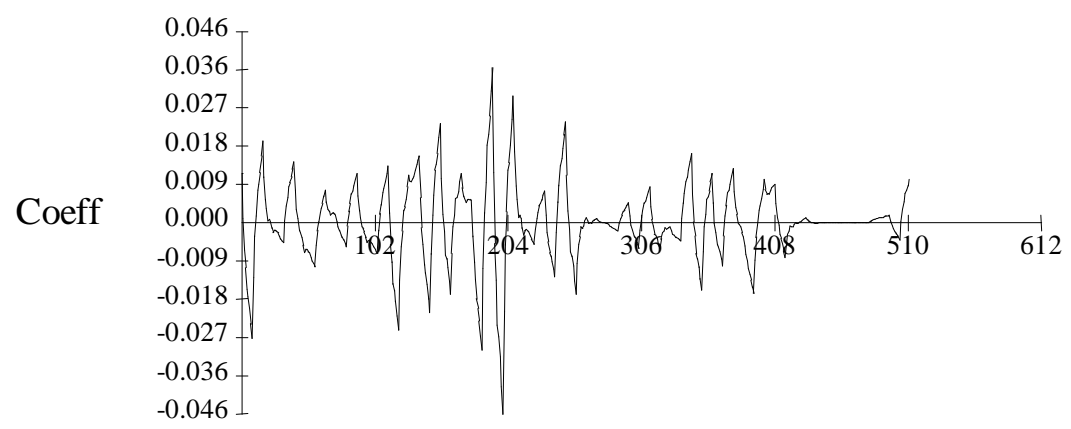

Freq

Figure 6.27 Wheat Spectral Components for the Sampling Rate of 1/10 to 1/8 Months

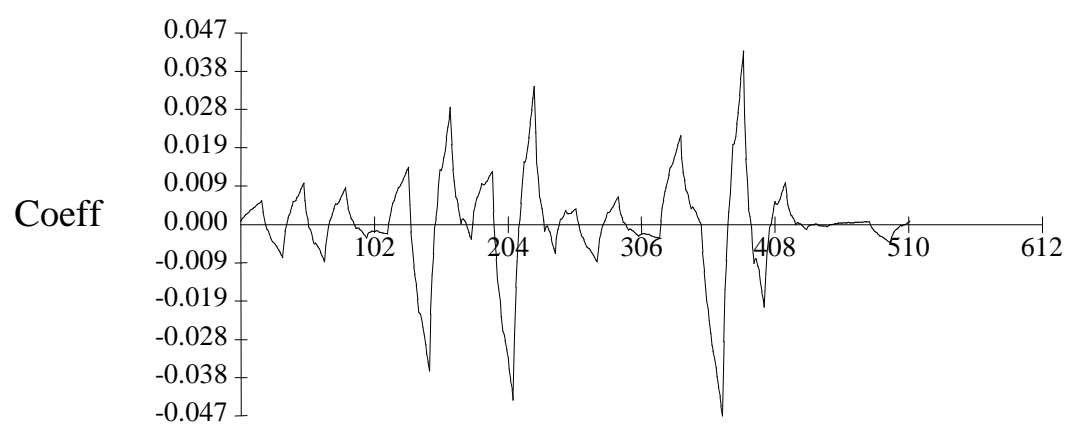

Freq 
Figure 6.28 Wheat Spectral Components for the Sampling Rate of 1/12 to 1/10 Months

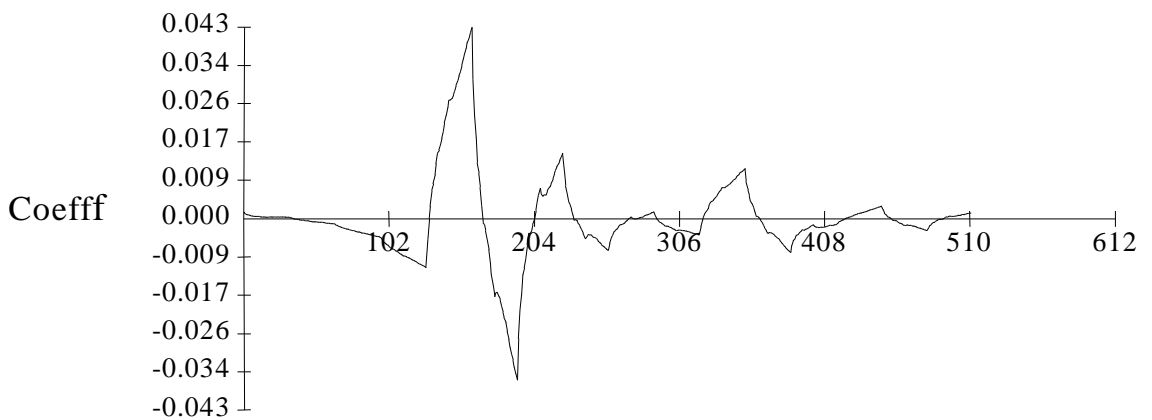

Freq

Figure 6.29 Wheat Spectral Components for the Sampling Rate of 1/14 to 1/12 Months

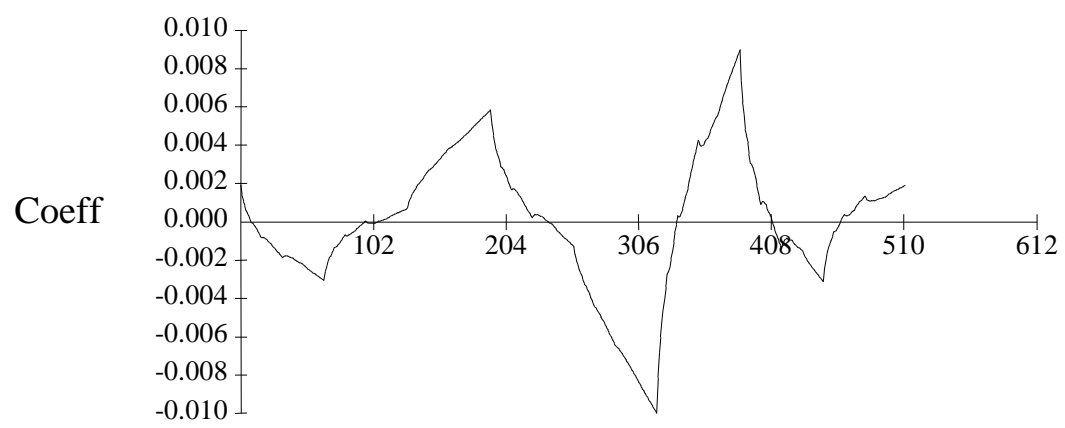

Freq 
Figure 6.30 Wheat Spectral Components for the Sampling Rate of 1/16 to 1/14Months

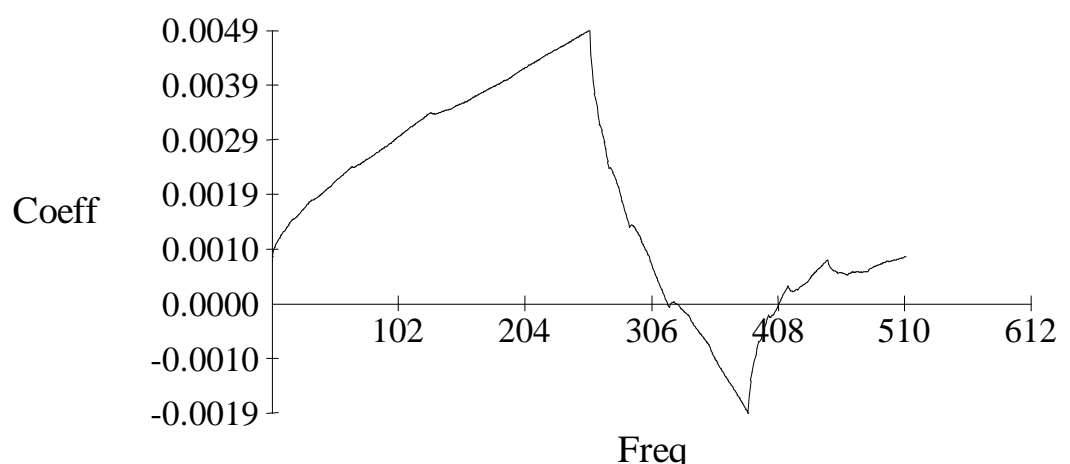

From these graphs, it is easy to see that contributions of the first decade of the data, that is 120 observations for the seasonally adjusted wheat growth rates, have more information content in the first two sampling intervals that at lower frequencies. However, Figures 6.29-6.30 show that as we move into the 1970 s there is some information context that is significant for low frequency components. The power specturm for these growth rates should have a similiar trait. That is, that increases in frequency were similar across all time scales. This makes wheat growth rates appear to have a potential statistical similarity, which would lend itself to a fractal dimension. Thus, we can hypothesize that there is some underlying structure to wheat price growth rates.

While summary statistics provide useful information, it is important to establish the empirical temporal and distributional properties as well. Temporal properties include:

- autocorrelation;

- the presence of "long memory" or persistence(i);

- the presence of the Machina and/or Taylor effects; and

- a determination of the autocorrelations of sign of $r(t)$.

$(6.1)$ 


$$
\begin{aligned}
& (i) \operatorname{corr}\left(\left|r_{t}\right|,\left|r_{t-k}\right|\right)>\operatorname{corr}\left(r_{t}^{2}, r_{t-k}^{2}\right) \\
& (\text { ii }) \operatorname{corr}\left(\left|r_{t}\right|,\left|r_{t-k}\right|\right)>\operatorname{corr}\left(\left|r_{t}\right|^{\theta},\left|r_{t-k}\right|^{\theta}\right) \text { where } \theta \neq 1 \\
& \text { (iii) } \operatorname{corr}\left(\left|r_{t}\right|^{\delta},\left|r_{t}\right|\right)>\operatorname{corr}\left(\left|r_{t}\right|^{\delta},\left|r_{t}\right|^{\theta}\right) \\
& \text { where for any } \delta \text { and } \theta \neq 1
\end{aligned}
$$

Besides the temporal properties alluded to above, there are certain distributional properties that can be obtained from the data. These are:

- the independence of $|r(t)|$ and sign $r(t)$;

- the equality of the mean $|\mathrm{r}(\mathrm{t})|$ and its standard deviation; and

- $|\mathrm{r}(\mathrm{t})|$ has a marginal distribution which is exponential.

In an attempt to examine each one of these temporal and distributional properties, tests were conducted on each series for the existence of the autocorrelation effect, the presence of long memory and presence of the Machina and Taylor effects. Before examining the autocorrelation effect, tests for the Machina and Taylor effects were conducted on each series. Table 6.4 contains the results of these tests. 
Table 6.4 Test for the Taylor Effect on Seasonally Adjusted Growth Rates, $\theta=2$

\begin{tabular}{|c|c|c|c|c|c|c|c|c|c|c|c|c|}
\hline \multirow[b]{2}{*}{ K } & \multicolumn{2}{|c|}{ Bananas } & \multirow{2}{*}{$\begin{array}{l}\text { Beef } \\
\mathrm{r}(\mathrm{t})\end{array}$} & \multirow[b]{2}{*}{$|r(t)|$} & \multicolumn{2}{|l|}{ Coffee } & \multicolumn{2}{|c|}{ Soybean } & \multicolumn{2}{|l|}{ Wool } & \multicolumn{2}{|l|}{ Wheat } \\
\hline & $\mathrm{r}(\mathrm{t})$ & $|r(t)|$ & & & $\mathrm{r}(\mathrm{t})$ & $|r(t)|$ & $\mathrm{r}(\mathrm{t})$ & $|r(t)|$ & $\mathrm{r}(\mathrm{t})$ & $|r(t)|$ & $\mathrm{r}(\mathrm{t})$ & $|r(t)|$ \\
\hline 1 & 0.116 & 0.066 & 0.263 & 0.150 & 0.252 & 0.336 & 0.380 & 0.352 & 0.441 & 0.211 & 0.144 & 0.202 \\
\hline 2 & -0.009 & 0.031 & 0.193 & 0.131 & 0.022 & 0.219 & 0.302 & 0.006 & -0.011 & 0.170 & 0.120 & 0.163 \\
\hline 3 & 0.098 & 0.098 & 0.180 & 0.139 & 0.125 & 0.179 & 0.104 & -0.041 & -0.015 & 0.227 & 0.193 & 0.211 \\
\hline 4 & 0.145 & 0.140 & 0.206 & 0.092 & 0.063 & 0.235 & 0.224 & -0.022 & -0.011 & 0.129 & 0.069 & 0.109 \\
\hline 5 & 0.036 & 0.049 & 0.271 & 0.019 & -0.015 & 0.158 & 0.159 & -0.004 & -0.011 & 0.168 & 0.108 & 0.158 \\
\hline 6 & 0.021 & 0.010 & 0.253 & 0.057 & 0.018 & 0.100 & 0.110 & 0.071 & 0.001 & 0.228 & 0.167 & 0.224 \\
\hline 7 & 0.034 & 0.003 & 0.254 & 0.028 & 0.010 & 0.094 & 0.042 & 0.133 & 0.022 & 0.080 & 0.033 & 0.105 \\
\hline 8 & 0.034 & -0.003 & 0.214 & 0.061 & 0.094 & 0.029 & 0.011 & 0.147 & 0.048 & 0.086 & 0.033 & 0.134 \\
\hline 9 & 0.064 & 0.164 & 0.136 & 0.101 & 0.029 & 0.117 & 0.037 & 0.146 & 0.000 & 0.054 & 0.009 & 0.078 \\
\hline 10 & 0.048 & 0.033 & 0.191 & 0.154 & 0.118 & 0.172 & 0.074 & 0.014 & -0.007 & 0.077 & 0.019 & 0.099 \\
\hline
\end{tabular}

Note: The comparison is between the value of $r(t)$ and $|r(t)|$.

Granger and Ding (1994) point out that if pairs or groups of absolute returns have (approximately) bivariate or multivariate exponential distributions, then nonlinear models are appropriate for forecasting purposes. For example, if $|\mathrm{r}(\mathrm{t})|,|\mathrm{r}(\mathrm{s})|$ have a joint exponential distribution then

$$
E\left(r_{t}^{2 \cdot} \mid r_{t-k}\right)=\beta_{0}+\beta_{1} r_{t-k}^{2}+\beta_{2}\left|r_{t-k}\right|
$$

Table 6.4 shows the results of applications of the distributional tests as well. Notice that growth rates in general, as Ding and Granger (1994) point out, do indeed have unconditional moments that are equal. However, as Table 6.4 shows, they do not have exponential marginal distributions that suggest that long memory of a fractional nature may not be present in the data.

Table 6.4 displays the magnitude of the partial autocorrelation coefficients for each of the seasonally adjusted growth rates, $\mathrm{r}(\mathrm{t})$. The asterisk represents coefficients that are greater than two standard errors. It is apparent from these significance values and the results of the LjungBox tests that linear dependence exists in all of the sample prices. As mentioned in the preceeding chapter, removal of any linear dependence is necessary in order to determine if a 
nonlinear component is present in the series. Since many studies conduct pre-filtering with $\mathrm{AR}(\mathrm{p})$ models, an approach that is adopted here. Any non-linearity that is present is then regulated to the residuals. Of course, the assumption is that the non-linearity is additive. The lag order $\mathrm{p}$ selected was based on the minimization of the AIC criterion. As a check, minimization of what was also conducted by the likelihood ratio test with similar results.

To ensure that linear dependence was removed from the data, a series of diagnostic tests were performed as outlined in Chapter 5. This involved the BDS test for independence. For an epsilon equal to the standard deviation of the residuals from these linear AR models and an embedding dimension between 2 and 8 , Table 6.5 shows that the null hypothesis of independence is rejected at the $1 \%$ and $5 \%$ significance level. 
Table 6.5 Residual Diagnostics for Linear Models of Seasonally Adjusted Growth Rates

\begin{tabular}{|c|c|c|c|c|c|c|}
\hline BDS & BANANAS & BEEF & COFFEE & SOYBEAN & WHEAT & WOOL \\
\hline \multicolumn{7}{|l|}{ M } \\
\hline 2 & 1.035 & 4.6362 & 3.63 & 6.34 & 6.75 & 3.31 \\
\hline 3 & 1.35 & 5.84 & 4.83 & 7.10 & 7.14 & 3.31 \\
\hline 4 & 1.84 & 7.08 & 6.07 & 7.73 & 6.57 & 3.95 \\
\hline 5 & 2.58 & 8.16 & 7.08 & 8.22 & 5.90 & 4.14 \\
\hline 6 & 3.09 & 9.44 & 7.85 & 8.34 & 5.68 & 4.51 \\
\hline 7 & 3.45 & 11.05 & 8.62 & 8.73 & 5.98 & 5.28 \\
\hline 8 & 3.66 & 11.17 & 10.02 & 9.06 & 6.47 & 5.81 \\
\hline 9 & 3.95 & 11.29 & 11.50 & 9.26 & 6.76 & 6.20 \\
\hline 10 & 4.23 & 10.39 & 13.42 & 9.52 & 7.02 & 6.67 \\
\hline Epsilon & .1425 & .1237 & .1093 & .0806 & .0559 & .0805 \\
\hline \multicolumn{7}{|l|}{ LM } \\
\hline 1 & 1.186 & 4.196 & 0.486 & 1.340 & 0.265 & 1.511 \\
\hline 6 & 6.395 & 26.77 & 10.903 & 5.524 & 2.822 & 4.707 \\
\hline 12 & 23.38 & $33.68 * *$ & 14.016 & 12.198 & 17.11 & 7.15 \\
\hline Lbe(18) & 5.63 & 23.52 & 15.69 & 17.32 & 31.33 & 13.88 \\
\hline Skewness & -0.2257 & .0718 & 0.412 & -0.126 & 0.845 & 0.908 \\
\hline Kurtosis & 3.788 & 4.964 & 5.938 & 12.560 & 36.50 & 15.38 \\
\hline Jarque-Bera & $13.71^{* *}$ & 64.67 & 154.82 & 1525.42 & 1875.8 & 2612.86 \\
\hline \multicolumn{7}{|l|}{$\mathrm{ARCH}$} \\
\hline 1 & 0.004 & $30.61 * *$ & $8.05 * *$ & $19.75^{* *}$ & $77.03^{* *}$ & 3.55 \\
\hline 6 & $14.56^{* *}$ & $50.42 * *$ & 32.84 & 86.76 & $104.32 * *$ & $0.77 * *$ \\
\hline 12 & $32.47 * *$ & $53.52 * *$ & $36.89 * *$ & $93.00 * *$ & $104.59 * *$ & $21.77 *$ \\
\hline
\end{tabular}

NOTE: ** Significant at the $1 \%$ level

* Significant at the $5 \%$ level

However, one can infer from the results of the BDS tests that since the linearity was accounted for in the transformed prices, there exists some form of nonlinearity in the residuals. To ascertain if linearity was present, the Lagrangian Multiplier test for serial correlation was 
performed as well as the Ljung-Box test for serial dependence. For both tests and across all transformed prices, the null hypotheses of no serial dependence are accepted. It is also quite evident that the residuals are quite leptokurtic and non-normal.

In addition to the above diagnostics, the ARCH test was performed. As can be seen from the Table 6.6, significant $\mathrm{ARCH}$ effects were present for all filtered growth rates. This is not surprising because of the a priori belief that the seasonally adjusted growth rates were not covariance stationary. While heteroscedasticity is present in the second moment of the prices series, the form is not yet identifiable. For example, an increasing variance could be present in the form of a multiplicative non-linearity. Therefore, it is quite possible that this could be attributed to the feature of additive nonlinearity such as the logistic as opposed to the variance nonlinearity of the ARCH models. All other transformed price series exhibit significant ARCH effects.

Complementary to the tests presented in Table 6.6, Table 6.7 presents the results of the fourth testing step, based on Heist's (1989) third moment test. 
Table 6.6 Third Moment Test for Linearly Filtered Residuals

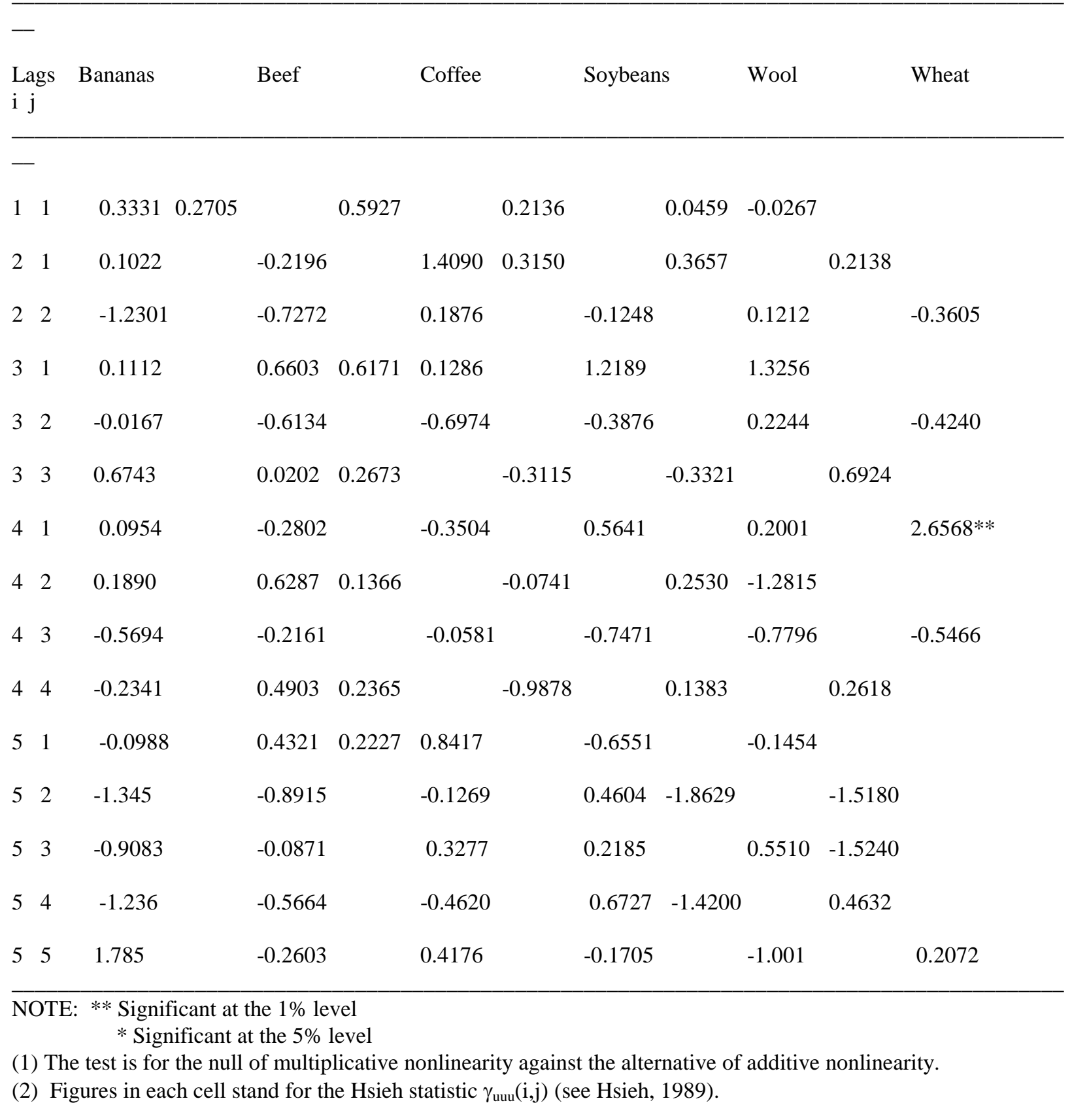

One can conclude that for the lags of 4 and 1 , the null hypothesis of variance nonlinearity was rejected for wheat prices. That is, for wheat growth rates, there is some dependence in the third 
moment of the prices series. Although, this could be considered an outlier to the results, the preliminary visual inspection of the data lends support to this belief.

This provides some evidence that wheat price growth rates may behave differently from that of the other commodities. This suggests that the residuals are quite possibly independent. Given the strong presence of the ARCH effect or variance nonlinearity in the time series, both ARCH and GARCH(1,1) models were fitted to the growth rates. The specification of the conditional mean for these models is the same as for the other growth rates. Table 6.8 shows that all models pass the fourth moment existence conditions as well as conditions for stationary.

Table 6.7 GARCH $(1,1)$ and ARCH (3) Model Estimates for the Conditional Variance

\begin{tabular}{|c|c|c|c|c|c|c|}
\hline & Bananas & Beef & Coffee & Soybeans & Wool & Wheat \\
\hline \multicolumn{7}{|l|}{-} \\
\hline $\begin{array}{l}\text { Parameter } \\
\alpha(0)\end{array}$ & $\begin{array}{l}0.0126 \\
(9.65)\end{array}$ & $\begin{array}{l}0.0009 \\
(2.14)\end{array}$ & $\begin{array}{l}0.0031 \\
(2.46)\end{array}$ & $\begin{array}{l}0.0027 \\
(3.06)\end{array}$ & $\begin{array}{l}0.0039 \\
(3.07)\end{array}$ & $\begin{array}{l}0.0038 \\
(9.53)\end{array}$ \\
\hline$\alpha(1)$ & $\begin{array}{c}-0.0576 \\
(-10.92)\end{array}$ & $\begin{array}{l}0.1875 \\
(3.63)\end{array}$ & $\begin{array}{l}0.1825 \\
(3.665)\end{array}$ & $\begin{array}{l}0.2819 \\
(4.24)\end{array}$ & $\begin{array}{l}0.2598 \\
(3.60)\end{array}$ & $\begin{array}{l}0.124 \\
(1.664)\end{array}$ \\
\hline$\alpha(2)$ & $\begin{array}{l}0.1012 \\
(1.67)\end{array}$ & & & & & \\
\hline$\alpha(3)$ & $\begin{array}{l}0.0533 \\
(1.05)\end{array}$ & & & & & \\
\hline$B(1)$ & & $\begin{array}{l}0.769 \\
(13.32)\end{array}$ & $\begin{array}{l}0.7579 \\
(13.04)\end{array}$ & $\begin{array}{l}0.658 \\
(10.78)\end{array}$ & $\begin{array}{l}0.6456 \\
(8.469)\end{array}$ & $\begin{array}{l}0.0734 \\
(2.034)\end{array}$ \\
\hline $\begin{array}{l}\text { Fourth mt. } \\
\sum \alpha(\mathrm{i})+\beta(\mathrm{i}) \\
\log \end{array}$ & 0.109 & 0.9565 & 0.9404 & 0.9399 & 0.9054 & 0.1974 \\
\hline Likelihood & 626.05 & 691.39 & 553.29 & 600.75 & 626.06 & 569.78 \\
\hline
\end{tabular}


NOTE: Existence of the fourth moment requires that the estimated value of the expression given by Bollerslev (1987) be less than one. Stationarity in the variance requires that the sum of the coefficents be between -1 and 1 . Asymptotic standard errors are in parentheses.

An ARCH (3) model was selected for coffee due to the non-convergence of the GARCH $(1,1)$ specification. The selection of the $\operatorname{GARCH}(1,1)$ specification was chosen for the other commodity prices, since it provided a better fit to the data than the $\mathrm{ARCH}(3)$ specification. Table 6.9 provides diagnostics of the standardized residuals of these specifications. Notice that only transformed wheat prices reject the null of the BDS test. This suggests that some structure may still exist in the data. Given the test procedure outlined in the previous section, the evidence suggests that growth rates of both wheat and rice prices are suitable candidates to test for chaotic behavior. 
Table 6.8 Residual Diagnostics from ARCH and GARCH Models

\begin{tabular}{crrrrrr}
\hline BDS & Bananas & \multicolumn{1}{l}{ Beef } & \multicolumn{1}{l}{ Coffee } & \multicolumn{1}{l}{ Soybean } & Wool & Wheat \\
M & & & & & & \\
2 & 1.403 & -.627 & 1.474 & .393 & 1.158 & -.316 \\
3 & 1.025 & 3.34 & 1.428 & .442 & 3.098 & -1.019 \\
4 & 1.312 & 5.84 & 1.454 & .546 & 3.271 & -.653 \\
5 & 1.805 & -4.85 & 1.256 & .559 & 3.061 & -.626 \\
6 & 1.946 & -3.32 & .849 & .307 & 3.191 & -.580 \\
7 & 2.011 & -2.39 & .734 & -.020 & 3.82 & -.283 \\
8 & 2.022 & -1.78 & 1.08 & -.317 & 4.49 & -.138 \\
9 & 2.000 & -1.36 & 1.504 & -.722 & 4.82 & -.188 \\
10 & 2.154 & -1.06 & 2.133 & -.691 & 5.27 & -.195 \\
SD/Spread & .1354 & .01357 & .1262 & .1280 & .0721 & .08621 \\
Qe(18) & 6.15 & 16.10 & 26.29 & 23.45 & 26.91 & 32.39 \\
Skewness & -0.179 & -0.103 & .109 & .924 & 3.61 & -1.378 \\
Kurtosis & 3.915 & 3.916 & 5.08 & 4.78 & 38.85 & 15.92 \\
Jarque-Bera & $16.11 * *$ & $14.70 * *$ & $73.31 * *$ & $186.17 * *$ & $22298.0 * *$ & $2909.58 * *$ \\
& & & & & & \\
\hline
\end{tabular}

**Significant at the $1 \%$ level.

This latter commodity was found suitable to evaluate for chaotic behavior, the indicated fifth step of analysis. Table 6.10 displays the estimates of the correlation and Lyapunov exponents for the residuals from the previous testing steps. For the sake of accuracy and consistency, residuals from the GARCH and neural network models as well as the shuffled residual test to determine the relative magnitude of both the correlation and Lyapunov exponent is performed. 
Table 6.9 The Root Mean Square Error Forecast Statistics for AR, ARCH-GARCH, Neural Network and Nearest Neighbor Models For Forecast From June, 1993 To June 1994.

\begin{tabular}{|c|c|c|c|c|c|c|}
\hline & Bananas & Beef & Coffee & Soybeans & Wool & Wheat \\
\hline \multicolumn{7}{|c|}{ AR MODELS } \\
\hline \multirow[t]{2}{*}{ Order } & 10 & 11 & 9 & 1 & 4 & 1 \\
\hline & 0.173 & 0.043 & 0.086 & 0.064 & 0.040 & 0.045 \\
\hline \multicolumn{7}{|c|}{ ARCH-GARCH } \\
\hline & 0.162 & 0.039 & 0.082 & 0.061 & 0.037 & 0.044 \\
\hline \multicolumn{7}{|c|}{ NEURAL NETWORKS } \\
\hline GRNN & 0.1972 & 0.048 & 0.092 & 0.060 & 0.044 & 0.043 \\
\hline NN & 0.1573 & 0.039 & 0.085 & 0.060 & 0.045 & 0.039 \\
\hline RNN1 & 0.1856 & 0.040 & 0.077 & 0.061 & 0.044 & 0.038 \\
\hline RNN2 & 0.1566 & 0.040 & 0.076 & 0.060 & 0.047 & 0.046 \\
\hline RNN3 & 0.1577 & 0.046 & 0.075 & 0.060 & 0.045 & 0.045 \\
\hline \multicolumn{7}{|c|}{ WAVELET-NEURAL NETWORK } \\
\hline & 0.1567 & 0.038 & 0.071 & 0.055 & 0.066 & 0.031 \\
\hline \multicolumn{7}{|c|}{ NEAREST NEIGHBOR } \\
\hline \multicolumn{7}{|c|}{ Euclidean } \\
\hline Metric & 0.1222 & 0.039 & 0.0424 & 0.060 & 0.0639 & 0.0426 \\
\hline \multicolumn{7}{|c|}{ “City Block" } \\
\hline Metric & 0.1219 & 0.043 & 0.0393 & 0.058 & 0.0631 & 0.04 \\
\hline
\end{tabular}

Note: The WAVELET-NEURAL NETWORK Model was accomplished by using a $10 \%$ threshold to remove those coefficients in the decomposition that were close to zero in terms of their contribution in both time and frequency. The Neural Network employed was simple backpropagation with the the AR specification for each input neuron coupled with 25 neurons in the hidden layer. The GNN,NN and the three recurrent neural network structures RNN1, RNN2, and RNN3 invoked similiar methodology

It is apparent that although neither growth rate saturates, wheat growth rates do increase at a slower rate even at dimensions above eight. For the rice growth rates, the evidence suggests that there might be some structure in the residuals; however, it would consist of a high dimensionality 
(i.e. embedding dimensions greater than 5) which is impossible to examine given the sample limitation imposed by the data set on the power of the tests employed.

Filtered wheat prices, however, appear to possess a nonlinear structure with a dimensionality around four. The Lyapunov exponent was estimated using the Wolf et al. (1985) algorithm. By varying only the evolution time, it appears that a positive Lyapunov exponent does exist. However, as one can see from the Table the point estimates from these exponents do not demonstrate consistent positive behavior as a function of the evolution time. As mentioned previously, the explosive nature of the prices can be related to the fact that these prices are near unit root in the variance as opposed to the mean that can account for the positive magnitude of the Lyapunov exponent.

The shuffle tests on the ARCH and GARCH residuals confirm the earlier shuffle test results. All shuffled series of the ARCH-GARCH residuals are higher than the corresponding correlation dimension estimate. This provides strong evidence for the proposition that there is some nonlinear process with a deterministic component underlying the series. 
Table 6.10 Correlation Dimension and Largest Lyapunov Exponent Estimation Results for Wheat Growth Rates

\begin{tabular}{lclll}
\hline $\begin{array}{l}\text { Correlation } \\
\text { Dimension Estimate } \\
\mathrm{m}\end{array}$ & GARCH Residuals & NN Residuals & Wavelet-NN & Shuffled GARCH \\
& & & & \\
2 & & & & \\
& 0.860 & 0.756 & 1.223 & 0.345 \\
3 & $( \pm 0.078)$ & $( \pm 0.103)$ & $( \pm 0.154)$ & $( \pm 0.074)$ \\
& 1.455 & 1.566 & 1.455 & 1.024 \\
4 & $( \pm 0.112)$ & $( \pm 0.097)$ & $( \pm 0.176)$ & $( \pm 0.065)$ \\
& 2.742 & 3.122 & 1.987 & 1.987 \\
5 & $( \pm 0.146)$ & $( \pm 0.125)$ & $( \pm 0.112)$ & $( \pm 0.121)$ \\
& 2.892 & 4.198 & 2.968 & 2.568 \\
6 & $( \pm 0.161)$ & $( \pm 0.231)$ & $( \pm 0.124)$ & $( \pm 0.154)$ \\
& 3.620 & 5.234 & 4.125 & 3.678 \\
7 & $( \pm 0.149)$ & $( \pm 0.198)$ & $( \pm 0.169)$ & $( \pm 0.064)$ \\
& 4.194 & 6.345 & 5.798 & 3.988 \\
8 & $( \pm 0.108)$ & $( \pm 0.098)$ & $( \pm 0.091)$ & $( \pm 0.210)$ \\
& 4.205 & 6.981 & 6.112 & 4.125 \\
& $( \pm 0.068)$ & $( \pm 0.121)$ & $( \pm 0.137)$ & $( \pm 0.165)$
\end{tabular}

LYAPUNOV EXPONENT ESTIMATE

Evolution Time

$\begin{array}{lclll}2 & 0.0851 & -0.101 & -0.122 & -0.002 \\ 3 & (0.020) & (0.04) & (0.09) & (0.03) \\ & 0.1071 & 0.005 & -0.056 & 0.001 \\ 4 & (0.0301) & (0.025) & (0.10) & (0.04) \\ & 0.0669 & -0.135 & 0.003 & 0.002 \\ 5 & (0.0345) & (0.029) & (0.08) & (0.046) \\ & 0.0646 & 0.0432 & 0.001 & 0.031 \\ 6 & (0.0561) & (0.025) & (0.016) & (0.056) \\ & 0.0741 & 0.0349 & -0.003 & 0.001 \\ & (0.0612) & (0.065) & (0.023) & (0.067)\end{array}$

NOTE: The value of epsilon was taken to be the standard deviation of each filtered series. The term in the parentheses represents a 95\% confidence interval around the regression coefficient. The parameter values for the Lyapunov exponent is minimum scale $=0.0055$ and the maximum scale $=0.1200$, the embedding dimension was chosen to be 4 .

To confirm the results, a test outlined by Brock and Baek (1991) was performed on the transformed wheat residuals. The null hypothesis that seasonally adjusted growth rates could be generated by an IID stochastic process is rejected. Eckman and Ruelle (1985) state that the 
$\mathrm{K}(\mathrm{e}, \mathrm{m})$ is a lower bound to the true Kolmogorov entropy, and positive Kolmogorov entropy is associated with chaos. These results suggest that there is something to be gained by using the historical series to forecast future wheat prices.

A noise explanation of the data, as well as linear structure plus noise explanation can be rejected. Thus the hypothesis of nonlinear structure is accepted. As demonstrated in Table 6.10, neural network and nearest neighbor forecasts were superior to the ARCH-GARCH models. Because non-linearity enters into the specification through the conditional variance that is difficult to specify, neural network models may be more advantageous in terms of forecast error then uncovering the correct parametric form of the variance process.

Table 6.11 Kolmogorov Entropy Estimates for Wheat Price Growth Rates

\begin{tabular}{llll}
\hline $\begin{array}{l}\text { Entropy } \\
\text { Estimate }\end{array}$ & \multicolumn{2}{c}{ GARCH Residuals } \\
& Entropy & ASE $/ \sqrt{T}$ & \\
$\mathrm{~m}$ & & & TEST \\
1 & 0.3164 & 0.080 & -8.51 \\
2 & 0.3059 & 0.083 & -9.52 \\
3 & 0.3152 & 0.087 & -9.59 \\
4 & 0.3245 & 0.095 & -9.79 \\
5 & 0.3139 & 0.094 & -9.88 \\
6 & 0.2853 & 0.097 & -9.34 \\
& & & \\
& & Wavelet-NN Residuals & \\
& & & ASE $/ \sqrt{T}$ \\
& & & -10.85 \\
1 & Entropy & 0.101 & -10.84 \\
2 & 0.557 & 0.091 & -10.95 \\
3 & 0.579 & 0.093 & -10.92 \\
4 & 0.624 & 0.089 & -11.04 \\
5 & 0.587 & 0.091 & \\
6 & 0.455 & 0.121 & \\
& 0.689 & & \\
\hline
\end{tabular}

Note: The null hypothesis is that filtered wheat prices are generated by an iid process. 
Finally, in Table 6.11 we have the results of the entropy measures. Notice that we can reject the null hypothesis that wheat prices are white noise. The results are independent of embedding dimensions and both functional form specifications. We can conclude that there is some form of non-linear process that generates wheat prices that could be indeed chaotic. 


\section{CHAPTER 7: SUMMARY AND CONCLUSIONS}

The historian is a prophet looking backwards. -August von Schlegel

\section{Introduction}

This study has evaluated the nonlinear price behavior of selected agricultural commodities traded on international markets. To provide a framework with which to discuss the results of this work, a recap of each one of the objectives is presented.

\section{A Recap of Objectives}

1. To provide a review of current as well as past attempts to model agricultural prices, most notably, commodity prices. This review is to not only consist of past attempts at modeling agricultural prices and also contrast them with the basic tenets of chaos theory.

The review of the literature from both an empirical and theoretical perspective demonstrates the applicability of nonlinear time series methods to modeling commodity prices. Although rather restrictive with their scalar emphasis and extrapolations to chaos theory, this study added to the literature by integrating the techniques inherent in the previous studies into this one.

While an important step in the right direction, several additional unresolved theoretical issues remain. The influence of neoclassical theory and equilibrium dynamics into the modeling of agricultural prices needs to be examined along with traditional assumptions of linearity and negative feedback coupled with diminishing returns.

2. To demonstrate on a theoretical level, the necessary conditions for agricultural markets to exhibit chaotic behavior. This is to be completed by formulating a competitive market model for agricultural commodities and then determining any necessary restrictions to be enforced on the parameters and functional forms of the model. 
The attempt to pigeon hole agricultural prices into matchbook models such as the logistic and tent map specifications, which have topologically similar properties needs to be further addressed. Higher dimensional analysis as an extension to the work here is needed to further examine issues related to backward bending supply curves and multiple demand curves. Revisions of standard theoretical assumptions in light of empirical evidence has to this point not readily have been forthcoming. This analysis suggests that chaotic behavior may occur in the wheat market has to be further investigated in a theoretical sense, given due to the subtle characteristics that are unique to this market and the producing and supplying regions.

3. To examine the current methodology for the procedures used in the detection of chaotic behavior. Also, to suggest possible improvements where necessary and develop the testing framework into a logical and coherent one.

While the testing methodology presented in this analysis is typical of time series analyses, there are some problems in testing for chaotic behavior. One is with respect to the lack of asmpytotic results concerning the Lyapunov exponents. A formal hypothesis testing procedure that takes into account the modeling process under the null, could greatly add to determining the power properties of the test. As it stands now, the exponent is little more than a statistical artifact often employed in an arbitrary fashion. In this study, bootstrapping was done in order to develop standard errors for the exponents. This was one of the first works that attempted such a feat and it is recognized that some of the underlying assumptions concerning bootstrapping may have been violated.

Neural network methods as an improved filtering technique for assessing the amount of nonlinear structure in the data should be useful in the immediate future, especially when coupled 
with wavelet decompositions. The ten percent thresholding of the wavelet coefficients did lead to a better fit overall than other specifications. The use of the recurrent models along with the wavelet models as applied to nonstationary data, as done in this study, was found to be superior in terms of RMSE to the linear and/or GARCH models. Clearly, more work is needed in this area. However, this was the first empirical study to use the neural network in combination with wavelet analysis as an approach in the determination of chaotic behavior.

4. Using the testing framework developed under objective 3, to test for the presence of chaotic behavior in a sample of internationally traded agricultural commodity prices.The sampling interval for these prices was monthly, obtained over the period 1960-1994. The commodities selected were banannas, beef, coffee, soybeans, wool and wheat.

After the rigors of testing were accomplished, it was determined that none of the series unequivocally exhibited chaotic behavior. While wheat prices may to some degree, there is no doubt that GARCH modeling of these prices has considerable influence. However, given the improve fit by neural and other non-parametric methods, it can be concluded that GARCH models with their associated conditional means and variances is just a second-best way to account for non-stationarities in the unconditional distribution of prices.

Some of the stardard assumptions invoked in this GARCH analysis were:

- Growth rates are stationary

- All tests employed are uniformally most powerful

- The embedding range and epsilion selected for the dimension analysis are robust

- The BDS tests were robust in the presence of several non-linear alternatives

- The assumptions for the lyapunov exponents are robust

- Optimal identification of lag structure for AR and neural methods are achieved 
The hypothesis of low dimensional chaos is not supported by Brock's residual test. Estimated dimensions for rescaled data are either larger than those for the raw data, or estimates for both series are increasing with embedding dimensions. These results favor the stochastic approach when determining the generating process. But, the results, using the preferred BDS test statistic, are mixed. The GARCH residuals are not iid for some of the commodities, which is consistent with deterministic chaos.

The GARCH $(1,1)$ process is not a perfect model for the data. The results show strong support for the existence of conditional heteroscedasticity, and also show support for the GARCH model, but it is not totally adequate. The neural network models outperformed the ARCH-GARCH models in terms of forecasting error. The results do not provide strong support for deterministic chaos.

The primary insight from Mandelbrot's work is that the distributions of prices are approximately scale invariant; it does not matter from the stochastic point of view if you look at them by the minute or by the year. The results of this work seem to lend support to this viewpoint.

Overall, a sequential testing methodology has been adopted and has helped in identifying the underlying price generating processes. The empirical results confirm regular nonlinear dependence for growth rates in bananas, beef, coffee, soybeans, wool and wheat. The results for wheat did not confirm strong nonlinear dependence based on the ARCH effect. Although linear dependence was confirmed for growth rates of rice prices, tests of the Lyapunov exponent suggests possible chaotic behavior for wheat growth rates. 
These results complement the findings of Labys and Granger (1970) for an earlier period; they found some dependence in spot and future series over and above confirmation of the random walk model, not only for wheat but also for rye and soybeans and cottonseed that includes both meal and oil. None of the agricultural price studies cited in the first part of this paper deal with monthly prices and so comparisons cannot be made at this frequency cited earlier. Further analysis with equivalent data sets would be useful in this respect. And more robust test methods are likely to be available to advance the present analysis in this respect.

Overall, an attempt was made to address Tomek's (1994) suggestions for improved empirical research. He contends that there are four necessary conditions for improved empirical research:

- Higher quality data

- Improved models

- Use of appropriate estimators

- More thorough evaluations

Throughout this thesis these types of considerations were entertained.

\section{Implications for Forecasting}

There are several implications to be gained from this work. The first is in regard to wheat prices, which suggests that there is some form of additive nonlinear operator at work in the price series. Although it may be inherently a nonstationarity in the variance and the explosive nature of the processes suggest that is certainly probable, it does reflect the need to treat wheat prices differently with respect to the modeling process. While the other prices examined in this study had some linear structures coupled with the non-stationairty in the variance, adequate filtering techniques were able to remove their influence. 
The second implication is that parametric formulations that attempt to build forecasting models from matchbook models and then derive the parameter specifications for chaotic behavior are too simplistic to represent these data series, especially for wheat. More theory in the area of expectations and the role of future prices along with inventory behavior needs to be examined and properly modelled for better forecasting performance. The results of this work also do suggest limitations to the modeling of univariate price series.

\section{A Final Comment}

One of the main difficulties inherent in this and other similar works has been stated by Ramsey et al. (1990) that

"In the problems examined to date in physics and chemistry, the simple dichotomy of: either an attractor or the data are merely (high dimensional) noise' has been considered to be appropiate. But this is not the case in economics. The extended maintained hypothesis must include as alternatives the options that the data come from ARIMA or nonlinear stochastic process." (p.994)

The evidence of structure in agricultural prices raises further questions about the efficient markets hypothesis, because of the probability that nonlinear models can add to monthly prediction precision. A noise explanation of the data as well as linear structure plus noise explanation is rejected. Thus, the hypothesis that a nonlinear structure is present is accepted. As far as chaos is concerned, if the null hypothesis is that of chaos of a type that is deterministic is present, that null hypothesis would have to be cautiously rejected. However, there is no doubt that a form of white chaos may exist in the prices. An important area of future research is to further develop theoretical equilibrium models of agricultural markets that can generate dynamic modeling of the growth rates. Although the conclusions reached here are not encouraging, they do deal with issues of clear importance. The assumption that all systems lead to a state of equilibrium has been dealt the strongest challenge when evaluating the true dynamics of 
agricultural pricing systems. Chaos theory proves that simple systems can actually lead to very complex processes that may appear random but can no longer be discounted as such. Therefore, one should continue to determine the nonlinear dynamical nature of the price series and seek those parameters along with their associated probabilities that can lead to a determination of whether chaotic behavior exists. 


\section{REFERENCES}

Akaike, H. 1969. Fitting Autoregressions For Prediction. Annals of the Institute of Statistical Mathematics 21:243-247.

Akaike, H. 1970a. Autoregressive Model Fitting For Control. Annals of the Institute of Statistical Mathematics 22:163-180.

Akaike, H. 1970b. A Fundamental Relation Between Predictor Identification And Power Spectrum Estimation. Annals of the Institute of Statistical Mathematics 22:219-223.

Akaike, H. 1973. Information Theory And An Extension Of The Maximum Likelihood Principle, in 2nd International Symposium on Information Theory, ed. B.N. Petrov and F. Csaki, pp.267281. Budapest:Akademiai Kiado.

Akaike, H. 1974. A New Look At Statistical Model Identification. IEEE Transactions on Automatic Control 19:716-723.

Akaike, H. 1979. A Bayesian Extension Of The Minimum AIC Procedure. Biometrika 66:23742.

Albers, D. and G. Alexanderson 1985. Mathematical People: Profiles and Interviews New York: Contemporary Books.

Allen, P.G. 1994. Economic Forecasting in Agriculture, International Journal of Forecasting 10:81-135.

Anderson, T.W. 1963. Determination Of The Order Of Dependence In Normally Distributed Time Series. Proceedings of the Symposium of Time Series Analysis ed. M. Rosenblatt p.42546, New York:Wiley.

Anderson, T.W. 1971. The Statistical Analysis of Time Series. New York: John Wiley and Sons, Inc.

Ardeni, P.G. 1989. Does the law of one price really hold for commodity prices? American Journal of Agricultural Economics, 71:661-669.

Arjoon, S. 1993. Non-Parametric Modelling and Inference: An Alternative, Social and Economic Studies 42:209-224.

Arstien, Z. 1983. Irregular Cobweb Dynamics, Economic Letters 11:15-17. 
Bachieler, L. 1900. Theory of Speculation in P. Cootner (ed.) The Random Character of Stock Market Prices Cambridge: MIT Press.

Barnett, W. and P. Chen 1988. The Aggregation-Theoretic Monetary Aggregates are Chaotic and Have Strange Attractors, in W. Barnett, E. Brendt, and H. White, eds. Dynamic Econometric Modeling, Cambridge University Press, New York.

Barnett, W. and P. Chen 1988. Determinstic Chaos and Fractal Attractors as Tools for Nonparametric Dynamical Econometric Inference: With an Application to Divisa Monetary Aggregates, Mathematical Computation Modeling 10:275-296.

Bartels, R. 1982. The Rank Version Of Von Neumann's Ratio Test For Randomness. Journal of the American Statistical Association 77:40-6.

Bauer, R.M.M.J, F.G.M.C. Nieuwland, W. F. C. Verschoor, 1994. German Stock Market Dynamics, Empirical Economics 4:397-418.

Baumol, W.J. and J. Benhabib 1989. Chaos: Significance, Mechanism, And Economic Applications. Journal of Economic Perspectives 3:77-105.

Baumol, W.J. and R.E. Quandt 1985. Chaos Models and their Implications for Forecasting, Eastern Economic Journal 11:3-15.

Benhabib, J. and R.H. Day 1980. Erratic Accumulation, Economic Letters, 6:113-117.

Benhabib, J. and K. Nishimura 1979. The Hopf Bifurcation And The Existence And Stability Of Closed Orbits In Multisector Models Of Optimum Economic Growth. Journal of Economic Theory 21:421-444.

Bera, A.K. and C.M. Jarque 1981. An Efficient Large Sample Test For Normality Of Observations And Regression Residuals. Australian National University working papers in Econometrics No. 40 Canberra.

Beylkin, G. R., R. Coifman, I. Daubechies, S. Mallat, Y. Meyer, L. Raphael, and B. Rushai (eds) 1992. Wavelets and their Applications, Cambridge Mass. Jones and Bartlett.

Bhansali, R.J. and D.J. Downham 1977. Some Properties Of Autoregressive Model Selected By A Generalization Of Akaike's EPF Criterion. Biometrika 64:547-551.

Bhargava, A.S. 1986. On The Theory Of Testing For Unit Roots In Observed Time Series. Review of Economic Studies 53:369-384.

Blank, S.C. 1991. 'Chaos” in Futures Markets? A Nonlinear Dynamical Analysis, The Journal of Futures Markets, 11:721-728. 
Bollerslev, T. 1988. On The Correlation Structure For The Generalized Autoregressive Conditional Heteroskedastic Process. Journal of Time Series Analysis 9:121-131.

Bollerslev, T. 1986. Generalized Autoregressive Conditional Heteroskedasticity. Journal of Econometrics 31:307-27.

Box, G.E.P. and G.M. Jenkins 1976. Time Series Analysis: Forecasting and Control. Revised edition. Holden-Day San Francisco, CA.

Box, G.E.P. and Pierce, D.A. 1970. Distribution Of Residual Autocorrelations In Autoregressive Moving Average Time Series Models. Journal of the American Statistical Association 65:150926.

Bressler, R.G. and R.A. King, 1970. Markets, Prices, and Interregional Trade. New York: John Wiley and Sons: Chapter 11.

Briggs, K. 1990. An Improved Method for Estimating Lyapunov Exponents of Chaotic Time Series, Physics Letters A 151:27-32.

Brock, W.A. 1987. Notes on nuisance parameter problems in BDS type tests for IID. University of Wisconsin, Madison working paper. Revised November 1989.

Brock, W.A. and E.G. Baek 1991. Some Theory Of Statistical Inference For Nonlinear Science. Review of Economic Studies. 58:697-716.

Brock, W.A., Dechert, W. and J. Scheinkman 1986. A Test Of Independence Based On The Correlation Dimension. SSRI Report \#8702, Department of Economics, University of Wisconsin, University of Houston, and University of Chicago.

Brock, W.A., Hsieh, D.A. and B. LeBaron 1991. Nonlinear Dynamics, Chaos and Instability. Cambridge, MA: The MIT Press.

Brock, W.A. and E.G. Baek 1991. Some Theory Of Statistical Inference For Nonlinear Science. Review of Economic Studies 58:697-716.

Brockett, R.W. 1976. Volterra Series And Geometric Control Theory. Automatica 12:167-76.

Brockwell, P.J. and R.A. Davis 1987. Time Series: Theory and Models, Springer-Verlag:New York.

Burton, M. 1993. Some Illustrations Of Chaos In Commodity Models, Journal of Agricultural Economics 44:38-49. 
Byers, B. 1981. Periodic Points And Chaos For Expanding Maps Of The Interval, Bulletin of the Austrailian Mathematical Society 24: 79-83.

Carlson, J.B. 1987. Learning, Rationality, the Stability of Equilibrium and Macroeconomics, Economic Review, Federal Reserve bank of Cleveland.

Caswell, W.E. and J. A. Yorke 1986. Invisible errors in dimension calculations: geometric and systematic effects, in G. Mayer-Kress ed., Dimensions and Entropies of Chaotic Systems. Springer Series in Synergetics, Vol. 32 Berlin, Springer-Verlag.

Chan, K.S. and H. Tong 1986. On Tests For Nonlinearity In Time Series Analysis. Journal of Forecasting 5:217-218.

Chang, S. and J. Wright 1981. Transitions and Distributions Functions for Chaotic Systems, Physical Review A 23:1419-1433.

Chavas, J.P. and M.T. Holt 1991. On Nonlinear Dynamics: the Case of the Portk Cycle, Journal of the American Agricultural Economic Association, 73:820-828.

Cheung, Y.W. 1993. Tests for Fractional Integration: A Monte Carlo Investigation, Journal of Time Series Analysis, 14:331-345.

Chiarella, C. 1988. The Cobweb Model: Its Instability and the Onset of Chaos, Economic Modelling 4:377-384.

Cochrane, J.H. 1988. How Big Is The Random Walk In GNP? Journal of Political Economy 96:893-920.

Cochrane, J.H. 1991. A Critque Of The Application Of Unit Root Tests. Journal of Economic Dynamics and Control 15:275-284.

Collard, J.E. 1991. A B-P ANN Commodity Trader in Advances in Neural Information Processing Systems 3: Lippmann, R.P. Moody, J.E. and Touretzky, D.S. (eds.) San Mateo, CA: Morgan Kaufman Publishers.

Collet, P. and J.P. Eckmann 1980. Iterated Maps on the Interval as Dynamical Systems, Boston, MA:Birkhauser.

Conner, J.T., and R.D. Martin 1994. Recurrent Neural Networks and Robust Time Series Prediction, IEEE Transactions on Neural Networks 5:240-253.

Conover, W.J. 1980. Practical Nonparametric Statistics. New York: John Wiley \& Sons. 
Coppes, R.C. 1995. Are Exchange Rate Changes Nornally Distributed? Economic Letters 47:117-121.

Cotter, N.E. 1990. The Stone-Weierstrass Theorem and Its Application to Neural Networks, IEEE Transactions on Neural Networks 1:290-295.

Cromwell, J., Labys, W.C. and M. Terraza 1991. Testing Methods For The Econometric Analysis Of Time Series. Working Paper No. 151, Agricultural and Resource Economics Program, West Virginia University.

Cugno, F. and L. Montrucchio 1984. Some New Technigues for Modelling Nonlinear economic Fluctuations: A Brief Survey, in R.M. Goodwin, M. Kruger and A. Vercelli, eds. Nonlinear Models of Economic Growth, Lecture Notes in Economics and Mathematical Systems 228 Berlin:Springer-Verlag 146-165.

D'Agostino, R.B. and M.A. Stephens 1986. Goodness-of-fit techniques. New York: Marcel Dekker.

Daubechies, I., 1988. Orthonormal Bases Of Compactly Supported Wavelets, Communication of Pure and Applied Mathematics 41:909-996.

Daubechies, I. 1990. The Wavelet Transform, Time-Frequency Localization And Signal Analysis, IEEE transactions on Information Theory 36:961-1005.

Daubechies, I. 1992. Ten Lectures on Wavelets, Number 61 in CBMS_NSF Series in Applied Mathematics(SIAM, Philadelphia).

Day, R.H. 1982. Irregular Growth Cycles, American Economic Review 72:406-414.

Davidson, R., W.C. Labys, and J.B. Lesourd, Wavelet Analysis of Commodity Price Behavior, 1998. Research paper, Regional Research Insitute, West Virginia University, Morgantown, West Virginia.

Day, R.H. 1983. The Emergence Of Chaos From Classical Economic Growth, Quarterly Journal of Economics 98:201-213.

Deaton, A. and G. Laroque, 1992. On the Behavior of Commodity Prices, Review of Economic Studies, 59:1-23.

Deaton, A. and G. Laroque, 1995. Estimating Nonlinear Rational Expectations Commodity Price Models, Journal of Applied Econometrics, 10:510-540.

Decoster, G. and D. Mitchell 1988. Empirical Chaos Dectection Procedures: A Cookbook Approach, Working Paper, Department of Economics, West Virginia University. 
Decoster, G.P. Labys, W.C. and D.W. Mitchel 1992. Evidence of Chaos in Commodity Futures Prices, The Journal of Futures Markets, 12:291-305.

Devaney, R 1989. An Introduction to Chaotic Dynamical Systems New York:Addison Wesley.

Dickey, D.A. and W.A. Fuller 1979. Distribution Of The Estimators For Autoregressive Time Series With A Unit Root. Journal of the American Statistical Association 74:427-31.

Dickey, D.A. and W.A. Fuller 1981. Likelihood Ratio Tests For Autoregressive Time Series With A Unit Root. Econometrica 49:1057-1072.

Dickey, D.A., W.R. Bell and R.B. Miller 1986. Unit Roots In Time Series Models: Tests And Implications. American Statistician 40:12-26.

Dickey, D.A. and S.G. Pantula 1987. Determining the order of differencing in autoregressive processes. Journal of Business and Economic Statistics 5:455-62.

Diebold, F.X. and M. Nerlove 1989. Unit roots in economic time series: a selective survey. In Advances in Econometrics; Cointegration, Spurious Regressions, and Unit Roots. T.B. Fomby and G.F. Rhodes, eds., Greenwich, CT: Jai Press.

Diebold, F.X. and Rudebusch, G.D. 1989. Long Memory and Persistence in Aggregate Output, Journal of Monetary Economics 24:189-209.

Ding, Z. 1994. Time Series Analysis of Speculative Returns, Ph.D. dissertation, Department of Economics, University of California, San Diego.

Ding, Z. and C.W.J. Granger 1994. Modeling Volatility Persistence of Speculative Returns. A New Approach," UCSD Economics Department, Discussion Paper 94-05.

Donoho, D.L. 1992. De-Noising By Soft-Thresholding, Technical Report 409, Stanford University, Department of Statistics.

Donoho, D.L., I.M. Johnstone, G. Keryacharian, and D. Picard 1995. Wavlet Shrinkage: Asymptopia? Journal of the Royal Statistical Society, B. 57:301-369.

Dopfer, K. 1991. The Complexity of Economic Phenomena: Reply to Tinbergen and Beyond, Journal of Economic Issues 25:39-71.

Eckmann, J. and D. Ruelle 1985. Ergodic Theory of Chaos and Strange Attractors, Reviews of Modern Physics 57:617-656. 
Engle, R. 1982. Autoregressive Conditional Heteroskedasticity With Estimates Of The Variance Of The U.K. Inflation. Econometrica 50:987-1007.

Engle, R.F. and T. Bollerslev 1986. Modelling The Persistence Of Conditional Variances. Econometric Reviews 5 1:1-50.

Engle, R.F., D.M. Lilien and R.P. Robbins 1987. Estimating Time Varying Risk Premia In The Term Structure: The ARCH-M Model. Econometrica 55:391-408.

Evans, G.B.A. and N.E. Savin 1981. Testing for unit roots:1. Econometrica 49:753-779.

Ezekiel, M. 1938. The Cobweb Theorem, Quarterly Journal of Economics 53:255-280.

Fama, E. 1970. Efficient Capital Markets: A Review of Theoretical and Empirical Work, Journal of Finance. 15:383-417.

Fama, E. and Roll, R. 1968. Some Properties Of Symmetric Stable Distributions, Journal of the American Statistical Association. 63:817-36.

Famer, J.D. and J.J. Sidorowich 1987. Predicting Chaotic Time Series, Physical Review Letters 59:845-848.

Fang, H., K.S. Lai and M.Lai, 1994. Fractal Structure in Currency Futures Price Dynamics, The Journal of Futures Markets 14:169-181.

Frank, M. and Stengos, T. 1988. Chaotic Dynamics in Economic Time Series, Journal of Economic Surveys 2:103-133.

Frank, M. and Stengos, T. 1988. Some Evidence Concerning Macroeconomic Chaos, Journal of Monetary Economics, 22:423-438.

Frank, M. and Stengos, 1989. Measuring the Strangeness of Gold and Silver Rates of Return, Review of Economic Studies 56:553-567.

Franses, P.H. 1995. Testing for Seasonal Unit Roots in Monthly Data, Working Paper, Econometric Institute, Erasmus University, The Netherlands.

French, B.C. G.A. King, and D.D. Minami 1985. Planting and removal of perennial crops, American Joural of Agricultural Economics 53:478-90.

Fuller, W.A. 1976. An Introduction to Statistical Time Series. New York: John Wiley and Sons.

Fuller, W.A., D.P. Hasza and J.J. Goebel 1979. Estimation of the Parameters of Stochastic Difference Equations. Statistical Laboratory Report Iowa State University. 
Gabr, M.M. and T. Subba Rao 1981. The Estimation And Prediction Of Subset Bilinear Time Series Models With Applications. Journal of Time Series Analysis 2:155-71.

Gelb, A.H. 1979. A Spectral Analysis of Coffee Price Oscillations, International Economic Review 20:485-514.

Gerlow, M.E., S.H. Irwin and T. Liu, 1993. Economic Evaluation of Commodity Price Forecasting Models, International Journal of Forecasting 9:387-397.

Geweke, J.F. and Meese, R.A. 1981. Estimating Regression Models Of Finite But Unknown Order. International Economic Review 22:55-70.

Gilbert, C. 1990. The Rational Expectations Hypothesis in Models of Primary Commodity Prices, International Economics Department, The World Bank.

Gilbert, C. 1985. Efficient Market Commodity Price Dynamics, World Bank, Commodity Studies and Projections Division, Working Paper 1985-4.

Gilbert, C. and Palaskas 1990. Modeling Expectations Formation in Primary Commodity Markets in D. Sapsford and L. Winters (eds.) Primary Commodity Prices: Economic Models and Policy, Cambridge: Cambridge University Press.

Gleick, J. 1987. Chaos:Making a New Science. Viking Penguin: New York.

Godfrey, L.G. 1979. Testing The Adequacy Of A Time Series Model. Biometika 66:67-72.

Goffe, W.L. 1993. Wavelets In Macroeconomics: An Introduction, in D.A. Belsley(Ed.), Computational Economics and Econometrics, Volume 2. (Kluwer Academic Press, Amsterdam).

de Gooijer, J.G., B. Abraham, A. Gould and L. Robinson 1985. Methods For Determining The Order Of An Autoregressive-Moving Average Process: A Survey. International Statistical Review 53:301-29.

de Gooijer, J.G. and K. Kumar 1992. Some Recent Developments In Non-Linear Time Series Modeling, Testing And Forecasting. International Jounral of Forecasting 8:135-156.

Granger, C.W.J. 1981. Some Properties Of Time Series Data And Their Use In Econometric Model Specification. Journal of Econometrics 35:143-159.

Granger, C.W.J. 1991. Developments In The Nonlinear Analysis Of Economic Series. Scandanavian Journal of Economics 93:263-276. 
Granger, C.W.J. and Z.Ding 1993. Some Properties of Absolute Return: An Alternative Measure of Risk, USCD Economics Department, Discussion Paper 93-98.

Granger, C.W.J. and Z. Ding 1994. Varieties of Long Memory Models, UCSD Economics Department. Discussion paper.

Granger, C.W.J. and A.D. Hughes 1971. A New Look At Some Old Data: The Beveridge Wheat Price Series. Journal of the Royal Statistical Society A 134:413-28.

Granger, C.W.J. and P. Newbold 1974. Spurious Regressions In Econometrics. Journal of Econometrics 26:1045-1066.

Granger, C.W.J. and A.P. Andersen 1978. An Introduction To Bilinear Time Series Models. Gottingen: Vandenhoek and Ruprechet.

Granger, C.W.J. and P. Newbold 1986. Forecasting Economic Time Series. 2nd edition. Academic Pres, New York, NY.

Granger, C.W.J. and M. King and H. White 1992. Comments on Testing economic theories and the use of model selection criteria. Discussion paper 92-18, Department of Economics, University of California, San Diego.

Grassberger, P and I. Procaccia 198a. Measuring the Strangeness of Strange Attractors, Physica D 9:189-208.

Grassberger, P. and I. Procaccia 1983b. Estimation of the Kolmogorov Entropy from a Chaotic Signal, Physical Review 28:2591-2593

Greenblatt, S.A. Wavelets in Econometrics: An Application to Outlier Testing, Working Paper, Department of Economics, University of Reading, Reading, Berkshire, RG6 2AA, UK.

Greenword, D. 1991. An Overview of Neural Networks, Behavior Science 36:1-32.

Grudnitski, G. and L. Osburn 1993. Forecasting S\&P and Gold Futures Prices: An Application of Neural Networks, The Journal of Futures Markets 13:631-643.

Haggan, V., S.M. Heravi and M.B. Priestley 1984. A study of the application of state depedent models in non-linear time series analysis. Journal of Time Series Analysis 5:69-102.

Haggan,V. and T. Ozaki 1981. Modelling non-linear random vibrations using an amplitude-dependent autoregressive time series model. Biometrika 68:184-89. 
Haggan, V. S.M. Heravi and M.B. Priestley 1984. A Study Of The Application Of State Depedent Models In Non-Linear Time Series Analysis. Journal of Time Series Analysis 5:69-102.

Hannan, E.J. 1980. The Estimation Of The Order Of An ARMA Process. American Statistician 8:1071-81.

Hannan, E.J. 1982. A Note On Bilinear Time Series Models. Stochastic Process Applications 12:221-24.

Hannan, E.J. and B.G. Quinn 1979. The Determination Of The Order Of An Autoregression. Journal of the Royal Statistical Society Series B 41:190-195.

Hao, Bai-Lin 1984. Chaos. Singapore: World Scientific Publishing Co,

Hardle, W. 1990. Applied Nonparametric Regression ${ }_{2}$ Cambridge University Press.

Harvey, A.C. 1981. Time Series Models. Oxford:Philip Allan.

Hecht-Nielson R. 1990. Neurocomputing, Reading, MA: Addison-Wesley.

Hinich, M. 1982. Testing For Gaussinity And Linearity Of A Stationary Time Series. Journal of Time Series Analysis 3:169-176.

Holmes, P. 1979. A Non-linear Oscillator with a Strange Attractor, Communications in Mathematical Physics 50:69

Holmes, P. and R. Mannin 1988. Memory and Market Stability: The Case of the Cobweb, Economic Letters 28: 1-7.

Hosking, J.R., 1981. Fractional Differencing, Biometrika, 68:165-176.

Hosking, J.R. 1984. Modeling Persistence in Hydrological Time Series Using Fractional Differencing, Water Resources Research 20, 1898-1908.

Houthakker, H. 1961. Systematic and Random Elements in Short-term Price Movements, American Economic Review 51:164-72.

Howery, E.P. 1978. Comments On Estimating Structural Models Of Seasonality by R.F. Engle in A. Zellner, ed. Seasonal analysis of economic time series. U.S. Department of Commerce, Bureau of the Census, Washington, D.C. 298-302.

Hsieh, D.A. 1989. Testing For Nonlinear Dependence In Daily Foreign Exchange Rates. Journal of Business 62:339-368. 
Hsieh, D. A. 1988. The Statistical Properties of Daily Foreign Exchange Rates: 1974-1983. Journal of International Economics 24:129-145

Hsieh D. and B. LeBaron 1988. Finite Sample Properties of the BDS Statistic:Distribution Under the Null and Alternative Hypothesis, mimeo University of Chicago.

Hudson, M.A., Leuthold, R.M. and Sarassoro, G.F. 1987, Commodity Futures Pirce Changes: Recent Evidence for Wheat, Soybeans, and Live Cattle, The Journal of Futures Markets 7:287301.

Hylleberg, S., R.F. Engle, C.W.J. Granger and B.S.Yoo 1990. Seasonal Integration and Cointegration, Journal of Economtrics 44:215-238.

Jameson, M. 1983 Rational Expectations and U.S. Hog Cycle, in G. Rausser(ed), New Directions in Econometric Practice, Amsterdam, North Holland.

Jensen, M.J. 1994, Wavelet Analysis of Fractionally Integrated Processes, Working Paper, Department of Economics, Washington University, St.Louis MO 63130.

Jensen, R.V. and R. Urban 1984. Chaotic Price Behavior in the Nonlinear Cobweb Model, Economic Letters, 15:235-240.

Judge, G.G., W.E. Griffiths, R. Carter Hill, H. Lutkepohl, and T-C Lee 1985. The Theory and Practice of Econometrics New York: John Wiley and Sons.

Kaboudan, M.A. 1991 Chaotic Dynamics in Econometric Applications, Working paper, Mangement Science Department, Penn State University, Fogelsville, PA 18051.

Keenan, D.M. 1985. A Tukey Nonadditivity-Type Test For Time Series Non-Linearity. Biometrika 72:39-44.

Kendall, M.G. and M.A. Stuart 1961. The Advance Theory of Statistics. Volume 2, London: Griffin.

Kuan, C.M. and H. White 1991. Artificial Neural Networks: An Econometric Perspective Working paper, University of California, San Diego.

Kumar, K. 1986. On The Identification Of Some Bilinear Time Series Models. Journal of Time Series Analysis 7:117-22.

Kurths, J. and H. Hertzel 1987. An Attractor in a Solar Time Series, Physica D 25:165-172. 
Labys, W.C. 1973. Dynamic Commodity Models: Specification, Estimation And Simulation. Lexington, MA, Heath Lexington Books.

Labys, W.C. and C.W.J. Granger 1970. Speculation, Hedging And Commodity Price Forecasts. Lexington, MA: Heath Lexington.

Labys, W.C., Murcia, V. and M. Terraza 1991. Progress Econometriques et Series Temporelles. Working Paper, Center for Industrial Econometrics, University of Montpellier I.

Lapedes, A. and R. Farber 1987. Nonlinear signal processing using neural networks. Paper presented at the IEEE conference on neural information processing system-Natural and Synthetic.

Larson, A. 1960. Measurement of a Random Process in Future Prices, Food Research Institute Studies 1:313-324.

Lee, T.H., White, H. and C.W.J. Granger 1993. Testing For Neglected Nonlinearity In Time Series Models. Journal of Econometrics 56:289-90.

Leroy, S.F. 1989. Efficient Capital Markets and Martingales, Journal of Economic Literature 27: 4:1583-1621.

Leuthold, R. 1972. Random Walk and Price Trends: The Live Cattle Futures Market, Journal of Finance 27:879-889.

Li, W.K. 1984. On The Autocorrelation Structure And Identification. Journal of Time Series Analysis 5:173-82.

Li, T.Y. and J.A. Yorke 1975. Period Three Implies Chaos, American Mathematical Monthly, 82:985-992.

Liu, T., C.W.J. Granger, and W.P. Heller 1992. Using the Correlation Exponent to Decide If An Economic Series Is Chaotic, Working Paper, Department of Economics, University of California, San Diego.

Lippmann, R.P. 1987. An Introduction to Computing with Neural Nets. IEEE ASSP magazine $3: 4-22$.

Ljung, G.M. and Box, G.E.P. 1978. On A Measure Of The Lack Of Fit In Time Series Models. Biometrika 65:297-303.

Luukkonen, R., Saikkonen, P. and Terasvirta, T. 1988. Testing Linearity Against Smooth Transition Autoregressive Models. Biometrika 75,491-500. 
MacKuen, M., Erikson, R.S. and J.A. Stimson 1992. Moving Attractors And Character Of Partisan Change. American Political Science Review 86:100-110.

Mage, D.T. 1982. An Objective Graphical Method For Testing Normal Distributed Assumptions Using Probability Plots. American Statistician 36:116-120.

Mallat, S. 1989. A Theory for Multiresolution Signal Decomposition: The Wavelet Representation, IEEE Transactions on Information Theory, 38:617-643.

Mandelbrot, B. 1977a. Fractals: Form, Chance and Dimension, New York: Freeman.

Mandelbrot, B. 1977b. The Fractal Geometry of Nature, New York: Freeman.

Mandelbrot, B. and J. Van Ness, 1968. Fractional Browian Motions, Fractional Noises and Applications, SIAM Review 10:422-437.

Marvall, A. 1983. An Application Of Non-Linear Time Series Forecasting. Journal of Business and Economic Statistics 1:66-74.

May, R. 1976. Simple Mathematical Models with Very Complicated Dynamics, Nature 261:459-467.

McClave, J. 1975. Subset Autoregressive. Technometrics 17:213-220.

McClelland, J.L. and Rumelhart, D.E. 1988. Explorations in Parallel Distributed Processing. Cambridge, MA: MIT Press.

McKinnon, 1990. Critical Values For Co-Integration Tests. Working Paper, University of California, San Diego.

McLeary, R. and R.Hay 1980. Applied Time Series Analysis. Beverly Hills: Sage Publications.

McLeod, A.I. and W.K. Li 1983. Diagnostic checking ARMA time series models using squaredresiduals autocorrelations. Journal of Time Series Analysis 4:269-73.

Meyer, Y. 1990. Ondelettes et Operateurs, Vol. 1 and Vol. II, Paris, Hermann.

Meyer, Y. 1992. Wavelets and Operators. Cambridge, Cambridge University Press.

MicroTSP 7.0 1992. Quantitative Micro Software. Irvine, CA.

Mills, T.C. 1990. Time Series Techniques For Economists. Cambridge: Cambridge University Press. 
Mulhern, F.J. and R.J. Caprara 1994. A Nearest Neighbor Model for Forecasting Market Response, International Journal of Forecasting 10:191-207.

Muth, J.F. 1961. Rational Expectations and the Theory of Price Movements, Econometrica 29.315-55.

Nerlove, M.. 1958. Adaptive Expectatations and Cobweb Phenomena, Quarterly Journal of Economics. 23:

Nerrand, O. Roussel-Ragot, P., D.Urbani, L. Personnaz and G.Dreyfus, 1994. Training Recurrent Neural Networks: Why and How? An Illustration in Dynamical Process Modeling, IEEE Transactions on Neural Networks 5:178-184.

Newberry, D.M.G. and J.E. Stiglitz 1981. The Theory of Commodity Price Stabilization: A Study in the Economics of Risk, Oxford: Clarendon Press.

Nusse, H.E. and C.H. Hommes, 1990. Resolution of Chaos with Application to a Modfied Samuleson Model, Journal of Economic Dynamics and Control 14:1-19.

Osborne, A. and A. Provenzale 1989. Finite Correlation Dimension for Stochastic Systems with Power-Law Spectra, Physica D 35:357-381.

Ozaki, T. 1980. Non-Linear Time Series Models for Non-Linear Random Vibrations. Journal of Applied Probability 17:84-93.

Palaskas, T. and P. Varangas 1991. Is there Excess Co-Movement of Primary Commodity Prices? Working Paper, International Economic Department, The World Bank.

Panas, E. 1990. The Behavior of Athens Stock Prices. Applied Economics 22:1715-1727.

Parzen, E. 1974. Some Recent Advances In Time Series Modeling. IEEE Transactions on Automatic Control AC-19:723-30.

Pearson, E.S. and H.O. Hartley 1970. Biometrika Tables For Statisticians. Cambridge:Cambridge University Press.

Petruccelli, J.D. and N. Davies 1986. A Portmanteau Test For Self Exciting Threshold Autoregressive-Type Non-Linearity In Time Series. Biometrika 73:687-694.

Phillips, P.C.B. 1987. Time Series With A Unit Root. Econometrica 55:277-301.

Phillips, P.C.B. and P.Perron 1986. Testing For a Unit Root In Time Series Regressions. DP No. 781. Cowles Foundation, Yale University, New Haven. 
Phillips, P.C.B. 1988. Testing For A Unit Root In Time Series Regression. Biometrika 75:335346.

Phillips, P.C.B. 1986. Understanding Spurious Regressions In Econometrics. Journal of Econometrics 33:311-34.

Priestly, M.B. 1981. Spectral Analysis And Time Series. California: Academic Press, pp. 370-80.

Priestley, M.B. 1980. State-dependent models: a general apporach to non-linear time series analysis. Journal of Time Series Analysis 1:47-71.

Priestley, M.B. 1978. Nonlinear Models In Time Series Analysis. The Statistican 27:159-76.

Priestly, M.B. 1988. Non-Linear And Non-Stationary Time Series Analysis. Academic Press: New York.

Quinn, B.G. 1982. Stationarity And Invertibility Of Bilinear Models. Stochastic Process Applications 12:225-30.

Ramsey, J.B. 1969. Tests For Specification Error In Classical Linear Least Squares Regression Analysis. Journal of the Royal Statistical Society B 31:250-271.

Ramsey, J. and H. Yaun 1989. Bias and Error Bars in Dimension Calculations and Their Evaluation in Simple Simple Models, Physics Letters A 134:287-297

Ramsey, J. B. C.L. Sayers and P. Rothman 1990. The Statistical Properties Of Dimension Calculations Using Small Data Sets: Some Economoc Applications, International Economic Review 31: 991-1020.

RATS 3.0. VAR Econometrics, Minneapolis, MN.

Rausser, G.C. and F. Cargill 1970. The Existence of Broiler Cycles: An Application of Spectral Analysis, Americal Journal of Agricultural Economics 32:109-121.

Robinson, P.M. 1983. Nonparametric Estimators for Time Series, Journal of Time Series Analysis 4:185-208.

Robinson, P.M. 1987. Asymptotically efficient estimation in the presence of heteroskedasticity of unknown form, Econometrica 55:875-891.

Said, S.E. and D.A. Dickey 1984. Testing for unit roots in autoregressive moving-average models with unknown order. Biometrika 71:599-607. 
Said, S.E. and D.A. Dickey 1985. Hypothesis testing in ARIMA (p,1,q) models. Journal of the American Statistical Association 80:369-74.

Saikkonen, P. and R. Luukkonen 1988. Lagrange multiplier tests for testing on-linearities in time series models. Scandinavian Journal of Statistics 15:55-68.

Samuelson, P. 1965. Proof that Properly Anticipated Prices Fluctuate Randomly Industrial Management Review 6:41-49.

Savit, R. 1988. When Random is Not Random: An Introduction to Chaos in Market Prices, Journal of Futures Markets 8:271-289.

Sayers, C. 1990. Chaos and the Business Cycle, In Saul Krasner, Ed. The Ubiquity of Chaos, Washington, The American Association for the Advancement of Science.

Scheinkman, J. and B. LeBaron 1989a. Nonlinear Dynamics and Stock Returns, Journal of Business 7:311-37

Scheinkman, J. and B. LeBaron 1989b. Nonlinear Dynamics and GNP Data, in Barnett, W., J. Geweke and K.Shell (eds.) Economic Complexity: Chaos, Sunspots, Bubbles and Nonlinearity Cambridge: Cambridge University Press p213-227.

Schwarz, G. 1978. Estimating the Dimension of a Model. Annals of Statistics 6:461-464.

Schwert, G.W. 1987. Effects On Model Specification On Tests For Unit Roots In Macroeconomic Data. Journal of Monetary Economics 20:73-103.

Sharda, R. and Patil, R.B. 1990. Neural Networks as Forecasting Experts: An Empirical Test, International Joint Conference on Neural Networks, 11:491-494.

SHAZAM. User's Reference Manual. Version 6.2. New York:McGraw-Hill.

Shibata, R. 1980. Asymptotically Efficient Selection Of The Order Of The Model For Estimating Parameters Of A Linear Process. The Annals of Statistics 8:147-164.

Silverman, B. W. 1986. Density Estimation for Statistics and Data Analysis, London, Chapman and Hall.

Sims, C. 1984. Martingale-Like Behavior of Prices and Interest Rates, Discussion Paper No. 205, Department of Economics, University of Minnesota, Minneapolis.

Stengos, T. and E. Panas 1992. Testing the Efficiency of the Athens Stock Exchange: Some Results from the Banking Sector, Empirical Economics 17:239-252. 
Stevenson R. and R. Baer 1970. Commodity Futures: Trends or Random Walk? Reprinted in Selected Writings on Futures Markets, Peck, A. (ed.) Chicago Board of Trade, pp279-294

Stimson, J.A. 1991. Public Opinion In America: Moods, Cycles And Swings. Boulder: Westview Press.

Stutzer, M.J. 1980. Chaotic Dynamics and Bifurcation in a Macro Model, Journal of Economic Dynamics and Control, 2:353-376..

Subba Rao, T. 1981. A Cumulative Sum Test For Detecting Change In Time Series. International Journal of Control 34:285-93.

Subba Rao, T. and M.M. Gabr 1980. A Test For Linearity Of Stationary Time Series. Journal of Time series analysis 1:145-148.

Subba Rao, T. and M.M. Gabr 1984. An Introduction To Bispectral Analysis And Bilinear Time Series Models. Berlin: Springer-Verlag.

Subba Rao, T. 1981. On The Theory Of Bilinear Time Series Models. Journal of Royal Statistical Society B 43:244-55.

Subba Rao, T. and M.M. Gabr 1984. An Introduction To Bispectral Analysis And Bilinear Time Series Models. Lecture notes in statistics Vol. 24. Berlin: Springer-Verlag.

Takens, F. 1980. Detecting Strange Attractors in Turbulence, in D. Rand and B.S. Yound. Eds. Dynamical Systems and Turbulence. Lecture Notes in Mathematics 898, Berlin: Springer Verlag.

Taylor, S. 1968. Modelling Financial Time Series, New York: John Wiley \& Sons.

Tomek, W.G. 1994. Dependence in Commodity Prices: A Comment, The Journal of Futures Markets 14:103-109.

Tomek, W.G. and K.L. Robinson 1990. Agricultural Product Prices: London: Cornell University Press.

Tomek, W.G. and Myers, R.J. 1993. Empirical Analysis of Agricultural Commodity Prices: A Viewpoint, Review of Agricultural Economics 15:181-202.

Tong,H. 1983. Threshold Models In Non-Linear Time Series Analysis. Berlin: Springer-Verlag.

Tong, H. and K.S. Lim 1980. Threshold Autoregression, Limit Cycles And Cyclical Data. Journal of the Royal Statistical Society Series B 42:245-92. 
Tong, H. 1979. A Note On Local Equivalence Of Two Recent Approaches To Autoregressive Order Determination. Int. J. Control 29:441-446.

Tsay, R.S. 1988. Outliers, Level Shifts, And Variance Changes In Time Series. Journal of Forecasting 7:1-20.

Tsay, R.S. 1986a. Time Series Model Specification In The Presence Of Outliers. Journal of the American Statistical Association 81:132-41.

Tsay, R.S. 1986b. Nonlinearity test for Time series. Biometrika 73:461-466.

Tsay, R.S. 1987. Conditional Heteroskedastic Time Series Models. Journal of the American Statistical Association 82:590-604.

Tuan, P. and T.T. Lanh 1981. On the First Order Bilinear Time Series Model. Journal of Applied Probability 18:617-27.

Turnovsky, S.J. 1985. Stochastic Stability Of Short-Run Market Equilibrium Under Variations In Supply. Quarterly Journal of Economics .666-681.

Ullah, A. 1988. Nonparametric Estimation of Econometric Functionals, Canadian Journal of Economics, 21:625-658.

Wallis, K.F. 1987. Time Series Analysis of Bounded Economic Variables. Journal of Time Series Analysis 8:115-23.

Weiss, A.A. 1984. ARMA models with ARCH errors. Journal of Time Series Analysis 5:129-43.

White, H. 1998. Economic Prediction using Neural Networks: The Case of IBM Daily Stock Returns, IEEE International Conference on Neural Networks, 11:451-458.

White, H. 1991. Parametric Statistical Estimation with Artificial Neural Networks, Department of Economics and Institute for Neural Computation, University of California, San Diego.

Williams, J.C. and Wright, B.D. 1991. Storage and Commodity Markets, Cambridge University Press.

Wolf, A, J. Swift, H. Swinney, and J. Vastano 1985. Determing Lyapunov Exponents from a Time Series, Physica D 40:285-317

Working, H. 1958. A Theory Of Anticipatory Prices American Economic Review 48:188-199.

Working, H. 1949. The Theory of the Price of Storage, American Economic Review 39:12541262. 
Working, H. 1934. A Random Difference Series for Use in the Analysis of Time Series, Journal of the American Statistical Association 9:11-24.

Wornell, G. 1993. Wavelet-Based Representation for the 1/f family of Fractal Processes, Proceeding of the IEEE, 81, 1428-1450.

Wornell, G. and A. Oppenheim 1992. Estimation of Fractal Signals from Noisy Measurments Using Wavelets, IEEE Transactions on Signal Processing, 40:611-623.

Wright, J. 1984. Method for Calculating Lyapunov Exponents, Physical Review A 29:2924-27

Wright, B. and J. Williams 1984. The Welfare Effects of the Introduction of Storage Quarterly Journal of Econmics 98:169-92.

Wright, B. and J. Williams 1982. The Economic Role of Commodity Storage Economic Journal 92:596-614.

Yang, S.R. and B.W. Brorsen, 1992, Nonlinear dynamics of Daily Cash Prices, American Journal of Agricultural Economics, 706-715. 


\section{APPENDIX A DATA DESCRIPTION AND SOURCES}

\section{BANANAS}

Central America, fresh f.o.b. Atlantic and Pacific Ports (International Monetary Fund), 1960.011994.06.

\section{BEEF}

All origin imported frozen boneless f.o.b. (International Monetary Fund). 1960.01-1994.06.

\section{COFFEE}

Average of daily prices (secretariat of the International Coffee Organization, London). Robustas, weighted average of ex-dock New York (60 percent), Angola Ambriz 2 BB, Uganda standard, 1960.01-1994.06.

\section{SOYBEANS}

U.S. Yellow No. 1, Average cash price, Chicago (USDA, Washington, DC), 1960.01-1994.06.

\section{WHEAT}

U.S. No. 2, Hard Red winter (ordinary), f.o.b. Gulf (International Wheat Council), 1960.011994.06.

\section{WOOL}

UK64's (dry-combed basis) (New Zealand Wool Marketing Corporation, Clacton-on-Sea, United Kingdom), 1960.01-1994.06. 


\section{APPENDIX B TEST PROCEDURES}

\section{(1) Ljung-Box and Box-Pierce Test}

\section{Test Procedure}

Step 1. Select the number of lags $k$ for the estimation of the sample autocorrelation coefficients $\rho$. Keep in mind that everything relies on the parameter $\mathrm{k}$, and that $\mathrm{k}$ is somewhat ambiquious.

Step 2. Given the number of lags, test the null hypothesis which implies linear independence in $\mathrm{x}(\mathrm{t})$

H0: $\rho(1)=\rho(2)=\ldots=\rho(k)=0$

HA: at least one $\rho(\mathrm{k})$ is non-zero

Compute the test statistic, $\mathrm{Q}_{\mathrm{i}}(\mathrm{k})$

i) $\mathrm{Q}_{1}(\mathrm{k})=\mathrm{T}(\mathrm{T}+2) \Sigma_{\mathrm{m}}(\mathrm{T}-\mathrm{m})^{-1} \rho^{2}(\mathrm{~m})$ Ljung-Box

ii) $\mathrm{Q}_{2}(\mathrm{k})=\mathrm{T} \Sigma_{\mathrm{m}} \rho^{2}(\mathrm{~m}) \quad$ Box-Pierce

Step 3. Choose the significance level $\alpha$ and the critical values of $\tau$, where the latter is distributed as chi-square with $\mathrm{k}$ degrees of freedom, $\chi^{2}(\mathrm{k})$.

Step 4. Reject the null hypothesis of linear independence if

$$
\mathrm{Q}_{\mathrm{i}}(\mathrm{k})>\tau
$$

\section{(2) Brock, Dechert and Sheinkman (BDS) Test}

\section{Test Procedure}

Step 1. Transform $x(t)$ to the unit interval, $[0,1]$.

Step 2. Choose a grid of values for $\varepsilon_{\mathrm{i}}=0.9^{\mathrm{i}}$ and $\mathrm{m}=\mathrm{i}+1$. 
Step 3. Again the null hypothesis reflects independence

$\mathrm{H} 0: \mathrm{x}(\mathrm{t})$ is independent

HA: $x(t)$ is dependent

The test statistic $\mathrm{W}$ provides the basis for the hypothesis test

$$
\mathrm{W}(\varepsilon, \mathrm{m}, \mathrm{T})=(\mathrm{T}-\mathrm{m}+1)^{1 / 2}\left[\mathrm{C}(\varepsilon, \mathrm{m}, \mathrm{T})-\left(\mathrm{C}(\varepsilon, 1, \mathrm{~T})^{\mathrm{m}}\right] / \mathrm{V}^{1 / 2}\right.
$$

Step 4. For the given significance level, $\alpha$, the critical values to test $\mathrm{H} 0$ are based on the number of observations dividing the selected embedding dimension.

i) If $\mathrm{T} / \mathrm{m}>200$, use the standard normal distribution, $\mathrm{N}(0,1)$, for the critical value, $\tau$.

ii) If $\mathrm{T} / \mathrm{m}<200$, use $\tau$ from tables in Brock, Hsieh and Lebarron (1991).

Step 5. Reject the null hypothesis of independence if $|W|>|\tau|$

\section{TESTING FOR LINEAR OR NONLINEAR DEPENDENCE}

\section{(3) Keenan Test}

\section{Test Procedure}

Step 1. Fit the model under the null hypothesis and calculate the fitted values $\mathrm{x}(\mathrm{t})$, the residuals, $\mathrm{e}(\mathrm{t})$, and the residual sum of squares $\mathrm{SSE}_{\mathrm{e}}=\Sigma \mathrm{e}^{2}(\mathrm{t})$.

$\mathrm{H} 0: \mathrm{x}(\mathrm{t})$ is an $\mathrm{ARMA}(\mathrm{p}, \mathrm{q})$ process

HA: $x(t)$ is not an $\operatorname{ARMA}(p, q)$ process

Step 2. From these fitted values, perform the regression of

$$
\begin{aligned}
& x^{2}(t) \\
& x^{2}(t)=\alpha_{0}+\Sigma_{i} \alpha_{i} x(t-i)+v(t) \quad \text { for } i=1, \ldots, m
\end{aligned}
$$

The selection of $\mathrm{m}$ is suggested not to be an important 
issue.

Step 3. Calculate the residuals, $v(t)$, and the residual sum of squares, $\mathrm{SEE}_{\mathrm{v}}$

Step 4. Perform the following regression to obtain the value of

$ß$. The residuals under the null hypothesis become

$\mathrm{e}(\mathrm{t})=\beta \mathrm{v}(\mathrm{t})+\mathrm{w}(\mathrm{t})$

Step 5. The residuals e(t) (i.e. residuals under the null) and $v(t)$ are reflected in the null and alternative hypothesis of step (1). Test the null hypothesis according to the statistic

$$
\mathrm{F}=\frac{\beta^{2}\left(\mathrm{SSE}_{\mathrm{v}}\right)(\mathrm{T}-2 \mathrm{~m}-2)}{\left(\left(\mathrm{SSE}_{\mathrm{e}}\right)-\left(\AA^{2} \mathrm{SSE}_{\mathrm{v}}\right)\right)}
$$

Step 6. For a given significance level, $\alpha$, obtain the critical value to compare to $\mathrm{F}$ from the table of the $\mathrm{F}$ distribution with 1 and T-2m-2 degrees of freedom, $\mathrm{F}(1, \mathrm{~T}-2 \mathrm{~m}-2)$.

Step 7. Reject the null hypothesis and assume nonlinear dependence if $F>\tau$.

\section{(4) McLeod-Li Test}

\section{Test Procedure}

Step 1. Select lag length $\mathrm{k}$ based on the sample frquency and estimate the autocorrelation function of $\mathrm{x}^{2}(\mathrm{t})$.

$$
\rho_{\mathrm{x}}{ }^{\mathrm{x}}(\mathrm{k})=\frac{\underline{\Sigma}_{\mathrm{t}}\left(\mathrm{x}^{2}(\mathrm{t})-\sigma^{2}\right)\left(\mathrm{x}^{2}(\mathrm{t}+\mathrm{k})-\sigma^{2}\right)}{\Sigma_{\mathrm{t}}\left(\mathrm{x}^{2}(\mathrm{t})-\sigma^{2}\right)^{2}}
$$

where $\sigma^{2}$ is the variance of $x^{2}(t)$.

Step 2. The null hypothesis now reflects independence 
$\mathrm{H} 0: \mathrm{x}^{2}(\mathrm{t})$ is independent

HA: $x^{2}(t)$ is dependent

The same hypothesis and testing procedure can be followed for $|x(t)|$. Compute the following statistic to test the hypothesis,

$\mathrm{Q}_{\mathrm{xx}}(\mathrm{k})=\mathrm{T}(\mathrm{T}+2)(\mathrm{T}-\mathrm{m})^{-1} \rho_{\mathrm{xx}}^{2}(\mathrm{~m})$

Step 3. For a given significance level, $\alpha$, obtain the critical value $\tau$ to compare to $\mathrm{Q}_{\mathrm{xx}}(\mathrm{k})$ from the table of the chi-square distribution, $\chi^{2}(\mathrm{k})$

Step 4. Reject the null hypothesis of linear dependence if $\mathrm{Q}_{\mathrm{xx}}(\mathrm{k})>\tau$.

\section{(5) Hsieh Test}

\section{Test Procedure}

Step 1. Fit an $A R(p)$ model to the data to remove the linearity. Estimate the third order moment of the residuals e(t)

$$
\rho_{\text {eee }}(\mathrm{i}, \mathrm{j})=\left[(1 / \mathrm{T}) \Sigma_{\mathrm{t}} \mathrm{e}(\mathrm{t}) \mathrm{e}(\mathrm{t}-\mathrm{i}) \mathrm{e}(\mathrm{t}-\mathrm{j})\right] /\left[(1 / \mathrm{T}) \Sigma \mathrm{e}^{2}(\mathrm{t})\right]^{1.5}
$$

If a linear specification does not exist for $\mathrm{x}(\mathrm{t})$, the residuals $\mathrm{e}(\mathrm{t})$ in the experessions can be replaced by the variable $\mathrm{x}(\mathrm{t})$.

Step 2. Formulate the following null and alternative hypotheses

$\mathrm{H} 0: \mathrm{x}(\mathrm{t})$ possess a mulitplicative or variance nonlinearity

HA: $\mathrm{x}(\mathrm{t})$ possess an additive or mean nonlinearity

Step 3. In order to test the null hypothesis, compute the following test statistic

$$
V(i, j)=T^{1 / 2} \rho_{\text {eee }}(i, j) /\left[w(i, j) / \sigma_{e}{ }^{6}\right]
$$

where $w(i, j) / \sigma_{e}{ }^{6}$ can be consistently estimated by 


$$
\left[(1 / \mathrm{T}) \Sigma \mathrm{e}^{2}(\mathrm{t}) \mathrm{e}^{2}(\mathrm{t}-\mathrm{i}) \mathrm{e}^{2}(\mathrm{t}-\mathrm{j})\right] /\left[\Sigma \mathrm{e}^{2}(\mathrm{t}) / \mathrm{T}\right]^{3}
$$

Step 4. For a selected significance level $\alpha$, find the critical value $\tau$ for testing the null hypothesis of multiplicative nonlinearity using the standard normal distribution, $\mathrm{N}(0,1)$.

Step 5. Reject the null hypothesis, if $|\mathrm{V}(\mathrm{i}, \mathrm{j})|>|\tau|$

\section{(6) ARCH Test}

\section{Test Procedure}

Step 1. Select the time series model of interest for $x(t)$ and perform the regression and calculate the residuals $\mathrm{e}(\mathrm{t})$. Then perform the second regression of the following form for a selected value of $\mathrm{p}$.

$$
\mathrm{e}^{2}(\mathrm{t})=\alpha_{0}+\Sigma_{\mathrm{i}} \alpha_{\mathrm{i}} \mathrm{e}^{2}(\mathrm{t}-\mathrm{i})+\mathrm{v}(\mathrm{t}) \text { for } \mathrm{i}=1, \ldots, \mathrm{p}
$$

Step 2. Establish the null hypothesis of no ARCH effect.

H0: No ARCH effect

HA: ARCH effect present

In order to test the null hypothesis, the following test statistic is used

$$
\mathrm{LM}=\mathrm{TR}^{2}
$$

where $\mathrm{T}$ is the number of observations and $\mathrm{R}^{2}$ is the coefficient of determination of the regression conducted in step 1.

Step 3. For the given significance level, $\alpha$, the critical value $\tau$ is based on chi-square distribution with $\mathrm{p}$ degrees of freedom, $X^{2}(\mathrm{p})$. 